\title{
An Educational Intervention for Skin Cancer Prevention with Hospital Staff: A Pilot Project for System Change
}

\author{
Amy Fran Bruce \\ West Virginia Univerisity, afshaw@mix.wvu.edu
}

Follow this and additional works at: https://researchrepository.wvu.edu/etd

Part of the Occupational and Environmental Health Nursing Commons, Psychiatric and Mental Health Nursing Commons, and the Public Health and Community Nursing Commons

\section{Recommended Citation}

Bruce, Amy Fran, "An Educational Intervention for Skin Cancer Prevention with Hospital Staff: A Pilot Project for System Change" (2019). Graduate Theses, Dissertations, and Problem Reports. 3911. https://researchrepository.wvu.edu/etd/3911

This Problem/Project Report is protected by copyright and/or related rights. It has been brought to you by the The Research Repository @WVU with permission from the rights-holder(s). You are free to use this Problem/Project Report in any way that is permitted by the copyright and related rights legislation that applies to your use. For other uses you must obtain permission from the rights-holder(s) directly, unless additional rights are indicated by a Creative Commons license in the record and/ or on the work itself. This Problem/Project Report has been accepted for inclusion in WVU Graduate Theses, Dissertations, and Problem Reports collection by an authorized administrator of The Research Repository @ WVU. For more information, please contact researchrepository@mail.wvu.edu. 
An Educational Intervention for Skin Cancer Prevention with Hospital Staff:

A Pilot Project for System Change

Amy F. Bruce, MSN, RN, NE-BC

Doctoral Capstone Project submitted

to the West Virginia University School of Nursing

in partial fulfillment of the requirements for the

Doctor of Nursing Practice Degree

Theresa Cowan, DHED, MSN, RN, ACNS-BC, Chair

Cynthia Armstrong Persily, PhD, RN, FAAN

Georgia Daniel, APRN, RN, DCNP

Kari Sand-Jecklin, EdD, MSN, RN, AHN-BC

Department of Nursing

Morgantown, West Virginia

2019

Keywords: Skin Cancer, Prevention, Educational Intervention, System Change

Copyright 2019 Amy F. Bruce, MSN, RN, NE-BC 


\begin{abstract}
An Educational Intervention for Skin Cancer Prevention with Hospital Staff:

A Pilot Project for System Change
\end{abstract}

Amy F. Bruce, MSN, RN, NE-BC

PURPOSE: The purpose of this project was to design, implement, and evaluate an educational intervention provided to health care professionals using the Sun Smart U Curriculum created by the Skin Cancer Foundation (2017).

METHODS: Using a pretest-posttest design with a convenience sample $(\mathrm{N}=40)$, this intervention targeted the health professionals and staff from a residential/acute care facility caring for pre-adolescent, adolescent, and adult populations. Aims of this project included: (1) the staff's baseline level of knowledge regarding sun protective behaviors using the Sun Smart U Curriculum, (2) the staff's level of knowledge regarding sun protective behaviors post educational intervention using the Sun Smart U Curriculum, (3) the staff's intent to change behavior; and, (4) the feasibility of implementing behavior change within the organization.

RESULTS: Parametric summary statistics were used to report pretest-posttest survey data. The Continuing Professional Development-Reaction (CPD-R) tool (Légaré et al., 2017) was used to measure intent to change sun protective behaviors. The CPD-R is reported as a valid and reliable tool (Cronbach's coefficients for constructs varied from 0.77 to 0.85 ) for assessing the impact of $\mathrm{CPD}$ activities on the behavioral intentions of healthcare providers. Results from this project indicate a significant increase in test scores from pretest to posttest knowledge $(95 \% \mathrm{CI}$, Cohen's $\mathrm{d}=0.463, \mathrm{p}=0.006$ ). The majority of participants would encourage the children to use sunscreen at the facility $(100 \%)$, and $98 \%$ indicated the organization would support the behavior change; however, $35 \%$ indicated likeliness to personally use sunscreen and/or sun prevention methods at the facility. Participants with a higher level of education were significantly more likely to engage in sun protective behaviors $\left(\mathrm{x}^{2}=35.50, d f=20, \mathrm{p}=0.018\right)$.

CONCLUSIONS: In summary, this study addressed two important necessities in skin cancer prevention: the need for a staff intervention to improve knowledge regarding sun safe behaviors, and an educational intervention targeting health care professionals' intent to change behaviors.

IMPLICATIONS: A pre-determined curriculum was used to compare hospital staff's baseline level of knowledge to posttest results regarding sun protective behaviors after an educational intervention. Although staff indicated a significantly higher level of knowledge post intervention, future dissemination of research would recommend a 3 to 6-month follow-up evaluating actual changes in behavior. Those individuals with a higher level of education indicated a likeliness to engage in sun protective behavior. This may imply a further need for targeting those individuals with lower education and socioeconomic status to encourage skin screening and sun protective behavior changes resulting in lower morbidity and mortality of melanoma skin cancer. Health care professionals would benefit from specific sun protective education to improve both personal health and patient care practices. 


\section{Acknowledgements}

The concept of graduate education was never an option for me. I had always known I would push myself to the very limits of existence from an early age, even to my own detriment. Being an only child propelled this inner desire to compete with no one, barring myself. Hence, I view the completed work before me not only as an accomplishment of scholarship and professional viability, but as a completed goal once thought unattainable. I was not considered a child of privilege, and therefore was not afforded the luxuries or opportunities most children have - my road was self-paved against the grain of a tranquil childhood.

A very special thank you to my committee members for their unrelenting support, guidance, and enthusiasm during the phases of this project. To Dr. Theresa Cowan, committee chairperson, who guided me through every phase of the process with encouragement and confidence. Also, with heartfelt recognition and gratitude, I thank Dr. Cynthia Armstrong Persily for her wisdom, leadership, and networking abilities connecting me with those individuals who continue to help propel my career forward. Thank you to Georgia Daniel, my content expert, who contributed the evidence-based practice knowledge to elevate the importance of my work. Lastly, Dr. Kari Sand-Jecklin, a scholar whose energy and vision for this project kept me persevering to completion.

I would like to recognize other good friends and colleagues that I have called upon for assistance during the development of this project. Dr. Pamela L. Alderman and Dr. Evelyn Klocke who served as my mentors during the final phases of completion, and Dr. Aida Jimenez, who spent countless hours assisting me with the statistical significance and interpretation of my data - I have a great respect for your abilities. Thank you, Melinda Stoecklin, for your emotional support from beginning to end, and those most important parts in between. 
This journey has not come without its own trials and tribulations. There are those individuals that have sacrificed much more than I during my professional evolution. My husband Ivan exhibits the strength, support, and love I needed to continue this goal against all odds - you are my rock. My 3 most precious, Olivia, Roman, and IJ, have inspired me to become not only a better mother, but a successful female role-model serving as the foundation for their future growth and development. For my 5 step-children, Joshua, Lindsey, Ellen, Caroline, and Alex, who helped me learn to expand my horizons and love larger. Lastly, for my mother who helped instill the determination of self-reliance, and my grandmother for all the much-needed prayers.

That being said, this entire work is dedicated to my late grandfather, Newell Lee Haynes, who will never see me walk across the stage for my terminal degree but is watching me from Heaven - I love you and thank you for being my 'Dad'. I told you I would do it, and I have never broken a promise. 


\section{Table of Contents}

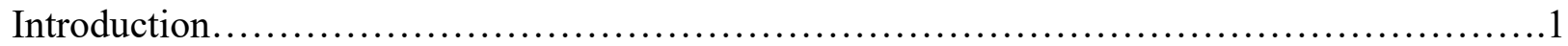

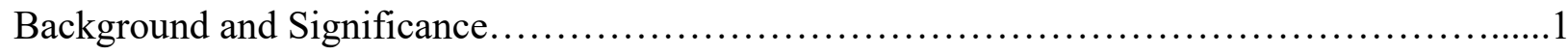

Problem Statement and Project Purpose..........................................5

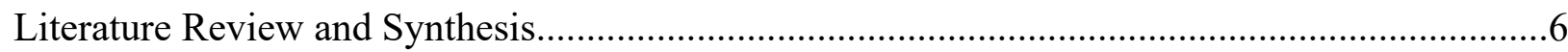

Synthesis and Conclusion...............................................20

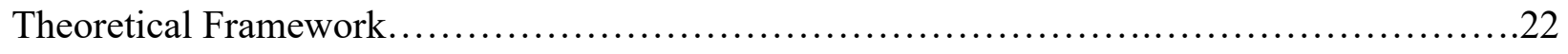

Project Description...............................................................27

Evidence-Based Design and Intervention Plan................................27

Theoretical Framework Support of Project.......................................29

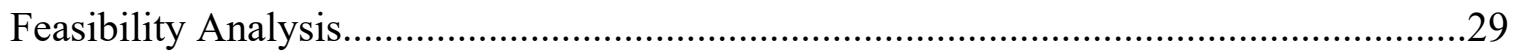

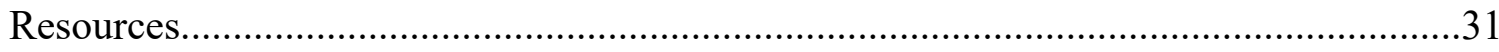

Congruence of Organization's Strategic Plan to Project Timeline...................................32

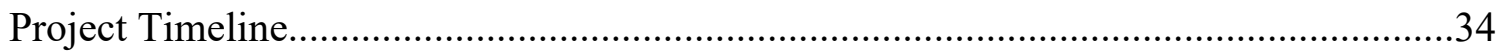

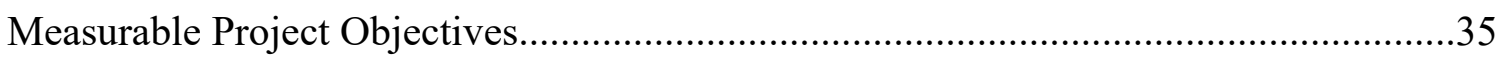

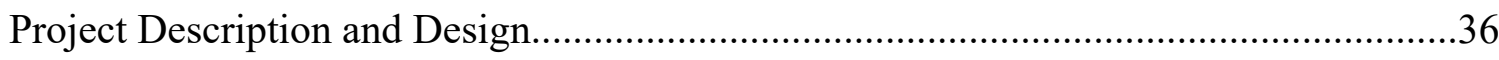

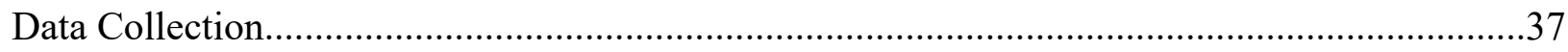

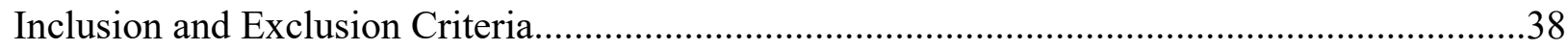

Project Evaluation Plan and Measurement Instruments......................................................38

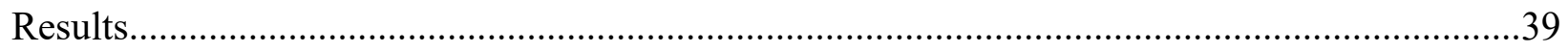

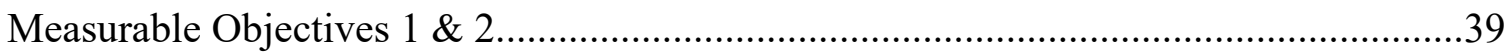

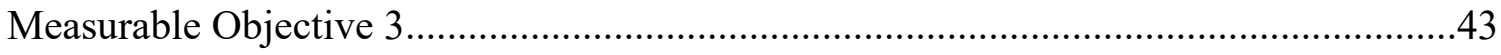

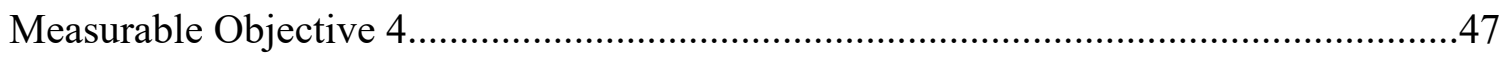




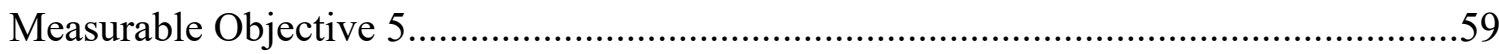

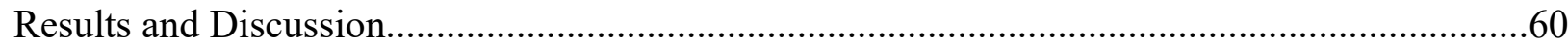

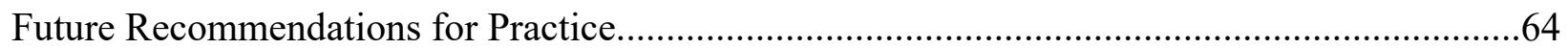

Evaluation of Theoretical Framework Integration..................................................65

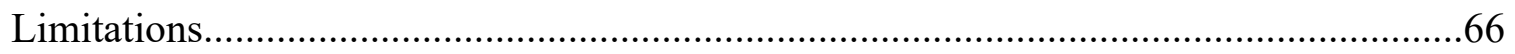

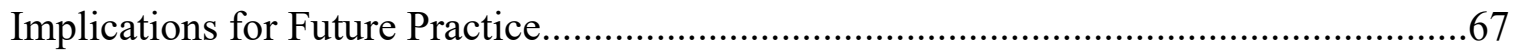

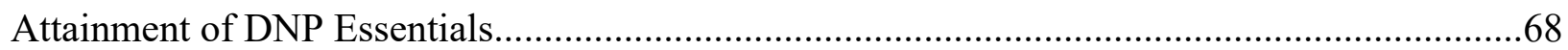

Essential I: Scientific Underpinnings for Practice...................................................69

Essential II: Organizational and Systems Leadership for Quality Improvement and Systems Thinking

Essential III: Clinical Scholarship and Analytical Methods for Evidence-Based

Practice. .70

Essential IV: Information Systems/Technology and Patient Care Technology for the Improvement and Transformation of Health Care..................................70

Essential V: Health Care Policy for Advocacy in Health Care.....................................71

Essential VI: Interprofessional Collaboration for Improving Patient and Population

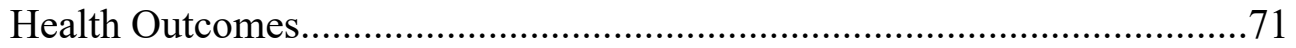

Essential VII: Clinical Prevention and Population Health for Improving the Nation's

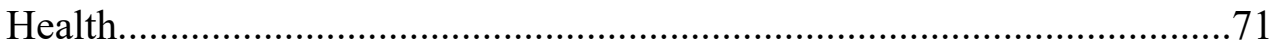

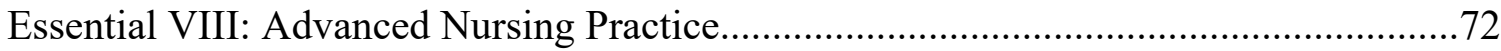

Summary

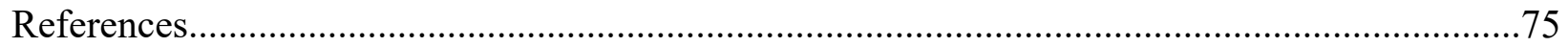

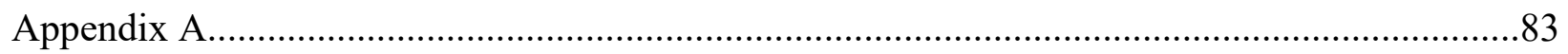

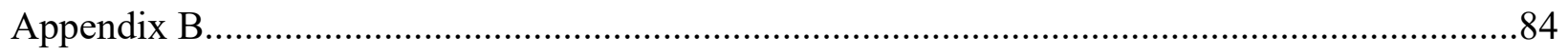




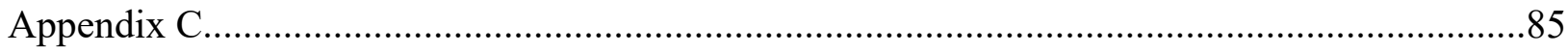

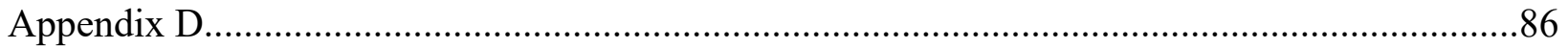

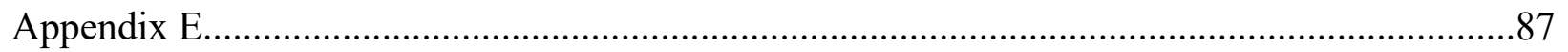

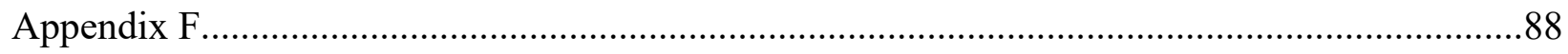

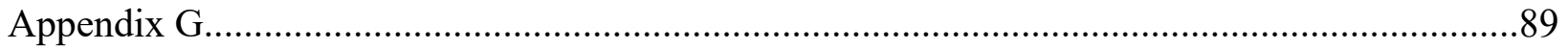

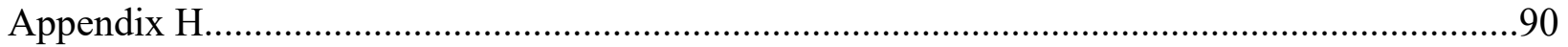

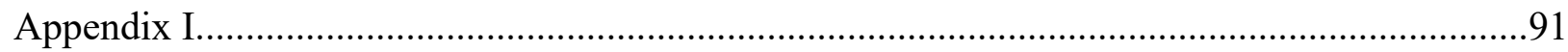

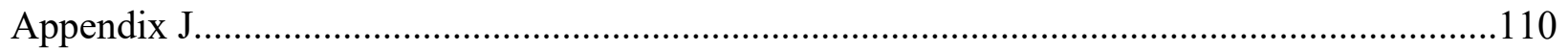

\section{Index of Tables}

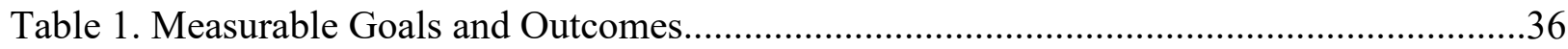

Table 2. Descriptive Statistics Pretest and Posttest and Percentage Change on Exam Scores......40

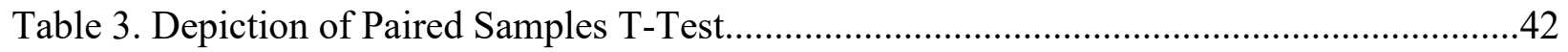

Table 4. Descriptive Statistics for Sun Exposure..................................................................

Table 5. Contingency Table Testing Association Between Age ad Sun Protection Usage............52

Table 6. Contingency Table Testing Association Between Gender and Sun Protection Usage

Table 7. Contingency Table Testing Association Between Level of Education and Sun

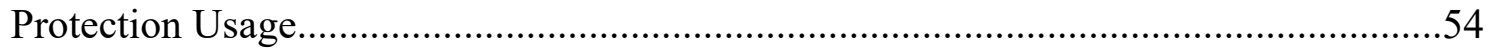

Table 8. Contingency Table Testing Association Between Sun Protection Usage and

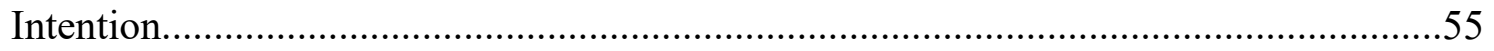

Table 9. Contingency Table Testing Association Between Sun Protection Usage and

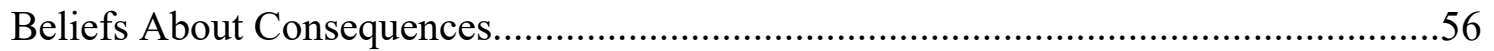

Table 10. Contingency Table Testing Association Between Sun Protection Usage and Social Influence. 
Table 11. Contingency Table Testing Association Between Sun Protection Usage and Beliefs About Capabilities .58

Table 12. Contingency Table Testing Association Between Sun Protection Usage and Moral Norms

\section{Index of Figures}

Figure 1. Distribution of the differences between the mean pretest and posttest scores.

Figure 2. Summary of CPD-Reaction questionnaire scores on items and 5-constructs.

Figure 3. Average CPD-Reaction scores for all participants on each construct.......................46

Figure 4. Spider plot of all 5 constructs measured by the CPD-R tool questionnaire................46

Figure 5. Patient demographic information displaying gender.......................................4

Figure 6. Patient demographic information displaying educational level..............................48

Figure 7. Patient demographic information displaying preferred sun protection......................49

Figure 8. Patient demographic information illustrating age at time of survey.........................50

Figure 9. Personal Practice Change survey results........................................................60 
An Educational Intervention for Skin Cancer Prevention with Hospital Staff:

A Pilot Project for System Change

\section{Introduction}

Childhood is an important time for positive behaviors to be in introduced. Health care providers working directly with children and adolescents are positioned to affect behavior change. Sun protection is especially important during early development because of potential overexposure to ultraviolet radiation while playing outdoors. Melanoma skin cancer is responsible for over 9,000 new skin cancer deaths every year, with incidence and mortality rates predicted to steadily increase in the United States though 2030 (Centers for Disease Control and Prevention, 2015). According to the Skin Cancer Foundation (2017), more people have had a skin cancer diagnosis over the previous three decades than all other cancers combined, and approximately one in five Americans will potentially develop melanoma skin cancer during the course of his or her lifetime. The cost of treating skin cancer is approximated annually in the United States at $\$ 8.1$ billion; whereas, $\$ 3.3$ billion of that estimation is attributed to melanoma (Rogers, Weinstock, Feldman, \& Coldiron, 2015). Data related to pediatric diagnosis of melanoma are especially concerning. According to the American Cancer Society (2017), melanoma is responsible for up to three percent of all pediatric cancers. Ferrari and colleagues (2005), reported that childhood melanoma is often misdiagnosed as pigmented lesions; therefore, there is a delay in treatment up to $40 \%$ of the time.

\section{Background and Significance}

The most common causes of melanoma have been overexposure to ultraviolet (UV) radiation, and the United States Department of Health and Human Services (2014) declared ultraviolet radiation as a human carcinogen. Although much of the exposure had come from natural sunlight, there had been a significant increase of melanoma reported because of indoor 
tanning exposure. In fact, studies had shown that over 419,000 cases of skin cancer annually in the US were linked to indoor tanning with 6,200 reported as melanomas (Wehner et al., 2014). The most critical point of understanding the information was this: sun damage is cumulative. Twenty three percent of lifetime exposure occurs by 18-years of age (Godar, Urbach, Gasparro, \& Van der Leun, 2003). Epidemiological research has shown melanoma as the most common skin cancer and $19^{\text {th }}$ world-wide (Ali, Yousaf, \& Larkin, 2013). There have been considerable amounts of variation with disease incidences between countries. For example, Australia has reported 37 cases per 100,000 population whereas South Central Asia reported 0.2 cases per 100,000 population, and many of the cases have been attributed to variations in racial skin phenotype and differences in sun exposure (Ali, Yousaf, \& Larkin, 2013). The majority of these cases $(98.2 \%)$ have been diagnosed among white-skinned individuals aged $\geq 65$-years with a median age of diagnosis at 57-years. Therefore, it has been imperative that youth be educated early and have the proper sun prevention health behaviors role-modeled and put in place to prevent the development of skin cancer, specifically melanoma.

Health care providers play a central role in conveying the importance of skin cancer prevention and sun protection awareness. A large-scale skin cancer screening initiative by Ferris et al. (2017) revealed skin cancer screening as a feasible initiative resulting in higher rates of melanoma detection among screened versus unscreened patients. Particularly, 333,735 patients were seen and only 53,195 received full-body skin evaluation. Of these 53,195 patients there were 50 melanomas diagnosed compared to 104 diagnosed from the unscreened 280,539 patients (Ferris, et al., 2013). This finding supported the need for health care providers to understand the importance of skin cancer screenings, and the need for further education supporting screening for all individuals. Robinson et al. (2004) suggested that health care providers' counseling could guide patient prevention practices, detection, self-efficacy, and knowledge related to skin cancer. 
In another study, Anderson et al. (2018) compared the diagnostic accuracy for skin cancer using physician assistants (PAs) and board-certified dermatologists. Those patients screened by a physician's assistant were significantly less likely to be diagnosed with a melanoma on site when compared to a dermatologist $(0.2 \%$ vs $0.4 \%$ of visits, $\mathrm{p}=0.04)$ (Anderson et al., 2018).

Conclusions drawn from these studies illustrated 2 points: (1) physicians and other health care providers lack specific education and training on skin cancer screening; and, (2) proper education on skin cancer prevention including counseling could help shape patient's prevention practices.

Understanding the need for sun protection counseling guidelines, the United States Preventive Services Task Force (USPSTF) in 2018 advised behavioral counseling concerning the reduction of exposure to ultraviolet radiation to reduce the risk for skin cancer in children, adolescents, and young adults aged 10 to 24 years. With adults $\geq 24$ years having fair skin, the USPSTF selectively recommended providing this service (USPSTF, 2018). The USPSTF also recommended that a health care provider speak with young individuals concerning the dangers of UV rays and how to prevent skin cancer during office visits. Also, the provider may encourage the children to take simple actions to protect themselves from the damaging effects of UV rays such as: using a broad spectrum sunscreen (protecting against both UVA and UVB rays) with a sun protection factor (SPF) of 15 or greater, covering the skin with a wide-brimmed hat, sunglasses, long-sleeve shirt, and a long pair of pants or longer skirt, avoiding direct sunlight between the peak hours of 10:00am and 3:00 pm when the UV rays are strongest, and avoiding indoor tanning booths or sun lamps (USPSTF, 2018). Previous to the 2018 Skin Cancer Behavioral Counseling guideline, the USPSTF examined the benefits of skin cancer screening with the adult population. The conclusion from the 2016 USPSTF Skin Cancer Screening guideline had shown insufficient evidence available to assess the balance of benefits and harms 
of currently providing this service, and therefore more research would be needed to support this recommendation.

In 2014, the US Department of Health and Services (USDHHS) issued the Surgeon General's Call to Action to Prevent Skin Cancer. This report solidified skin cancer as a major public health problem, and why we as a society, must act fervently to decrease incidence, mortality, and the economic burden related to the problem within our population. The report further addressed ways in which we can reduce the risk of skin cancer through communities and schools, outdoor work settings, and with both state and federal policies, legislation, and regulations. In essence, the Call to Action outlined five goals:

1. Increased opportunities for sun protection in outdoor settings;

2. Provided individuals with the information they need to make informed healthy choices about UV exposure;

3. Promoted policies that advance the national goal of preventing skin cancer;

4. Reduced harms from indoor tanning; and,

5. Strengthened research, surveillance, monitoring, and evaluation related to skin cancer prevention.

Many of the goals of the Surgeon General's Call to Action to Prevent Skin Cancer had already been addressed in the state of West Virginia. For example, on April 9, 2017 the WV Senate (SB 672) joined the House (HB 2520) in passing a law to restrict indoor tanning for anyone under the age of 18-years (West Virginia Legislature, 2017). According to the WV Cancer Burden Report (2016) issued by the WVU Cancer Institute for the USDHHS, melanoma skin cancer had remained in the top 10 cancers between 2009 and 2013.

The American Society for the Dermatologic Surgery Association (ASDSA) issued a white paper position statement on sunscreen use in schools (2013). The position statement 
encouraged statewide policies that would allow students to apply, carry, and store sunscreen for personal use in school, as well as supported educational initiatives designed to encourage and promote safe, smart sunscreen use among all students. According to the ASDSA (2013), major barriers to sunscreen use in schools were the lack of physician support allowing students to apply, carry, and store sunscreen for personal use, and potential policies inhibiting sunscreen from being given to students by school district officials (otherwise opposed by a parent or guardian). According to the Skin Cancer Foundation (2017), one blistering sunburn during childhood or adolescence more than doubles the chance of being diagnosed with Melanoma later in life. Unfortunately, West Virginia had not been included among the states previously considering sunscreen availability in the school system.

\section{Problem Statement and Project Purpose}

Because sun protection during outdoor exposure is imperative in health promotion and skin cancer preventive measures, health care professionals and staff need to be provided with evidence-based educational interventions to enable them, in turn, to educate their own patients about sun protection. Little literature exists examining sun prevention education interventions and hospital staff. The purpose of this project was to implement an educational intervention using a pre-determined curriculum from The Skin Cancer Foundation to increase hospital staff knowledge regarding sun preventive measures. This project has been implemented using the following methods:

1. Collaboration with the directors of education within the facility to coordinate curriculum intervention;

2. Education of staff about the application of sunscreen and other sun preventive measures; 
3. Communication of goals regarding sun protection and health promotion to all stakeholders; and,

4. Provision of staff support to ensure intervention sustainability post implementation.

The results of the project have offered evidence to the body of advanced practice nursing knowledge related to skin cancer prevention. After implemented and successfully sustained within the organization, the results will contribute to the knowledge base related to early intervention and education regarding sunscreen use by patients in direct care facilities. The results of the project also have potential to assist health care providers to improve health promotion outcomes throughout the lifespan; thus, decreasing morbidity and mortality in melanoma skin cancer and decreasing health care costs.

\section{Literature Review and Synthesis}

Four literature reviews were conducted for this proposal: (1) sunscreen interventions and programs implemented in school-based or community settings; (2) institutionalized children and health outcomes; (3) staff educational interventions and sun protective behavior; and, (4) intent to change behavior and educational intervention. Because of the unique nature of the proposed project and lack of evidence-based interventions, the combination of the four searches produced satisfactory results warranting further examination to support a pilot study for this particular focus. Thirteen databases were rigorously searched to cover the breadth and depth of the topic area. These included: Academic Search Complete, CINAHL, Education Research Complete, ERIC, Health and Psychosocial Instruments, Health Source (Consumer Edition and Nursing Academic Edition), MEDLINE, PsychARTICLES, PsychINFO, Social Work Abstracts, TOPIC search, and Cochrane Library. Search terms and Boolean phrases for all databases were “institutionalized children and sunscreen", “institutionalized children and health intervention", "looked after children and sunscreen", "looked after children and health outcomes", "health care 
professionals and sun preventive education", "hospital staff and sunscreen", "health care professionals and sunscreen", and "health care professionals and sun protection interventions", and "intent to change behavior and educational intervention". Limitations included English language, peer reviewed publications in or after 1990 to the current period of the review, no age restrictions due to staff and child focus for educational intervention; and, abstract inclusion only.

The Cochrane Library produced one hit, and the total number produced from all other databases included 237 results. Each of the 237 articles were reviewed, and 18 were deemed appropriate for inclusion criteria to support the current clinical change project including 2 systematic reviews, 9 randomized controlled trials, 3 quasi-experimental studies without randomization, 2 descriptive cross-sectional, 1 repeated-measures, and 1 descriptive correlational study.

The systematic review conducted by Maliessye et al. (2013) looked at 15 studies (1 RCT, 2 cohort, and 12 cross-sectional) which examined the association between melanocytic nevi, or moles, in childhood and sunscreen usage. Findings had shown 12 studies lacked reporting of sunscreen's protective effect against the development of melanocytic nevi; however, 3 studies reported a melanocytic nevus count which lowered with sunscreen application. According to the authors, the differences in the various studies' results could have been attributed to variations in melanocytic nevi counts, sun overexposure, and insufficient sunscreen application on fairskinned children. Further research would be needed with epidemiological studies where the data would be homogenous in nature and would therefore make meta-analyses possible. In conclusion, overall findings had shown no evidence of the protective effect of sunscreen on melanocytic nevi development in children; however, the authors may not have considered the number of melanocytic nevi that can develop later in adulthood from sun overexposure during childhood. Because childhood overexposure to sun and inappropriate sunscreen application had 
contributed to skin cancer development in adulthood, this systematic review had been included to support the proposed capstone.

Lee, Rivers, and Gallagher (2005) conducted a randomized controlled trial evaluating the impact of sunscreen application on noncancerous nevus development among white school children. The authors concluded that there were significant differences between school-aged children from 6 to 10 years of age in the development of new nevus on the trunk when given sunscreen of SPF 30 and parental instructions and/or self-directed usage in the intervention versus the control group. Nevus were counted ranging from $2 \mathrm{~mm}-5 \mathrm{~mm}$ or greater and were counted by the same group of specially trained physicians (blinded) to assist with minimizing biased results. The study was followed for 3 years after implementation, and results supported the use of sunscreens decreased the development of nevi in children, thus potentially reducing the risk for cutaneous malignant melanoma in later adult life.

Two randomized controlled trials examined the results of tailored interventions focused on the health prevention behaviors of children. Glanz, Steffen, Shoenfeld, and Tappe (2013) conducted the Project SCAPE Family Study (Skin Cancer Awareness, Prevention, and Education) which targeted children and their parents, and evaluated tailored sun protection interventions versus non-tailored. The family trial included an intervention group receiving multiple mailings of skin cancer prevention materials of a tailored nature, whereas the control group was provided with one mailing of standardized skin cancer informational material. The study included children in grades 1-3 and was conducted in two geographical regions of the United States from Hawaii to New York to include various ethnic groups and climates. Conclusively, the results supported a focus on tailored communication with both parents and children, with statistically significant improvements in use of sunscreen and other sun protective habits (e.g. wearing a hat, shirt, staying in the shade, and extended sun exposure). Skin self- 
examinations improved for both parents and children in the intervention group, indicating that behaviors derived early in the childhood years could potentially be adopted and practiced throughout adulthood. Ultimately, this study supports the current capstone proposal in that sun protective research in child care centers and schools could increase knowledge and use of sun protective measures.

Similarly, the CATCH-ON study was designed to examine the conditions under which a program is institutionalized after a trial has ended. Osganian, Parcel, and Stone (2003) performed the largest field trial, known as the Child and Adolescent Trial for Cardiovascular Health, of school-based health promotion in the United States, consisting of 96 schools in 4 geographical areas covering California, Louisiana Minnesota, and Texas. Although this trial was designed to examine the promotion of healthy lifestyles related to cardiovascular health, there was important information gleaned from this research lending value to the current capstone proposal. The authors stated that evidence-based public health promotion interventions including the school system should be considered a major venue in reaching youth and families. The authors continued to explicate that institutionalization of health promotion programs should aim intensive interventions at all levels of the organization including staff, administration, teachers, and support staff. This is essential for sustainability and program success. In essence, results from the $\mathrm{CATCH}-\mathrm{ON}$ study illustrate health promotion programs can be sustained in a school-based program with staff training, a program champion, and administrative support and resources.

In a fourth randomized controlled study, Gritz et al. (2007) stressed interventions increasing the sun protection of younger children should focus on their care providers partly because of their dependence on them for sun protective measures. Much like the population of focus at the organization in the current capstone project, the children are looked after by 
caregivers and are mostly dependent on them for health promotion and/or behavioral interventions. In the Sun Protection is Fun! (SPF) study, preschool staff knowledge was evaluated about behavioral and psychosocial outcomes associated with child protection from sun exposure (Gritz, et al., 2007). The intervention itself included training, a video, newsletters, as well as a curriculum including sunscreen. After baseline survey completion, the 2-year randomized groups were assessed again at 12 and 24 months. Findings indicated the staff in the intervention group were more likely to apply sunscreen to their students, carry sunscreen on field trips, and perform reapplication at the appropriate times of sun exposure. The strength of using caregiver education in looked after children supported future sun preventive interventions.

Also, in 2007, Naldi et al. conducted a cluster randomized trial examining an educational intervention to reduce sunburn rates and improve sun protection measures in Italian elementary school second and third grade children. Much like the study by Gritz et al. (2007), this research focused on an educational curriculum within a school system conducted by video-trained teachers who supplied the parents and children with booklets of sun prevention education. Eight thousand, six hundred and eleven children completed the 16-month study, and no significant difference was found in documented sunburn episodes between the intervention and control groups. Also, at follow-up, comparable sun protection practices were reported with both intervention and control groups. No significant differences emerged for melanocytic nevus count between the subgroups. Unlike the Gritz et al. (2007) study, there was no significant influence of the proposed educational program 1-year post follow-up. The authors proposed that the inability to achieve the desired outcome of decreasing sunburn rates and improving sun protection measures could be due to the high level of protection currently present in the population. Seventy one percent reported sunscreen use regularly; therefore, the authors argued against proposing generic educational interventions in schools involving written material 
distribution and short-curriculum application to improve sun protection behavior. The population under study in the current capstone project currently has no skin protection; therefore, the results of the study may reveal different outcomes after the Sun Smart U education is put into place.

Dietrich et al. (1998) and Dietrich et al. (2000) conducted 2 randomized controlled trials focusing on community-based interventions that encouraged sun protection for children. The first study included 10 towns in New Hampshire that were paired and then randomly assigned to intervention or control groups. This SunSafe multicomponent intervention was given to both caregivers and children in the following settings: primary care practices, day care centers, schools, and recreational beach areas. School interventional groups were evaluated based on 1 of 3 components: (1) a visit to the principal providing in-services to staff and a parent outreach program, (2) one project staff visit, or (3) curricular visits via mail with no project staff visit. The primary practice component involved meetings for both clinician and staff focusing on sun protective education, posters, counseling, and SunSafe tools (e.g. stickers, removable tattoos, etc.) for dissemination to children during well-child visits. Finally, the community recreational intervention involved in-servicing lifeguards at beaches by providing them with daily UV index reports for display, and free sunblock samples made available to beachgoers via lifeguards. The 1,930 children participants were observed using sun protective practices such as sunscreen use, shaded areas for play, and protective clothing. Results had shown that use of sunscreen on at least one body area increased substantially in all 5 interventional towns when compared to control groups. Conclusions from this study support sun protection behavior in children could be enhanced with the SunSafe interventions provided to schools, primary care centers, and recreational beach areas. 
A follow-up study conducted by Dietrich et al. (2000) on the previous randomized controlled trial was performed to evaluate children's sun protective behaviors 1 year post initial intervention. In the town intervention groups, results had shown sustainability of the initial intervention with an increase in the proportion of children using some sun protection. The authors stated that this increase was in direct proportion to sunscreen usage. Also, of significance in this study, caregivers of children in the intervention towns admitted receiving additional sun protection information from the health care sources and schools $(62 \%)$ versus the caregivers in the control town $(33 \%)(\mathrm{p}<0.006)$. In conclusion, this follow-up study confirmed a persistent increase in children's sun protection through community intervention efforts, recreational beach areas, and school involvement which supported the need for SunSafe education in institutionalized children in a health care setting.

In a quasi-experimental study, Hoffman, Rodrique, and Johnson (2000) used the theory of reasoned action as a conceptual framework in examining the effectiveness of a school-based program to enhance knowledge of sun exposure and attitudes of sunscreen use among children. The study population included 181 fifth-grade students selected from 8 science classes in 2 different elementary schools in north central Florida ( $n=99$ intervention group, $n=82$ control group). The intervention group received (1) factual information regarding the sun's effect on skin in a lecture format followed by visual materials focusing on the negative effects of sun exposure, (2) a homework assignment for each child to create a sunsafe poster for the classroom, and (3) a show-and-tell session of the posters with fellow classmates. Results show a significantly higher change in knowledge scores, greater trend toward positive attitudes and beliefs on sun protective practices, and greater change in intention to practice sun protective behaviors when compared to the control group. This study supports that a 3-4-day intervention 
in a school-based setting was effective in increasing sun protective behaviors and intentions among school-aged children.

A study conducted by Stanton, O'Riordan, and Roy (2003) examined the variation in levels of sun exposure and environmental conditions in children aged 3 to 5 years. In this repeated measures design, a sample size of 49 children from Brisbane, Australia were exposed to ultraviolet radiation (UVR) under 4 conditions: sunny days, cloudy days, teacher's instruction to stay in the shade on a sunny day, and sunscreen application instructions provided by a healthcare professional on a sunny day. The sample population was chosen because the authors' indicated that a lack of research existed on children of this age who were not attending school. Therefore, dependency on the caregiver and environmental conditions under which the children were exposed should play a significant part in the effects of sun exposure. Data collection methods included completion of a questionnaire or a diary by the parents and researchers, observational audits via video recording, and polysulphone dosimeter readings from badges attached to the wrists of the children upon entering the care centers. Reports from the parents indicated over $80 \%$ of the children had a history of sunburn. Outside playtime was recorded between the hours of 9:00 and 10:00 a.m. due to the decreased intensity of the sun. Dosimeter readings were reported higher on sunny days than in the other 3 condition groups $(\mathrm{p}<0.05)$, and there was a significant effect $(\mathrm{F}=3.6, \mathrm{p}=0.004)$ that absolute sun exposure decreased during the days where the children were instructed to stay in the shade. Sunscreen was applied to all children to the center prior to the outdoor exposure period. Conclusions of this study support efforts to educate child caregivers in factors contributing to sun exposure, and sun-safe practices which need to be maximized for children of this age group because skin cancer prevention and early detection can be key in avoiding the disease for the future. 
Darling \& Ibbotson (2002) performed a descriptive cross-sectional study that examined the attitudes, knowledge, and behavior of health care professionals and the general public toward sun awareness issues. With a convenience sample size of 108 participants including health care professionals and the general public (doctors $=28$, nurses $=28$, general public $=52$ ), results had shown that doctors have a significantly higher level of knowledge regarding sun exposure hazards, sunscreen labeling, and UV radiation terminology $(\mathrm{p}=0.2)$. Dermatology staff were purposefully excluded from the study due to their specialty knowledge of skin cancer and sun protection behaviors. Instrumentation used was a closed-nature questionnaire format with a 74\% response rate from health care professionals, and a 100\% response rate from the conveniently sampled public participants. There were no significant differences found between the groups for 'seeking a tan' and 'recalling a tan'. Of the 28 doctors $14 \%$ recalled a sunburn experience. This was reported as significantly less $(\mathrm{p}=0.03)$ than non-tan seeking nurses $(50 \%)$ and the general public (47\%). Although this study lacked randomization, the findings support the education of health care professionals regarding sun specific interventions to promote behavior change for themselves and their patients.

Another study conducted by de Troya-Martin et al. (2016) focused on identifying main risk factors for sunburn and planning future interventions aimed at healthcare professionals for the promotion of skin cancer prevention. This cross-sectional descriptive study was conducted in Costa del Sol, Spain, and focused on the sun exposure, protection practices and habits, and attitudes to tanning and sun protection (healthcare professionals $=450$, nurses $=226$, doctors $=224$ ) in a primary care setting. A previously validated self-administered questionnaire (Beach Questionnaire) revealed $15.3 \%$ of doctors compared to $30.9 \%$ of nurses participated in beach exposure 30 days per year $(\mathrm{p}<0.001) ; 52.4 \%$ of hospital staff showed significant differences in sunburn events when compared to $30.4 \%$ of primary care staff. Similar differences were also 
found with attitudes toward use of sunscreen $(\mathrm{p}=0.014)$. This study provided support to the current project due to the content focus areas of primary care versus specialized care regarding sun behaviors. The outcome shows that specialist healthcare providers are more likely to suffer from sunburns and needed encouragement to change sun exposure attitudes and habits.

Another component of this project was to examine one's intent to change health care behaviors, and how that could affect actual behavior change. Godin, Belanger-Gravel, Eccles, and Grimshaw (2008) published a systematic review of scientific literature examining factors influencing healthcare professionals' behaviors and intentions to adopt a clinical-related behavior grounded on social cognitive theories. An examination of 5 prominent data bases and theses indexes were searched between September 14 and October 30, 2007. For analysis purposes the weighted mean $\mathrm{R}^{2}$ was calculated for behaviors and intentions, and variables contributing significantly $(p<0.05)$ to the prediction of intention and behaviors were documented. Of the 78 studies meeting inclusion criteria, 72 spoke to the determinants of intention, and 16 spoke to the determinants of behavior. Two theories were consistent through the included literature: (1) Theory of Reasoned Action (TRA); and, (2) Theory of Planned Behavior (TPB). Results indicated a weighted mean $\mathrm{R}^{2}$ of 0.31 for observed behavior prediction, and $\mathrm{R}^{2}$ of 0.59 for prediction of behavioral intention. This systematic review implies the TPB is more appropriate in predicting behavior; although, other theories such as Theory of Reasoned Action, Triandis' theory of interpersonal behavior, and Bandura's social cognitive theory would better explain intention. From this work, it can be surmised that healthcare professionals consider role beliefs and moral norms as important variables determining his or her intention to adopt a clinical behavior.

Pertl et al. (2010) investigated individuals' perceived control and self-efficacy on his or her intention to engage in skin cancer health behaviors. This quasi-experimental study focused 
on previous research based on the Theory of Planned Behavior (TPB) and the lack of empirical evidence connected the perceived behavioral component (PBC). The study had 2 aims: (1) to examine skin cancer behaviors in the dimensional construct of the perceived behavioral component; and, (2) to predict sunscreen and sunbed intentions in relation to self-efficacy. Young adults between the ages of 16 and 26 years were recruited from the general public around various locations in Ireland between December 2007 and January 2008. This convenience sample yielded 590 participants (male: 236, female: 354) who were given questionnaires asking frequency of both sunscreen and tanning bed usage. These Likert scale items measured both intent and attitudes towards sunscreen and/or tanning beds usage, as well as the subjective norms for each behavior.

Results in this study sample indicated that low sunscreen usage was reported by one-fifth of the participants, and approximately half used it occasionally on sunny days (Pertl et al., 2010). The most popular occasions mentioned for sunbed usage were weddings (40\%) and vacationing (55\%); however, $45 \%$ of the total sample reported being sunburned 'easily' or 'very easily' (Pertl et al., 2010). The Theory of Planned Behavior correlated significantly with both intentions to use sunscreen and sunbeds (Ps $<0.01)$; hence, those with fairer skin reported higher intentions $(\mathrm{r}=-0.14, \mathrm{P}<0.01)$ and positive attitudes $(\mathrm{r}=-0.22, \mathrm{P}<0.01)$ toward sunscreen, but the opposite was true for intent toward sunbed usage $(r=0.10, p<0.05)$. Conclusions of the study revealed that self-efficacy was a significant predictor of sunscreen usage; however, controllability significantly predicted intention of sunbed usage. Findings from this study support that the decision to participate in both sunbed usage and sunscreen is a personal decision and is well within the individual's control - should the individual perceive the self-ability to do so. 
McComb, Jones, Smith, Collins, and Pope (2016) conducted a study probing college students' intent toward engaging in healthy diet behaviors using a cross-sectional randomized controlled design. The research compared the effectiveness of 4 virtual interventions on healthy eating behaviors, and the ability to shift the college students' intention to engage in those behaviors. Also referencing the Theory of Planned Behavior (TBP), McComb, Jones, Smith, Collins, and Pope (2016) reiterate the 3 functions that predict behavior are attitudes, perceived behavioral control, and subjective norms. The authors also mention literature on the constructs of the TBP perform better than other health models (e.g. Health Belief Model) in predicting both behavior change and intent. A convenience sample of students $(\mathrm{N}=232)$ used in the Midwest were randomly assigned to 4 virtual experimental conditions using a pre-designed questionnaire given both before and after the intervention. Questionnaires measured demographic characteristics, dietary habits and knowledge, body mass index (BMI), and the above mentioned TBP constructs. Behavioral intent was measured on a 7-point Likert scale (e.g. agree-disagree) using 3-item questions focusing on the participants' intent to engage in health eating behaviors. While the intervention showed a significant increase $(p<0.05)$ in perception of eating healthier diets using coupons and/or coaching, attitudes of eating fruits and vegetables were not significantly affected. In measuring intent, attitude was the only TPB construct significantly influenced ( $p=0.009$ ) when compared with students' wellness focus, delivery method, or both in changing dietary behaviors (McComb, Jones, Smith, Collins, \& Pope, 2016). This study supports that the intent to engage in healthy dietary behaviors is influenced by all TBP constructs, with the exception of attitudes focusing on fruits and vegetables.

Morwitz, Johnson, and Schmittlein (1993) performed original research on the mere measurement hypothesis, which is noteworthy of discussion for the purposes of this project. This indicates that by merely measuring one's intent to engage in a behavior, will in fact, 
increase the act of that behavior. In a longitudinal quasi-experimental study examining the impact of intent on purchase behavior, Morwitz, Johnson, and Schmittlein (1993) proposed that (1) the intent of asking a question does impact purchasing behavior, (2) purchasing behaviors differs in regard to number of times asked; and, (3) purchase behaviors are less affected if there are no prior familiarities with the product being purchased. Using retrospective data, 7 separate mailed waves of surveys between July 1986 and January 1989 asked household consumers about intent to purchase an automobile or personal computer (PC) in the future. Results show that households asked about automobile purchase intent actually purchased $37 \%$ more (in the next 6 months) than those households not asked about purchase intent $(\mathrm{p}=0.0069)$. In addition, asking persons about PC purchase intent one time increased the purchase rate from these households by $18 \%(\mathrm{p}=0.0735)$. In summary, more products were proportionately acquired by households that were asked about intent to purchase compared to those households not asked about intent to purchase. This study lends evidence to the possibility that those individuals asked about intent to engage in sun preventive behaviors could be cognitively inclined to do so just by mere suggestion.

Complimenting the theory of Morwitz, Johnson, and Schmittlein (1993), Levav and Fitzsimons (2006) performed 3 experimental randomized controlled trials also examining the mere-measurement effect on intent to change personal behaviors. Different from the previous study, Levav and Fitzsimons (2006) added the investigation of cognitive work a person must engage in while responding to questionnaires regarding intent. The first experiment randomized MBA students $(\mathrm{N}=145)$ into one of three conditions: (1) probability of pleasure reading within the next 2 weeks, (2) probability of flossing his or her teeth in the next 2 weeks; and, (3) probability that a classmate would floss his or her teeth in 2 weeks. Those in the self-intent group flossed a significantly greater amount when compared to the control group $(6.25$ vs $4.11, t(96)=$ 
$2.06, \mathrm{p}_{\text {rep }}=0.89, d=0.42$ ). Therefore, the easier it was for the participant to imagine themselves performing the behavior, the more intention there was to engage and perform the behavior. In the second experiment (N=99), Levav and Fitzsimons (2006) manipulated ease of representation by varying the time frame; hence, the participants were either (1) asked a positively framed question regarding intent directly, (2) a question about the probability of no intention in directly engaging in behavior, (3) or the probability of avoiding it altogether. The different groups randomly assigned to groups and asked (Likert scale) about intent to consume fatty foods, not consuming fatty foods, avoiding fatty foods, or consumption of orange drinks. Results indicated $92 \%$ in the control group (consumption of orange drinks) chose the fatty foods over the healthy food. Experiment 3 tested ease-of-representation whereby a mere-measurement effect for regularly performed behaviors would be more evident than for irregularly performed behaviors. Random assignment here $(\mathrm{N}=63)$ included randomization to (1) probability of flossing in the upcoming week, or (2) reading for pleasure in the upcoming week. The target of regularity was indicated by either behavior being performed 7 to 21 times vs 2 to 8 times in the upcoming weeks. Results indicated significant interaction reported for flossing, $F(1,59)=7.92$, prep $=0.96, d=0.73$ (Levav \& Fitzsimons, 2006). This study supports that regularity and frequency of questioning can affect self-reported behavior that only happens at regular intervals. Conclusions from this study indicate that the more mentally difficult it is to imagine oneself engaging in the behavior, the less regularly it will occur. Thus, mere-measurement effect suggests that intent to engage in behavior is easier to perform when there is a positive self-mental representation of performing the act.

A quasi-experimental study by Davis et al. (2015) examined the effect of a 'Students Are Sun Safe' (SASS) program delivered by college students to middle-school and high-school students. The authors suggest because adolescents are an important population to target for sun 
prevention and protection behaviors, project SASS would focus on the adolescents' perceptions, behaviors, knowledge, and intent to change behaviors post intervention. The teachers were asked to complete reviews regarding program delivery, whereas the students completed the pre and posttests along with a satisfaction questionnaire. The Health Belief Model (HBM) was used as a framework to measure the adolescents' perceptions on susceptibility to skin cancer, severity of UVR damage, benefits of UVR avoidance, and berries to sun safety (Davis et al., 2015). Originally developed at the University of Arizona, this evidence-based project is delivered in the classroom and lasts approximately 50-65 minutes. Project SASS was delivered to nine schools $(\mathrm{N}=1,284)$ in 5 districts of Tucson, Arizona ( 5 middle schools, 4 high schools). After the intervention, students perceived themselves at a higher risk for skin cancer during their lifetime ( $p=0.01)$ (Davis et al., 2015). Post intervention, middle-schoolers reported less time in the sun; however, tanning behaviors reported by high-schoolers post intervention showed no change $(p=0.84)$. Overall, there was a $74.4 \%$ increase in knowledge for skin cancer recognition, $76.9 \%$ increase in identified UVR factors, and 67.6\% increase for appropriate detection of sun protection behaviors (Davis et al, 2015). In summary, findings reported positively in adolescent perceptions of tanning, knowledge, and intentions for self-behavior change. The authors note that limitations may have included self-reporting by the participants, as well as peer influence or social pressures impacting this target population.

\section{Synthesis and Conclusion}

When analyzing the literature, there were findings with both consistency and variation, as well as identified gaps where further research is needed. Similarities were discovered that supported both the education of health care professionals with sun protective practices, and early childhood implementation of sun preventive measures. When considering such preventive programs, child care centers or other educational institutions should consider essential 
components for sustainability such as including parents/caregivers as well as tailored interventions for health care professionals to yield positive outcomes. Differences in the literature were apparent through the assorted definitions and terms including 'sun exposure' and 'inappropriate us of sunscreen'. Variations in conceptual definitions can blur result comparisons when identifying the significance of study outcomes for specific practice change. Also, the discretionary use of sunscreen by geographical location would vary because some regions may have increased awareness of sun protective behaviors due to advertising, distance from the equator, and/or social acceptability of engaging in such behaviors. Parent and/or caregiver lack of supervision regarding sun protective measures was also identified as a variation factor.

The intent to change behavior has been examined extensively using the Theory of Reasoned Action (TRA), Theory of Planned Behavior (TPB), Triandis' Theory of Interpersonal Behavior, and Bandura's social cognitive theories. Literature supports Bandura's social cognitive theory as a better explanation for intention to change behaviors (Godin, BelangerGravel, Eccles, \& Grimshaw, 2008). In addition, a person's self-efficacy and self-reliability are also predictors of intention to engage in sun protection behaviors. For the purposes of this project, discussing the mere measurement effect (Morwitz, Johnson, \& Schmittlein, 1993) is noteworthy of mention as it lends evidence to the possibility that individuals asked to engage in certain behaviors are more likely to do so because of cognitive suggestion; and, intent to engage in the behavior is easier to perform when there is a positive self-mental representation present (Levav \& Fitzsimons, 2006).

The gaps identified from the review show lack of a standardized protocol for attaining sun awareness levels in health care professionals, or even the general public. Because the methods of evaluating sunscreen's effectiveness as a means of childhood malignant melanoma development were varied, researchers suggested further epidemiological studies be conducted 
with a standardized protocol of measurement (Maleissye, et al., 2013). The essential components of sustainability involving staff and caregivers in long-term education need to be addressed, as well as how the staff behavior impacts child behavior practices regarding sun preventive measures. This review suggests sunscreen usage has long-term impacts for children and health outcomes, and children can perhaps be influenced by the healthcare providers' knowledge regarding sun protective practices and skin cancer awareness.

\section{Theoretical Framework}

The ultimate goal for this project was to implement an educational intervention to measure hospital staff knowledge of sun preventive measures. The aim of this intervention was to assist health care professionals in the decision-making processes while directly caring for patients during outdoor recreational time in a residential facility when UV rays provided peak exposure. The nurses and other staff in charge of patients were educated about sun safety, sunscreen applications, and skin monitoring during outdoor exposure. Because this was a new educational intervention for the facility, there was a process of change that the personnel needed to put into place to ensure proper implementation among health care personnel. It was imperative that organizational change happening on a large scale be correctly assessed, implemented, and evaluated to anchor new behaviors rooted in the culture, shared norms, and shared values of affected people.

John P. Kotter's theory of leading change was the model utilized in the design of this project. Kotter developed this theory in the late 1990's and based it on an analysis of multiple initiatives over the previous fifteen years that attempted to produce significant organizational change by way of restructuring, reengineering, re-strategizing, downsizing, acquisitions, quality programs, and cultural renewal (Kotter, 1996). He continued to develop his theory after watching transformation efforts fail within major companies. Kotter (1996) developed 8-steps to 
leading and sustaining successful organizational change. In developing this theory, Kotter (1996) pointed out the obvious reasons that transformational change fails. These included allowing too much complacency within the organization, failing to create a powerful guiding coalition, underestimating the power of vision, under communicating the vision by a factor of 10 , permitting obstacles to block the new vision, failing to create short-term wins, declaring victory too soon, and neglecting to anchor changes firmly within the corporate culture (Kotter 1996). The first step in Kotter's model (1996) is establishing a sense of urgency. During this initial stage, it was imperative the change agent institute a sense of urgency that overpowered high levels of complacency. If complacency levels were elevated, transformational efforts would more than likely fail because of the lack of people interested in working on changing the problem or issue. Kotter (1996) suggested one needed to identify the sources of complacency (if present), to create a sense of urgency for the needed change. Examples of sources of complacency included the absence of a major and visible crisis, too much happy talk from senior management, low overall performance standards, and a lack of sufficient performance feedback from external sources. Therefore, in order to push up the urgency level, the sources of complacency must be removed to minimize their impact on the proposed change. There was no specific sun preventive education provided to the hospital staff at the facility where this project had been implemented, nor documented measures taken with the patient population during outdoor recreational activities. Because health care professionals have an ethical responsibility to provide patients with the tools to achieve optimal health promotion, there was a justified cause for a staff educational intervention that addressed sun prevention within this facility.

There must be a group put into place with enough power to effectively lead the change. Therefore, Kotter's (1996) second step referred to creating the guiding coalition. This step involved both identifying who needed to be a part of the group, as well as getting the group to 
work together like a team. Although a strong leader was recommended, Kotter (1996) did not recommend tackling change in isolation; a strong coalition with the right trust composition and shared objectivity was an essential part of the early stages of change. The essential characteristics of team members should include key players in positions of power, individuals with expertise in the discipline of the change topic, people with credible reputations for getting the job done, and proven leaders to drive the change process. Considering the organizational structure of the facility and the proposed project, the guiding coalition consisted of nursing and behavioral health leaders from the residential units, the Medical Director, education personnel, and of course the CEO who helped inspire and push the vision of the project. These members were also essential in the day to day operations of the organization and conveyed both strong managerial and leadership skills on the guiding coalition.

The third and fourth steps in leading change are developing and communicating a vision and strategy to help direct the change effort. Kotter \& Cohen (2002) pointed out clarifying the direction of change was important to keep confusion at a minimum, thereby limiting questions of whether the change was necessary. A vision should be so clear that it can be articulated in less than one minute, or written on one page (Kotter \& Cohen, 2002). Vision assists in aligning individuals, therefore coordinating efforts of motivated persons in an efficient way. According to Kotter (1996), it was this specific characteristic of vision that was vitally important. Without coordination of efforts, people would not own a shared sense of direction. This way, a shared vision allowed the group to work interdependently with some degree of autonomy, therefore introducing empowerment and ownership of the change project. To convey the vision of implementing an evidence-based educational intervention for hospital staff, the project plan focused on the importance of sun prevention in the current patient population. Also, this vision was communicated to staff and change agents throughout the facility using Sun Smart U (The 
Skin Cancer Foundation, 2017) literature, educational sessions, and "every vehicle possible", (Kotter, 1996, p. 21). Educational sessions were offered at various times to incorporate all shifts in the facility and encouraged question and answer sessions to clarify the overall goal of protecting patients from harmful UV rays while under the careful monitoring of the health care team. It was also important during this process that key leaders role modeled this change so that others would see its importance and significance to the organization.

Empowering broad-based action and generating short-term wins are steps five and six of Kotter's change theory. Empowering broad-based action involved getting rid of obstacles that may have prevented the change from taking place in the organization. In addition, this step also encouraged changing any systems or structures already in place that could undermine the vision of change. Kotter (1996) suggested encouraging risk taking during this phase, and proposing nontraditional ideas, activities, and actions. Enlisting hospital-wide champions, including physicians and staff, assisted in the development and implementation of a sunscreen policy initiative in this institution. To accomplish this, training sessions were given using the Sun Smart U tool kit provided by the Skin Cancer Foundation (2017). This information was tailored to middle and high school populations and corresponded with the demographics of the current facility. Because this tool was easy to understand and incorporated fun into learning and practicing sun safety, it should have been easily understood and adopted by the hospital staff which is the population of focus. In addition, generating short-term wins should have been visible evidence of the change taking place within the organization. For instance, a reward system may eventually be put into place that celebrates the unit displaying $100 \%$ participation in sun prevention activities during the month of May, etc. Creating and following through on the "wins" would have been imperative to ensure the change was real and taking place throughout the culture of the organization (Kotter, 1996). By visibly recognizing and rewarding individuals 
who made the wins possible, the success of the project's implementation will more likely achieve sustainability and provide a sense of ownership among staff and providers.

Promoting and developing people who can implement the change vision is one way to consolidate gains and produce more change, which is the seventh step in Kotter's theory. Major change in any organization takes time; however, under the potential circumstances of bad luck, turnover of key change agents, and exhaustion of the leaders, the celebration of short-term wins can decrease momentum and breed complacency (Kotter 1996). In order to consolidate gains and produce more change, the change process must be reinvigorated with new projects, themes, and/or change agents that can continually develop the vision forward (Kotter, 1996). During the phase of project implementation this step can be accomplished by highlighting new staff members that show initiative to be promoted to take part in the project. If short-term wins are celebrated, and those who have participated are openly rewarded for his or her accomplishments in projecting the change forward, then more participants may feel empowered to take part in something bigger than themselves. Thus, the process would have new ownership and fresh energy to continue.

Kotter's (1996) final step in leading change eludes to anchoring new approaches in the culture. After the end product of change has been successfully established, it must be firmly grounded in the organizational culture in order to continually be accepted as practice standard. There may be regression behaviors appearing as incremental; however, the postmortem probability here is that the organization's culture could be incompatible with the final change result. Kotter (1996) insisted that connections between new behaviors and organizational successes must be articulated to employees. In other words, those involved in the day-to-day practices of carrying out the product of the final change must be shown or convinced that the positive outcomes and results are because of these new behaviors or processes that have been put 
into place. Culture is very powerful in an organization because it gives the employees a sense of shared values and pride in the workplace. If new practices are incompatible with the culture, regression is highly plausible. The organization where the project was implemented holds the following values: quality care in a patient-centered environment, innovation, ethical treatment of all, fiscal responsibility, total well-being, investment in people, and visibility. The project of implementing an educational intervention for hospital staff regarding sun prevention with the patient population was consistent with both the culture and mission of this organization.

Because the purpose of the project was to protect the patients and staff members and provide them with quality care that is evidence-based in nature, this final step of the change process should be fully adopted and accepted by those within the organization.

\section{Project Description}

\section{Evidence-Based Design and Intervention Plan}

The proposed pilot project focused on using a predeveloped sun safety awareness tool to educate the health care professionals and staff working with institutionalized children on the risks of skin cancer related to sun overexposure. Four measurable objectives included (1) the staff's baseline level of knowledge regarding sun protective behaviors, (2) the staff's level of knowledge regarding sun protective behaviors post educational intervention with Sun Smart U Curriculum, (3) the staff's intent to change behavior; and, (4) the feasibility of implementing behavior change within the organization. The actual intervention with the staff was to include one 40-50-minute session delivered face-to-face using a multimedia approach. The staff was asked to provide standard demographic information for reporting purposes only, and also provided with a questionnaire regarding perception of implementation within daily practice.

As identified in the literature search for this project, a previous study performed by Dietrich et al. (1998) and Dietrich et al. (2000) conducted 2 randomized controlled trials 
focusing on community-based interventions that encourage protection for children. Children were provided with a SunSafe intervention through the school systems (in addition to day care centers, primary care offices, and beach recreation areas). Results had shown a use of some sunscreen on at least one body area which increased in all 5 towns included in the intervention, which was a proportional increase of 0.56 from those observed at baseline to 0.76 post intervention. The SunSmart programs utilized in the project were structured to meet regional needs and delivered by teachers in a classroom type setting. The authors stated this increase was in direct proportion to sunscreen usage and encouraged an intervention similar to this one be tested in other areas to show efficacy for wider dissemination in schools, health care centers, departments of health, and professional organizations. Although the aforementioned study focused on the observation of 1,930 children in 10 townships, and was coordinated over 15 schools, 14-day care centers, and 6 Head Start programs there was much to be gleaned from this study as a good base of evidence to support the staff education regarding sun protective behaviors and skin cancer awareness.

Because of the advancements in medicine, science, and technology, there have been enormous amounts of literature emerge regarding the importance of skin protection to avoid overexposure to UVA and UVB rays for the prevention of skin cancer. From the Surgeon General's Call to Action to Prevent Skin Cancer (USDHHS, 2014) to the declaration of the entire UV spectrum and UV-emitting tanning devices being classified as human carcinogens (Ghissassi, et al., 2009), there is a documented need for educational interventions to decrease sun exposure in humans in an effort to prevent skin cancer. The reduction of sun exposure during the childhood and adolescent years has been shown to prevent the development of skin cancer in later adulthood (CDC, 2011). Because a large portion of children's lives are spent in a classroom setting, teachers and educational institutions should take advantage of the opportunity to diffuse 
sun safe knowledge throughout the curriculum, providing children with an early advantage in developing precautionary behaviors when taking part in outdoor activities. Guidelines have been developed by the CDC for school programs to prevent skin cancer (CDC, 2011). In developing the guidelines, various collaborations were used to formulate a comprehensive approach to preventing skin cancer among young people using pediatricians, dermatologists, universities, public health organizations, and others at the national, federal and state levels (CDC 2011). As presented, the project is supported by foundational research and national guidelines for skin cancer prevention.

\section{Theoretical Framework Support of Project}

Kotter's (1996) theory of leading organizational change supported the implementation of this project. Each of the 8 stages of change focused on specific actions performed during certain phases of the project. Creating the climate for change within the organization included increasing the sense of urgency, building the guiding the team, and getting the right vision to introduce positive behavior changes regarding sun prevention. Enabling and engaging the organization as a whole occurred through the communication sessions with both participants and administration to obtain buy-in, empowering action, and creating short-term wins. Finally, implementing and sustaining the transformation occurred by continuous reinforcement of the positive behavior to encourage project support. Each of Kotter's (1996) steps were discussed in great detail and lend support to the project in the theoretical framework section of the proposal.

\section{Feasibility Analysis}

The total amount budgeted for the proposed project was $\$ 1,298.73$. The proposed itemized budget is included with this proposal in Appendix A. Approximately $\$ 578.73$ was covered by the participating facility, and the remaining $\$ 365.00$ was provided by the student investigator. Although originally, the proposed project included the availability of classroom 
teachers as an integral part of the intervention, the student conducted the educational interventions to maintain interrater reliability and consistency with content delivery. Forty staff members participated in the educational intervention, including health care professionals, regular facility staff, behavioral health technicians, and therapists working directly with the patient population on both day and evening shifts. Also included in the project planning were the administrative staff consisting of the Medical Director, Chief Executive Officer (CEO), Chief Operating Officer (COO), Chief Financial Officer (CFO), and director of education. Although the intervention from the Sun Smart U Curriculum was intended to be delivered in two 40-50minute sessions, adjustments to staff scheduling and external factors resulted in four 40-50minute sessions delivered to a total of 40 participants. The same content was delivered in each session to different groups of participants, the final number totaling $\mathrm{N}=40$. The participants were paid at the current employee hourly rate including fringe benefits. The investigator provided the SunSmart U curriculum as the intervention at no cost to the facility. Both the curriculum and space for the teaching was provided without additional expense. The investigator agreed to provide the food for all 4 sessions of the lunch and learn activities. There were printing costs for the notebook with the curriculum which has been given to the director of education. Paper handouts, writing supplies, and the pre/posttest were supplied, and the costs covered by the investigator. There were no travel expenses budgeted due to the close proximity of the participating facility. Return on investment will be difficult to estimate, due to the time frame of the proposed intervention and evaluation completion. Some benefits of the intervention are more long-term, and thus will be immeasurable at this time. A revised actual budget for the project is included in Appendix J. 


\section{Resources}

The largest expense for project implementation was the administrative costs for the classroom teachers, hospital staff and employees, and the fringe benefits included therein. The SunSmart U curriculum is designed to be approximately 40 to 50 minutes in length. The sessions remained 40-50 minutes in length; however, the investigator made adjustments to the number of times the content was delivered. The actual educational intervention was given at 4 different times, with 4 separate groups of participants $(\mathrm{N}=40)$. This adjustment was made due to unforeseen circumstances with staff scheduling. Therefore, 40 hospital staff received the 40-50 minutes intervention at a paid rate of $\$ 10.00 /$ hour for a total of $\$ 400.00$. This compensation was provided by the participating facility on the employees' paycheck in addition to the FICA (7.65\%), worker's compensation $(0.54 \%)$, and health insurance $(30 \%)$ benefits totaling $\$ 178.73$. The Skin Cancer Foundation provided the SunSafe U curriculum and posters at no cost; however, the cost of printing the Sun Smart U Curriculum and providing it to the facility in a bound copy was approximately $\$ 25.00$. The cost allowance has been estimated for approximately 40 employees, and fluctuations in staff pay levels were taken into consideration totaling $\$ 578.73$. The salaries of the employees for project implementation were provided in kind from the participating facility; therefore, the direct supplies purchased such as handouts and pretest/posttest forms including demographic information for the actual educational intervention totaled approximately $\$ 20.00$. In order to increase participation, the educational offering was given in a 'lunch and learn' type atmosphere with food provided by the DNP student at approximately $\$ 250.00$. The only recurring cost yearly to the institution would be the general program supplies, should the facility choose to adopt the pilot study results as a permanent system change. 


\section{Congruence of Organizations Strategic Plan to Project}

The proposed project was congruent with the organizational site's strategic plan as evidenced by their mission statement, values, vision, and core purpose. For example, the mission of the facility where the project was conducted focuses on providing quality behavioral health services to children, adolescents, and adults in a caring environment. The intent of this capstone proposal was to facilitate quality care through education and training of patients and staff in sun prevention methods with the goal of increasing use of sunscreen during outdoor recreation for UV protection or sun overexposure. The core purpose of the organization is to improve the health of the people they serve. The overall objective of this project was to improve the overall health of the children at the facility through early intervention with sunscreen education and decrease sun exposure, therefore helping to decrease their risk of developing skin cancer in later adulthood. The organization also supports certain values as stated in the strategic plan such as quality care, patient-centered environment, ethical treatment of all, innovation, fiscal responsibility, total well-being, investment in their people, and visibility.

The capstone project helped staff to provide quality care through offering the resources needed to assist health care providers in making good choices when exposed to the sun's rays during peak hours, and to influence these behaviors with the pediatric population they serve. The project itself was patient-centered because the education was directed to the health care professionals and staff caring for children between the ages of 4 and 14 through a classroom-type setting using a set curriculum from Sun Smart U including interactive lesson plans, videos, pretest post-test quizzes, and self-made posters from the students/residents. Finally, the organization's vision is to be a premier provider of quality behavioral health care services and improve the total well-being of individuals and families through investment in their employees, patients, and communities. This project will invest in the total well-being of both the staff and 
residents of the facility by investing in his or her future providing the education and resources necessary to choose sun safe behaviors involving sunscreen usage during outdoor activity.

The organization follows the regulations set forth by the West Virginia State Code Title 64 “Legislative Rules Division of Health Series 11 Behavioral Health Centers Licensure” which specifies details of consumer services and basic rights while undergoing treatment within the organization (WV Behavioral Health Centers Licensure, 2000). In section 7.12.c of this document, it clearly states that the "onsite staff shall ensure that each resident receive training and practice good habits in personal care, hygiene, and grooming (WV Behavioral Health Centers Licensure, 2000. p. 31). Reinforcing the purpose of this project, the staff was provided with necessary education to ensure the residential children are being afforded the benefits of healthy choices and behaviors that involve sun safety practices and sunscreen usage. Also, in this document provided under section 7.13.a, it states that the behavioral health center will provide programs for minors that are child-centered with integrated educational interventions that "respect the child's developmental process" (WV Behavioral Health Centers Licensure, 2000, p. 32). The Sun Smart U curriculum was appropriate for the population of the institution and its caregivers and was directly tailored to the developmental process of the child.

The strategic plan of the organization where the project was implemented, as well as the West Virginia State Code Title 64 “Legislative Rules Division of Health Series 11 Behavioral Health Centers Licensure” lent evidence and support to the proposed project to provide an intervention for sunscreen protection ensuring the healthy behaviors of institutionalized children. The proposed project was a method to engage the staff and residential children of the facility in lifelong sun safety behaviors that would support the overarching Healthy People 2020 objective C-20 to increase the proportion of persons that participate in behaviors to decrease exposure to harmful ultraviolet irradiation and avoid sunburn (Office of Disease Prevention and Health 
Promotion, 2018). A letter of support from the participating facility has been provided by the CEO, and is presented as Appendix B.

\section{Project Timeline}

The project took place between July $1^{\text {st }}, 2018$ and April 26th, 2019. The project was discussed in great detail with the CEO and administrative team at the participating organization. Written approval was obtained from the organization by July $9^{\text {th }}$, 2018. After written approval was obtained from the participating facility's CEO and DNP Capstone Committee, formal application to the WVU Institutional Review Board (IRB) began. Only after IRB authorization did the on-site educational intervention take place.

The Sun Smart U curriculum and project implementation details were discussed with administration between November $1^{\text {st }}, 2018$ and November $20^{\text {th }}, 2018$. These specially scheduled sessions gave facility administration the opportunity to ask questions regarding project implementation, and detailed information regarding confidentiality and security methods used to protect the facility and study participants. The DNP student worked with the Director of Nursing and Patient Care Services and Director of Training and Organizational Development to schedule all participating employees for the educational sessions, allowing for make-up classes when applicable. After all requirements had been respectfully met for the IRB, materials for Sun Smart U curriculum were printed and placed in the facility to initiate system change. The actual intervention was fully implemented between December $15^{\text {th }}, 2018$ and January 17, 2019. A complete debriefing will be provided to the administration at the participating facility detailing the findings and conclusions from the project.

Sustainability of the Sun Smart U curriculum and knowledge gained from the proposed project was the primary goal of this intervention. However, in order to fully support this, the DNP student explored the possibility for adding sunscreen to the pharmacy formulary. This 
included reviewing current policies at the organization and seeking evidence-based literature supporting sun prevention/skin protection during outdoor activities involving the children and adolescents. Adding sunscreen to the pharmacy formulary and the development of an outdoor activities policy was not feasible for the time frame of this project; however, these possibilities can be explored for future development of sun prevention activities within this facility. A Gantt chart is provided in Appendix C outlining the timeframe for the project.

\section{Measurable Project Objectives}

There were four measurable project objectives. First, the staff's level of knowledge regarding sun preventive behaviors that were measured using the Sun Smart U Curriculum truefalse questionnaire from the Skin Cancer Foundation both (1) before and (2) after the SunSafe U intervention (Appendix D). Then, (3) the staff's intent to change behavior was measured post intervention using the Continuing Professional Development (CPD) Reaction Questionnaire. Finally, (4) the feasibility of implementing behavior change within the organization was evaluated. This tool was developed by Légaré et al. (2017) in an attempt to measure desired behavior, change for quality improvement. The CPD-R tool is valid and reliable (Cronbach's coefficients for constructs varied from 0.77 to 0.85 ) for assessing the impact of Continued Professional Development activities on the behavioral intentions of healthcare providers (Légaré et al., 2014). An email communication granting the permission to use the tool for the purposes of this capstone is provided in Appendix E. A modified version of this tool is provided in Appendix F for the purpose of capturing participant intent to change behavior post intervention. Finally, (4) a short 3- question survey was given post intervention to identify the feasibly of the intent to change personal behavior as applied to daily practice (Appendix G). Demographic data was collected for the purpose of reporting descriptive statistics of the participants (Appendix H). 
See Table 1 below for summarization of data collection methodology, measurable goals, expected outcomes, instruments, and statistical tests used for the current study.

Table 1

Measurable Goals and Outcomes

\begin{tabular}{|c|c|c|c|c|}
\hline Methods & Goals & Expected Outcomes & Instrument & Statistical Test \\
\hline $\begin{array}{l}\text { Pre-test } \\
\text { Sun Smart U }\end{array}$ & $\begin{array}{l}\text { Demonstrate } \\
\text { baseline level } \\
\text { of knowledge }\end{array}$ & $\begin{array}{l}\text { Low level of knowledge } \\
\leq 80 \%(8 / 10)\end{array}$ & $\begin{array}{l}\text { Sun Smart U } \\
\text { Pre-test }\end{array}$ & $\begin{array}{l}\text { Parametric } \\
\text { Summary } \\
\text { Statistics, } \\
\text { Paired t-test }\end{array}$ \\
\hline $\begin{array}{l}\text { Post-test } \\
\text { Sun Smart U }\end{array}$ & $\begin{array}{l}\text { Demonstrate } \\
\text { increased level } \\
\text { of knowledge }\end{array}$ & $\begin{array}{l}\text { High level of knowledge } \\
\geq 80 \%(8 / 10)\end{array}$ & $\begin{array}{l}\text { Sun Smart U } \\
\text { Post-test }\end{array}$ & $\begin{array}{l}\text { Parametric } \\
\text { Summary } \\
\text { Statistics, } \\
\text { Paired t-test }\end{array}$ \\
\hline $\begin{array}{l}\text { Post-test } \\
\text { CPD-R }\end{array}$ & $\begin{array}{l}\text { Measure level } \\
\text { of intent to } \\
\text { change behavior }\end{array}$ & $\begin{array}{l}\text { Personal intention } \\
\text { to change behavior }\end{array}$ & CPD-R & $\begin{array}{l}\text { Parametric } \\
\text { Summary } \\
\text { Statistics, } \\
\text { Spider-plot }\end{array}$ \\
\hline $\begin{array}{l}\text { Demographic } \\
\text { Data }\end{array}$ & $\begin{array}{l}\text { Collect } \\
\text { descriptive data } \\
\text { for reporting } \\
\text { purposes }\end{array}$ & $\begin{array}{l}\text { General characteristics } \\
\text { of convenience sample } \\
(\mathrm{N}=40)\end{array}$ & Survey & $\begin{array}{l}\text { Parametric } \\
\text { Descriptive } \\
\text { Statistics }\end{array}$ \\
\hline $\begin{array}{l}\text { Feasibility } \\
\text { Survey }\end{array}$ & $\begin{array}{l}\text { To predict } \\
\text { feasibility of } \\
\text { behavior change } \\
\text { implementation } \\
\text { in facility }\end{array}$ & $\begin{array}{l}100 \% \text { feasibility } \\
(3 / 3)\end{array}$ & Survey & $\begin{array}{l}\text { Parametric } \\
\text { Descriptive } \\
\text { Statistics }\end{array}$ \\
\hline
\end{tabular}

\section{Project Description and Design}

The project itself was instituted using the teacher resources from Sun Smart U entitled “Rays Awareness - Preventing Skin Cancer" (Skin Cancer Foundation, 2018). The residential facility staff was presented with the lesson plan and activities from the Sun Smart U information in classrooms provided by the participating organization. The original Sun Smart U Curriculum 
is intended to be delivered in two 40-50-minute session to a participating audience. In order to accommodate unforeseen staff scheduling changes, the student delivered the educational intervention in four 40-50 -minute sessions to accommodate the study participants. The consistencies of the same student presenter and same educational intervention were evident for reporting purposes. Only the DNP student delivered the Sun Smart U content to the participants directly to assist with consistency and control for extraneous variables. This strategy was taken so the material was given in a familiar environment, maintain consistency with content delivery, and to increase participant comfort level and participation with the project.

\section{Data Collection}

The project was conducted using a Pre-Posttest design with a convenience sample of 40 participants from the consenting health care facility. Of notable mention for content delivery, the Skin Cancer Foundation recommends two class periods of forty minutes each; however, due to the time constraints and scheduling of all participating employees, the sessions had to be divided into 4 contact interventions lasting approximately 40 minutes each. Each participant was given a packet of coded materials ranging from $\# 001$ to $\# 040$ for data collection and eventual statistical comparison and data analytics. The lesson to be delivered was aligned to the CDC National Health Education Standards (NEHS) for grades 6-12 (Skin Cancer Foundation, 2018), therefore targeting the special population of the facility. Learning objectives for the staff included: understanding ultraviolet radiation including both UVA and UVB rays; explaining how UV radiation affects the skin; understanding the effects of indoor and outdoor tanning; understanding the various forms of skin cancer; understanding how to detect skin cancer; and protecting themselves from UV radiate on exposure. The lesson proceeded with video segments including real stories from young adult skin cancer survivors, the ABCDE's of melanoma, the ugly duckling signs, other forms of precancer, a Sun Smart Steps handout and animated video, and 
finally how to go with your own glow. A copy of the Sun Smart U curriculum has been provided as Appendix I.

\section{Inclusion and Exclusion Criteria}

Participant inclusion criteria included adults aged 18 and over, current employment status at the health care facility where the educational intervention took place, and involvement in direct patient care. All subjects were given a letter of consent explaining the study, and the completion of the educational intervention served as his or her intent to fully participate.

Exclusion criteria included any person under the age of 18 years, not employed at the participating facility, and not involved in the direct care of the target population.

\section{Project Evaluation Plan and Measurement Instruments}

As depicted in the Table 1, there were four specific outcomes measured with the implementation of this project: (1) the staff's baseline level of knowledge regarding sun protective behaviors, (2) the staff's level of knowledge regarding sun protective behaviors post educational intervention with Sun Smart U Curriculum, (3) the staff's intent to change behavior; and, (4) the feasibility of implementing behavior change within the organization. Both the outcome evaluation and process evaluation were used to determine the effectiveness of the capstone project. The statistical software utilized in data analysis was JASP (Version 0.9.2, 2018) which is an open-source graphical program designed for familiar users of SPSS.

According to the statistician assisting with data analysis, this is the desired program to accurately describe both visual and numerical leveled outcomes.

Statistical analysis was performed initially using parametric summary statistics and a paired t-test for comparison of Pre and Posttest mean scores examining differences among group means in the chosen sample. This analysis provides an indication of an increase or decrease in level of knowledge of sun protective and prevention behaviors. Secondly, parametric summary 
statistics and a spider plot was used on the CPD-R questionnaire results to measure the level of intent of participants to change behavior. Results illustrated in the spider plot display which of the 5 constructs (e.g. intention, social influence, beliefs about capabilities, moral norm, and beliefs about consequences) have the highest impact on the participants' ability to change their behaviors. Parametric descriptive statistics were also used to report participant demographic data. Finally, a Chi-square test for independence was explored to determine an association between two categorical variables; in this case, the variables would be sun protection usage and age, gender, or education. This data is explained in contingency tables of the results section.

\section{Results}

\section{Objectives 1 and 2}

The first objective was to examine the level of knowledge using a Pre-test from the Sun Smart U curriculum relating to sun prevention and sunscreen usage prior to the classroom educational intervention. This True-False questionnaire was given prior to the start of the planned educational intervention. According to the previously mentioned expected outcomes in Table 1, the participants were expected to have a lower level of knowledge regarding sun preventive behaviors. The second measurable objective was to examine the level of knowledge using a posttest from the Sun Smart U curriculum relating to sun prevention and sunscreen usage post classroom educational intervention. To be consistent, the same Sun Smart U pretest using the True-False questionnaire was administered after the educational intervention, and scores were compared using the paired t-test with results also depicted in Table 2 . To perform the paired t-test, participant \#026 was eliminated specifically because of the incomplete posttest. Therefore, the participant number for this analysis is $\mathrm{N}=39$. Table 2 represents the summary statistics for both pre and posttest data, as well as the differences in scores and the percentages of calculated change. 
On the pretest scores, the average was $\sim 93 \%$ (93.08) compared to the average score on the posttest of $97.68 \%$. This indicates that there was a $5.897 \%$ change in the mean scores from pretest to posttest in an upward direction. Prior to the educational intervention, the content expert on this capstone committee recommended that a threshold of $80 \%$ (8/10 correctly answered questions) was considered knowledgeable and depicted literacy on the topic of sun prevention behaviors (G. Daniel, personal communication, January 23, 2019). The percent change and the average is $\sim 6 \%$ with a wide range of variation; some participant's scores were as low as $22 \%$ less on his or her posttest compared to the same individual's pretest. However, some participants scored up to $43 \%$ more on his or her posttest compared to the same individual's pretest. Given the fact that the participants in this intervention were employed at a medical facility and provided both direct and indirect care for the patient population, an approximate pretest score of $93 \%$ was acceptable.

Table 2

Descriptive Statistics Pretest and Posttest Scores and Percentage Change on Exam Scores $(n=39)$

\begin{tabular}{lcccc}
\hline & PreTest & PostTest & Difference & \%Change \\
\hline Mean & 93.08 & 97.69 & 4.615 & 5.897 \\
SEM & 1.430 & 1.069 & 1.595 & 2.061 \\
Std. Deviation & 8.931 & 6.673 & 9.959 & 12.87 \\
Skewness & -1.134 & -3.062 & 0.7855 & 1.295 \\
Kurtosis & 0.4428 & 9.055 & 1.397 & 2.415 \\
& & & & \\
\hline
\end{tabular}

Also examined were the skewness, kurtosis, and histogram depiction to describe and illustrate the distribution of the variables. Although both the pre (-1.134) and posttest $(-3.062)$ scores are skewed to the left (pre-scores less so than post scores), the distribution of the difference between the scores is not skewed. A value less than $|1|$ can be defined as 
approximately normal. This is important because the paired t-test assumes that the differences between pairs are normally distributed. If the differences between pairs would have been severely abnormal, it would have been better to use the non-parametric Wilcoxon signed-rank test, but because this data set met the assumptions for parametric testing, the parametric test was used instead. In addition, the paired t-test does not assume that the groups are homoscedastic. The Histograms depicting the differences are represented in Figure 1.

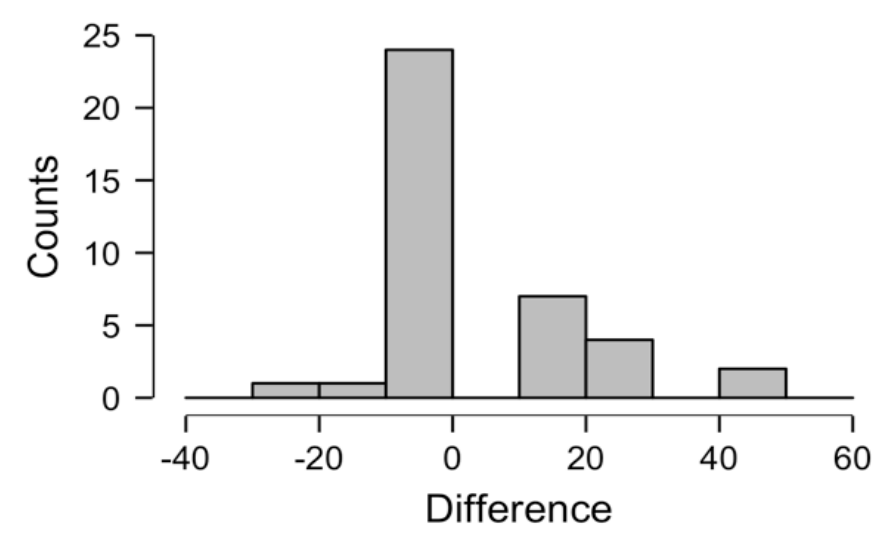

Figure 1. Distribution of the differences between the mean pretest and posttest scores The null hypothesis used in this objective is that the mean difference between paired observations is zero. If the mean difference between paired observations is zero, this indicates that the means are the same for both groups (e.g. that the educational intervention had no effect in changing the means). The alternative hypothesis used was a 2-tail (non-directional) hypothesis indicating a difference between the two groups, both pretest and posttest. The pvalue of 0.006 shows that the null hypothesis can be rejected with confidence since $\alpha=0.05$, and the data suggests that the means are not equal. In fact, the mean of the scores for the posttest groups (4.615) increased after the skin cancer prevention educational intervention. The 95\% Confidence Interval (CI) for the parameter estimate does not include 0, which is positive. 
Conclusively, it can be deduced with $95 \%$ confidence that the posttest groups scored as low as $1.3 \%$ and as high as $7.8 \%$ more than the pretest groups. Using Cohen's $\mathrm{d}$, the author can conclude there is evidence to suggest there was a statistically significant increase in test scores after the skin cancer prevention educational intervention. The results are depicted in Table 3 below.

Table 3

Depiction of Paired Samples T-Test

Paired Samples T-Test

\begin{tabular}{|c|c|c|c|c|c|c|c|c|c|}
\hline & & \multirow[b]{2}{*}{$\mathrm{t}$} & \multirow[b]{2}{*}{$\mathrm{p}$} & \multirow[b]{2}{*}{$\begin{array}{c}\text { Mean } \\
\text { Difference }\end{array}$} & \multirow[b]{2}{*}{$\begin{array}{c}\text { SE } \\
\text { Difference }\end{array}$} & \multicolumn{2}{|c|}{$\begin{array}{l}95 \% \text { CI for } \\
\text { Mean } \\
\text { Difference }\end{array}$} & \multirow[b]{2}{*}{$\begin{array}{c}\text { Cohen's } \\
\mathrm{d}\end{array}$} & \multirow{2}{*}{$\begin{array}{c}\begin{array}{c}95 \% \text { CI for } \\
\text { Cohen's d }\end{array} \\
\text { Lower Upper }\end{array}$} \\
\hline & & & & & & Lower & Upper & & \\
\hline PostTest & - PreTest & 2.89438 & 0.006 & 4.615 & 1.595 & 1.387 & 7.844 & 0.463 & $0.130 \quad 0.791$ \\
\hline
\end{tabular}

Note. Student's t-test.

Cohen suggested that $d=0.2$ be considered $a$ 'small' effect size, 0.5 represents $a$ 'medium' effect size and 0.8 a 'large' effect size.

\section{Objective 3}

The third measurable objective was to examine the participant's level of intent to change behaviors incorporating sun prevention into daily practice after the educational intervention had been completed. The Continuing Professional Development Reaction (CPD-R) tool was utilized in measuring the level of the health care professional's intent of translating the proposed behavioral changes into daily practice. Permission to use the tool (Appendix E) was obtained from the original author, France Légaré, and an example of the adapted tool is depicted in Appendix F. The tool shows adequate validity and reliability with Cronbach's coefficients for 
the various constructs ranging from 0.77 to 0.85 (Légaré et al., 2017). This instrument consists of 12 items assimilating social and cognitive theories that attempt to explain behaviors of the health professional via intention (examples include the Theory of Planned Behavior and Triandis' theory) (Légaré et al., 2017). In Légaré et al. (2017), three categorical variables predict behavior: (1) intention to adopt to a particular behavior; (2) beliefs about capabilities; and (3) past habits and behavior. The 5 constructs measured include intention, social influence, beliefs about capabilities, moral norms, and beliefs about consequences (Légaré et al., 2017). A summary of the CPD-Reaction questionnaire scores on items and constructs of behavioral measurement as used to analyze data in the present study are depicted in Figure 2 below.

\begin{tabular}{|c|c|c|c|c|c|c|}
\hline Construct scale & & Items $\mathbf{s}^{\mathrm{a}}$ & $\begin{array}{l}\text { Responses } \\
\text { choices }\end{array}$ & $\begin{array}{l}\text { Pre-coded } \\
\text { item value }^{b}\end{array}$ & $\begin{array}{l}\text { Final item } \\
\text { score }^{c}\end{array}$ & $\begin{array}{l}\text { Score by } \\
\text { construct }^{d}\end{array}$ \\
\hline \multirow[t]{2}{*}{ Intention } & $l_{1}$ & I intend to [behavior] & $\begin{array}{c}\text { Strongly } \\
\text { disagree/agree }\end{array}$ & 1 to 7 & 1 to 7 & \multirow[t]{2}{*}{$\left(I_{1}+I_{7}\right) / 2$} \\
\hline & $\mathrm{I}_{7}$ & I plan to [behavior] & $\begin{array}{c}\text { Strongly } \\
\text { disagree/agree }\end{array}$ & 1 to 7 & 1 to 7 & \\
\hline \multirow[t]{7}{*}{ Social influence } & \multirow[t]{5}{*}{$\mathrm{I}_{2}$} & \multirow[t]{5}{*}{$\begin{array}{l}\text { To the best of my knowledge, the percentage of my } \\
\text { colleagues who [behavior] is... }\end{array}$} & $0-20 \%$ & 1 & 1.4 & \multirow[t]{7}{*}{$\left(I_{2}+l_{6}+l_{9}\right) / 3$} \\
\hline & & & $21-40 \%$ & 2 & 2.8 & \\
\hline & & & $41-60 \%$ & 3 & 4.2 & \\
\hline & & & $61-80 \%$ & 4 & 5.6 & \\
\hline & & & $81-100 \%$ & 5 & 7 & \\
\hline & $I_{6}$ & $\begin{array}{l}\text { Now think about a co-worker whom you respect as a } \\
\text { professional. In your opinion, does he/she [behavior]? }\end{array}$ & Never/Always & 1 to 7 & 1 to 7 & \\
\hline & $I_{9}$ & $\begin{array}{l}\text { Most people who are important to me in my profession } \\
\text { [behavior] }\end{array}$ & $\begin{array}{c}\text { Strongly } \\
\text { disagree/agree }\end{array}$ & 1 to 7 & 1 to 7 & \\
\hline \multirow[t]{3}{*}{$\begin{array}{l}\text { Beliefs about } \\
\text { capabilities }\end{array}$} & $I_{3}$ & I am confident that I could [behavior] if I wanted to. & $\begin{array}{c}\text { Strongly } \\
\text { disagree/agree }\end{array}$ & 1 to 7 & 1 to 7 & \multirow[t]{3}{*}{$\left(l_{3}+l_{5}+l_{11}\right) / 3$} \\
\hline & $\mathrm{I}_{5}$ & For me, [behavior] would be. . . & $\begin{array}{l}\text { Extremely } \\
\text { difficult/easy }\end{array}$ & 1 to 7 & 1 to 7 & \\
\hline & $I_{11}$ & I have the ability to [behavior] & $\begin{array}{c}\text { Strongly } \\
\text { disagree/agree }\end{array}$ & 1 to 7 & 1 to 7 & \\
\hline \multirow[t]{2}{*}{ Moral norm } & $\mathrm{I}_{4}$ & [Behavior] is the ethical thing to do. & $\begin{array}{c}\text { Strongly } \\
\text { disagree/agree }\end{array}$ & 1 to 7 & 1 to 7 & \multirow[t]{2}{*}{$\left(l_{4}+l_{10}\right) / 2$} \\
\hline & $I_{10}$ & It is acceptable to [behavior] & $\begin{array}{c}\text { Strongly } \\
\text { disagree/agree }\end{array}$ & 1 to 7 & 1 to 7 & \\
\hline \multirow{2}{*}{$\begin{array}{l}\text { Beliefs about } \\
\text { consequences }\end{array}$} & $I_{8}$ & Overall, I think that for me [behavior] would be... & Useless/Useful & 1 to 7 & 1 to 7 & \multirow[t]{2}{*}{$\left(l_{8}+l_{12}\right) / 2$} \\
\hline & $I_{12}$ & Overall, I think that for me [behavior] would be... & $\begin{array}{l}\text { Harmful/ } \\
\text { Beneficial }\end{array}$ & 1 to 7 & 1 to 7 & \\
\hline
\end{tabular}

\footnotetext{
a Item number (e.g., $I_{1}=$ Item 1)
}

${ }^{b}$ Pre-coded item value is a Likert scale assigned value (i.e., Strongly disagree $=1$, Strongly agree $=7$; Never $=1$, Always $=7$, etc.)

${ }^{\circ}$ Final item score is the score by item for each participant (possible range scale $=1$ to 7 )

${ }^{\mathrm{d}}$ Score by construct $=$ mean score by construct (possible range scale $=1$ to 7 ).

Note: for constructs with two items, no imputed values are possible. For constructs with three items, the raw score of the scale is missing if two or more items are missing. In the case of one missing item, the missing item is imputed from the mean of the two other item. 
Figure 2. Summary of CPD-Reaction questionnaire scores on items and 5-constructs (Légaré et al., 2017, p. 4)

The CPD-Reaction survey results illustrate the average scores for all participants on the 5 constructs of intention, social influence, beliefs about capabilities, moral norms, and beliefs about consequences $(\mathrm{N}=40)$. The Standard Error of the Mean (SEM) is an estimate of the standard deviation of the population, and the SEMs are depicted as the slide bar on top of the columns in Figure 5. For Intention, the mean score was 5.0 with an SEM of 0.25 and standard deviation (SD) of 1.6. The results for the Social Influence construct show a mean of 4.4, SEM of 0.19, and SD of 1.2. Beliefs about capabilities show a mean of 4.9, SEM of 0.11, and SD of 0.7. the Moral Norm construct show a mean of 4.8, SEM of 0.25 , with a SD of 1.6. The final construct of Beliefs About Consequences shows a mean of 6.4, SED of 0.21, and SD of 1.3. Based on the results of this analysis, the author concludes that the most positive social construct most likely to affect sun protective behavior change with the population under study is Beliefs About Consequences. Supporting this conclusion, literature from Légaré et al. (2017) suggested that the CPD-Reaction questionnaire is "responsive enough to detect a change in the behavioral intention of health care professionals attending CPD activities", and "the integrated conceptual model upon which the CPD-Reaction is based proposed that behavioral intention is a predictor of behavior" (p. 10). Hence, the higher mean score of 6.4 for Beliefs About Consequences indicate that the participants' behavior change (if measured again in the future) would most likely be due to his or her beliefs about the consequences of not participating in sun prevention behaviors (e.g. sunscreen, protective clothing, seeking shade, etc.). Figure 3 below depicts the CPD-Reaction survey results. Figure 4 displays the results of the 5 constructs in a Spider plot format for visual illustration. The spider plot (as mirroring the results of the bar graph) depicts, on average, Beliefs About Consequences is the construct with the highest impact on the participants in this study, 
and his or her ability to change behaviors followed by Beliefs About Capabilities and Intention.

The least influential were the Social Influence and Moral Norm constructs.

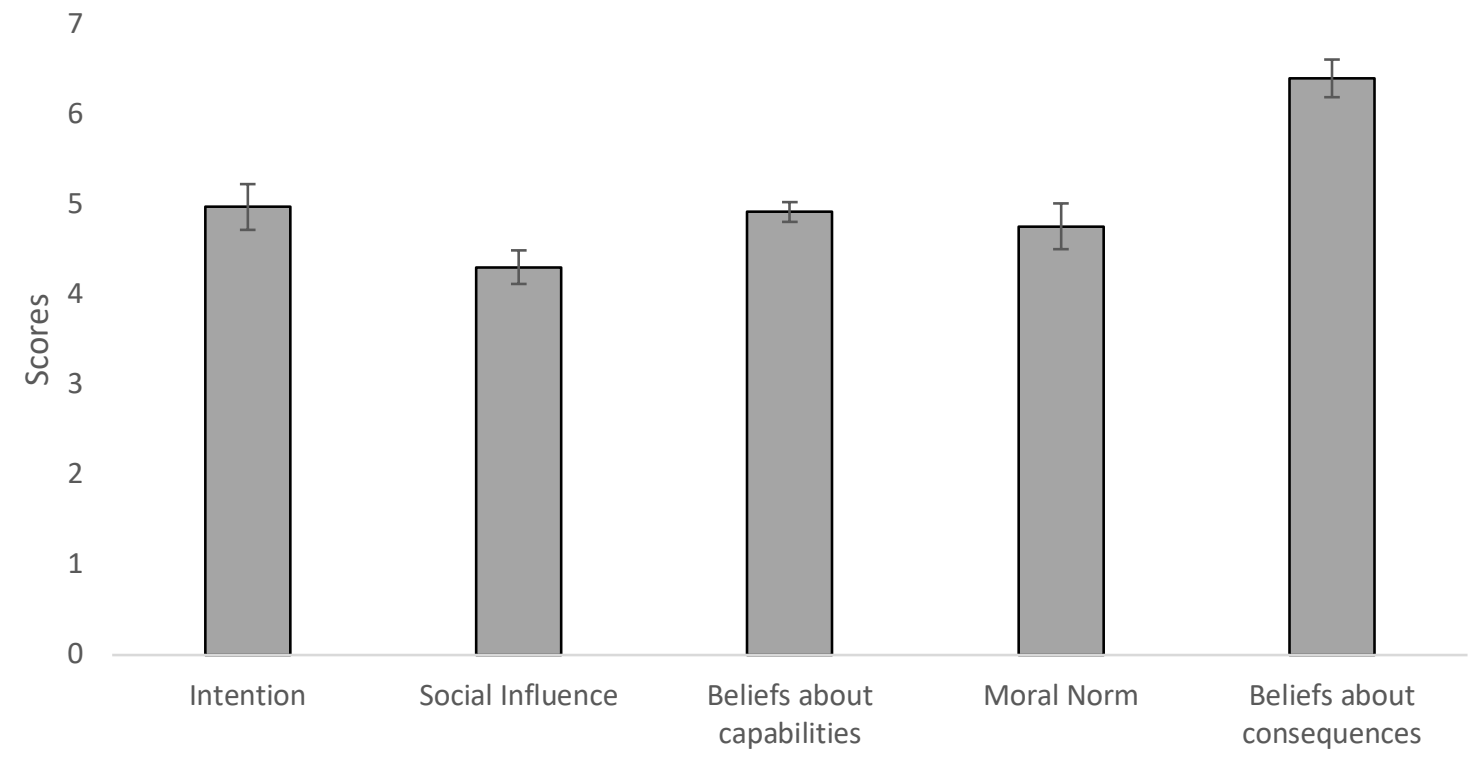

Figure 3. Average CPD-Reaction scores for all participants on each construct $(\mathrm{N}=40)$.

Standard error bars shown. Scores presented are on a Likert scale (0-7). 


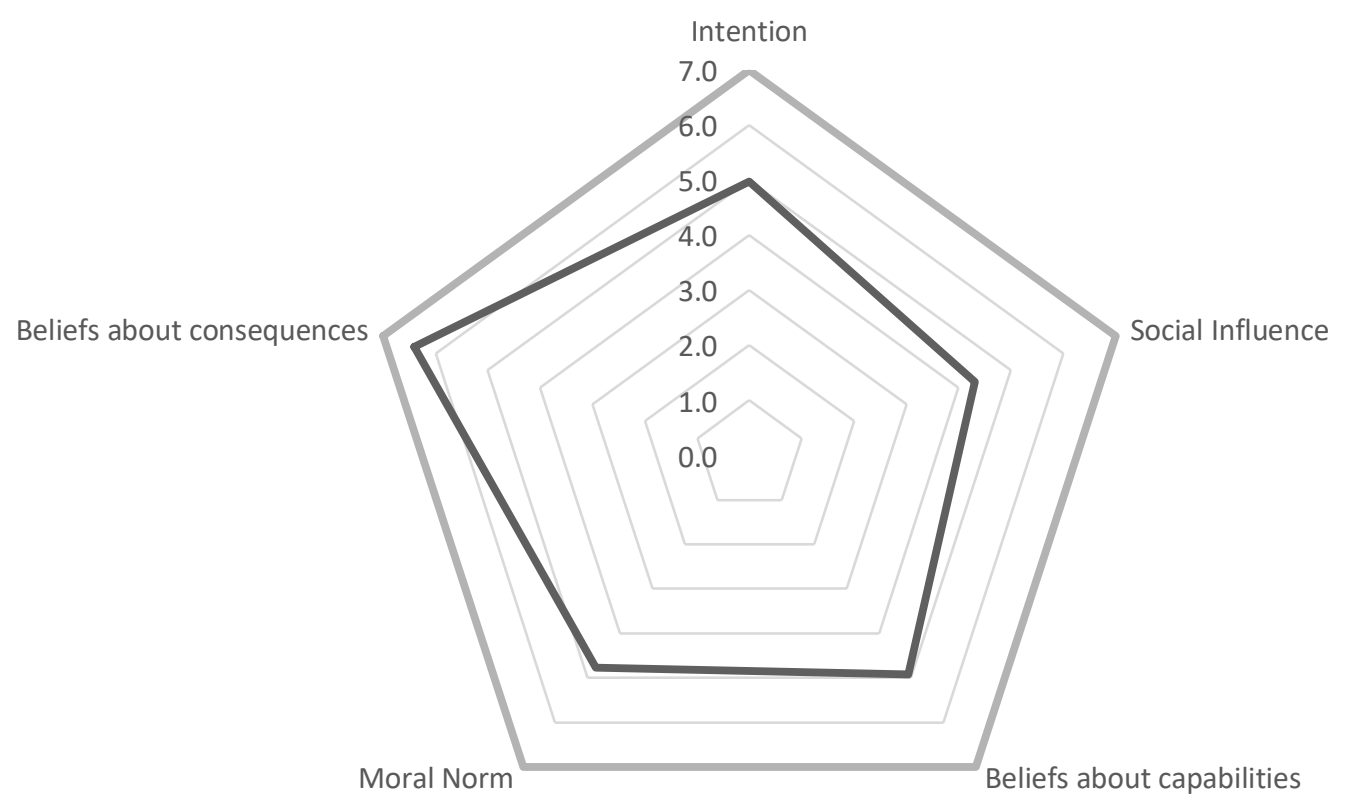

Figure 4. Spider plot of all 5 constructs measured by the CPD-R tool questionnaire $(\mathrm{N}=40)$. Maximum scores are shown in bolded grey.

\section{Objective 4}

The fourth objective was to collect general demographic information to reveal the characteristics of the participant population and make inferences based on the anonymous data. There was a total of $\mathrm{N}=40$ participants in the study (female $=37$, male, 3 ). Fifteen of the participants had at least a Bachelor's level of education $(n=15)$ while the majority combined $(n=18)$ had a Master's Degree $(n=6)$, Associate's Degree $(n=6)$, and some college $(n=6)$. Only 7 participants had only completed high school $(n=5)$ or a vocational education $(n=2)$. In essence, the majority of the participants $(68 \%)$ had completed some level of college education, $20 \%$ had some college or vocational level education, and $12 \%$ had a high school diploma. From these data, the author can conclude the majority of the target population had some level of higher 
education completion. Both gender and educational levels are illustrated in Figures 5 and 6.

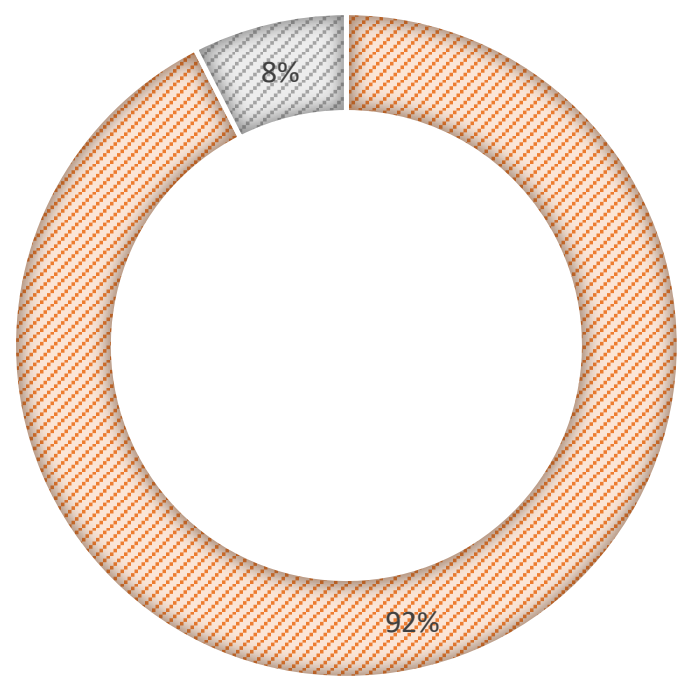

- Gender

a

- $\mathrm{M}$

Figure 5. Patient demographic information displaying gender

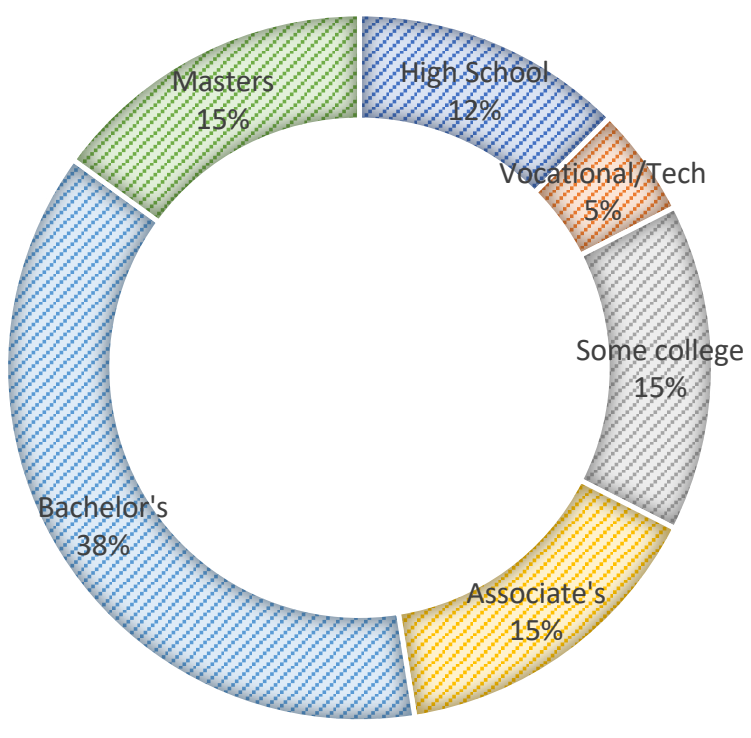

- High School

nocational/Tech

- Some college

- Associate's

- Bachelor's

Masters 
Figure 6. Patient demographic information displaying educational level

When asked what kind of preventive measurement the participants used to protect themselves from the sun, only $40 \%$ claimed to use both sunscreen $(n=16)$ and protective clothing $(n=4)$. A combined 50\% (both sunscreen and protective clothing) claimed to use one or the other, with the majority relying on sunscreen alone $(n=16)$. Only $10 \%$ of the participants listed that they did not use any kind of protection whatsoever $(n=4)$, while 1 participant left this question unanswered. From this data, inferences can be made that the majority of the participants preferred to use some form of sun protection, whether it was sunscreen application or protective clothing, during outdoor exposure time to prevent skin damage and/or skin cancer. Preferred methods of sun protection are depicted in Figure 7. 


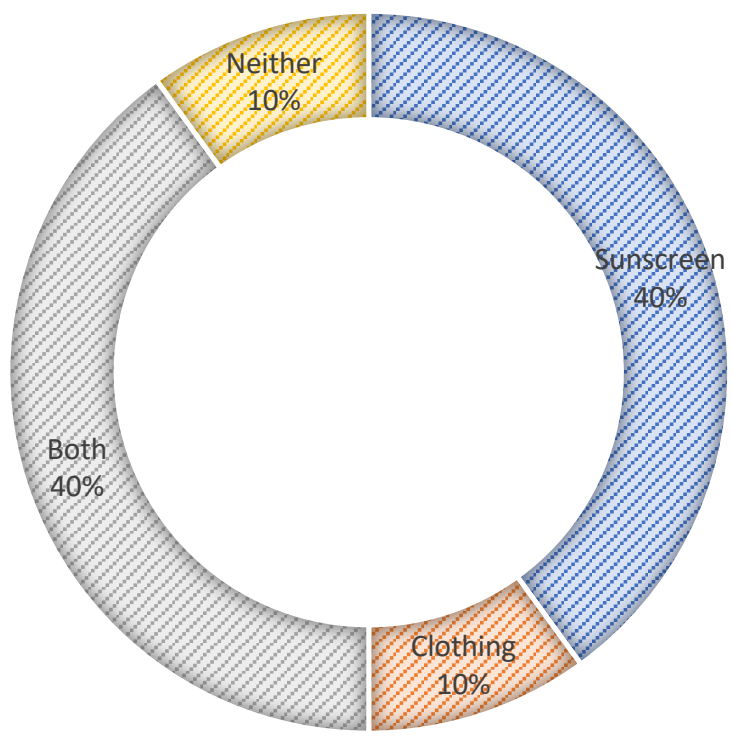

- Sunscreen

- Clothing

a Both

neither

Figure 7. Patient demographic information displaying preferred sun protection

The demographic information also included the participants' ages at the time of the educational intervention. For reporting purposes, the age ranges were categorized in 5 age groupings: $18-25$ years $(n=5), 26-35$ years $(n=11), 36-45$ years $(n=7), 46-55$ years $(n=9)$, and 56 years and over $(n=8)$. The ages are somewhat evenly distributed in 4 of the 5 age groupings. The lowest percentage of the participants included the $18-25$-year-old group (12\%), while the largest percentage included the $26-35$-year-old category (27\%). The distribution of participants' age ranges can be seen in Figure 8. 


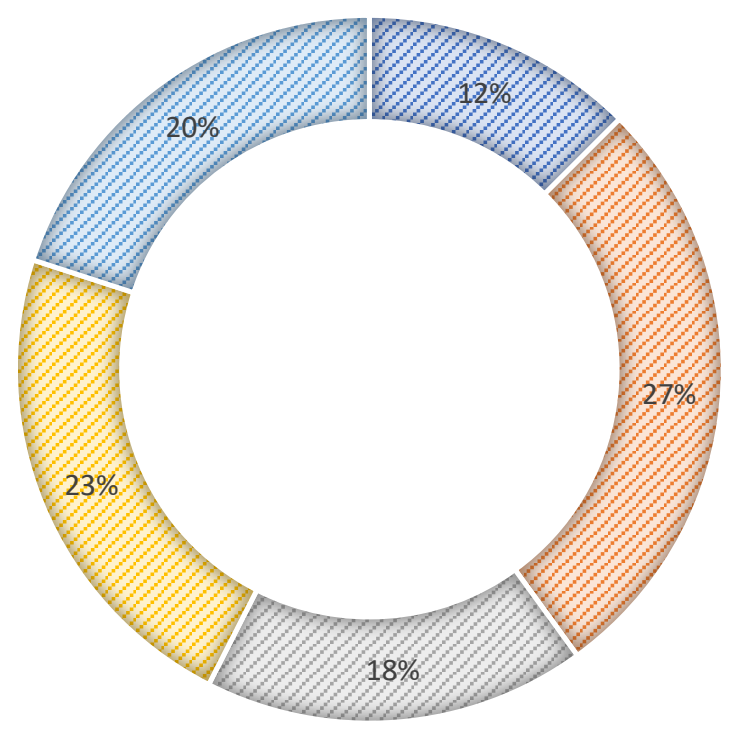

- 18-25

- 26-35

- 36-45

a6-55

56 and over

Figure 8. Patient demographic information illustrating age at time of survey

Finally, participant exposure was examined as part of the demographic survey. The median time out per day for participants was 2 hours with some participants claiming less than 15 minutes per day, and some as long as 12 hours per day. The median time for sun exposure per day was 1 hour and 15 minutes, with a range from 0 hours per day to 10 hours per day. Both the hours out per day and hours out in the sun per day were broken down into percentile categories: $25^{\text {th }}$ percentile, $50^{\text {th }}$ percentile, and $75^{\text {th }}$ percentile. Participants in the $25^{\text {th }}$ percentile spent an average of $1 \frac{1}{2}$ hours per day in the sun and were exposed to less than 1 hour of sun per day. Those in the $50^{\text {th }}$ percentile spent approximately 2 hours out per day in the sun with 1 and $1 / 4$ hours of direct sun exposure per day. Participants in the $75^{\text {th }}$ percentile spent 3 hours per day in the sun with 3 direct hours of sun exposure per day. These results are depicted in Table 4 below. 
Table 4

Descriptive Statistics for Sun Exposure

\begin{tabular}{lll} 
& Hours Out/Day & Hours Sun/Day \\
\hline Median & 2.000 & 1.250 \\
Range & 11.75 & 10.00 \\
Minimum & 0.2500 & 0.000 \\
Maximum & 12.00 & 10.00 \\
$25^{\text {th }}$ Percentile & 1.500 & 0.7500 \\
$50^{\text {th }}$ Percentile & 2.000 & 1.250 \\
$75^{\text {th }}$ Percentile & 3.000 & 3.000 \\
\hline
\end{tabular}

In order to determine if there is a relationship between two categorical variables, in this case protection usage and age, gender, or education, the Chi-square test for independence was used to determine association. The assumptions of the test are twofold: (1) variables are ordinal or nominal; and, (2) variables should consist of two or more categorical, independent groups. The null hypothesis is that there is no association (e.g. the effects on one categorical variable are independent on the other categorical variable). Contingency tables and Chi-square tests were conducted to test the association between sun protection usage and other demographic factors, the first being age and sun protection usage. The Chi-squared value $\left(\mathrm{x}^{2}\right)$ for this tested association was $12.5, d f 16$, and $\mathrm{p}=0.733$, indicating no significant relationship between age and sun protection usage. These results are shown in Table 5. 
Table 5

Contingency Table Testing Association Between Age and Sun Protection Usage

\begin{tabular}{|c|c|c|c|c|c|c|c|}
\hline \multirow[b]{2}{*}{ Age } & & \multicolumn{5}{|l|}{ Protection } & \multirow[b]{2}{*}{ Total } \\
\hline & & sunscreen & protective clothing & both & none & $\mathrm{n} / \mathrm{a}$ & \\
\hline $18-25$ & & 3 & 0 & 2 & 0 & 0 & 5 \\
\hline $26-35$ & & 3 & 2 & 4 & 2 & 0 & 11 \\
\hline $36-45$ & & 4 & 1 & 2 & 0 & 0 & 7 \\
\hline $46-55$ & & 2 & 0 & 4 & 1 & 1 & 8 \\
\hline $56-0 \mathrm{ver}$ & & 4 & 1 & 4 & 0 & 0 & 9 \\
\hline Total & & 16 & 4 & 16 & 3 & 1 & 40 \\
\hline \multicolumn{4}{|c|}{ Chi-Squared Tests } & & & & \\
\hline & Value & \multicolumn{2}{|c|}{$\mathrm{p}<0.05$} & & & & \\
\hline$\overline{X^{2}}$ & 12.15 & \multirow{2}{*}{\multicolumn{2}{|c|}{$\begin{array}{ll}16 & 0.733\end{array}$}} & & & & \\
\hline $\mathrm{N}$ & 40 & & & & & & \\
\hline
\end{tabular}

No statistical significance between Age and Sun Protection Usage 
Contingency tables and Chi-square tests were conducted to test the association between sun protection and other demographic factors, the second being gender and sun protection usage. The Chi-squared value $\left(\mathrm{x}^{2}\right)$ for this tested association was $2.162, d f 4$, and $\mathrm{p}=0.706$, indicating no relationship between gender and sun protection. These results are shown in Table 6 .

Table 6

Contingency Table Testing Association Between Gender and Sun Protection Usage

\begin{tabular}{lllllll} 
& Protection & & & & & \\
\cline { 2 - 6 } Gender & sunscreen & protective clothing & both & none & n/a & Total \\
\hline F & 15 & 3 & 15 & 3 & 1 & 37 \\
M & 1 & 1 & 1 & 0 & 0 & 3 \\
Total & 16 & 4 & 16 & 3 & 1 & 40 \\
\hline
\end{tabular}

Chi-Squared Tests

\begin{tabular}{llll}
\hline & Value & df & $\mathrm{p}<0.05$ \\
\hline $\mathrm{X}^{2}$ & 2.162 & 4 & 0.706
\end{tabular}

$\mathrm{N} \quad 40$

$\overline{\text { No statistical significance between Gender and Sun Protection }}$

Third, a Chi-square test was conducted to test the association between sun protection usage and level of education of the participants. The Chi-square value $\left(\mathrm{x}^{2}\right)$ for this test was 35.50, $d f 20$, and $\mathrm{p}=0.018$, indicating a statistically significant association between the variables. This was the only significant association found. People who had completed higher educational degrees, such as the Associate's, Bachelor's, and Master's, also engaged in more sunscreen or both sunscreen and protective clothing compared to participants with no completed higher educational degrees (e.g. High School, Vocational/Technical School, etc.). These results are illustrated in Table 7. 
Table 7

Contingency Table Testing Association Between Level of Education and Sun Protection Usage

\begin{tabular}{lllllll}
\hline & Protection & & & & \\
\cline { 2 - 5 } Education & sunscreen & protective clothing & both & none & n/a & Total \\
\hline High School & 1 & 2 & 2 & 0 & 0 & 5 \\
Vocational or technical school & 0 & 0 & 1 & 0 & 1 & 2 \\
Some College & 2 & 0 & 3 & 1 & 0 & 6 \\
Associate's & 4 & 0 & 1 & 1 & 0 & 6 \\
Bachelor's & 5 & 2 & 8 & 0 & 0 & 15 \\
Master's & 4 & 0 & 1 & 1 & 0 & 6 \\
Total & 16 & 4 & 16 & 3 & 1 & 40 \\
\hline
\end{tabular}

Chi-Squared Tests

\begin{tabular}{llll}
\hline & Value & df & $\mathrm{p}<0.05$ \\
\hline $\mathrm{X}^{2}$ & 35.50 & 20 & 0.018
\end{tabular}

$\mathrm{N} \quad 40$

Statistically significant association between level of education of participants and sun protection usage.

Finally, contingency tables and Chi-square tests were conducted to test the association between sun protection usage and the 5 CPD-Reaction constructs: intention, social influence, beliefs about capabilities, moral norm, and beliefs about consequences. First, there were no significant associations found between sun protection usage and the CPD-Reaction construct of intention $\left(\mathrm{x}^{2}=19.16, d f 20\right.$, and $\left.\mathrm{p}=0.511\right)$. Table 8 further illustrates these findings. 
Table 8

Contingency Table Testing Association Between Sun Protection Usage and Intention

Intention

Protection

$\begin{array}{lllllll}1 & 3 & 4 & 5 & 6 & 7 & \text { Total }\end{array}$

sunscreen

protective clothing

both

none

$\mathrm{n} / \mathrm{a}$

Total

$\begin{array}{llll}1 & 0 & 7\end{array}$

$\begin{array}{lllllll}0 & 0 & 0 & 2 & 1 & 1 & 4\end{array}$

$\begin{array}{lllllll}0 & 1 & 8 & 0 & 4 & 3 & 16\end{array}$

$\begin{array}{lllllll}0 & 0 & 2 & 0 & 1 & 0 & 3\end{array}$

$\begin{array}{lllllll}0 & 0 & 0 & 0 & 0 & 1 & 1\end{array}$

Chi-Squared Tests

\begin{tabular}{llll}
\hline & Value & df & $\mathrm{p}<0.05$ \\
\hline $\mathrm{X}^{2}$ & 19.16 & 20 & 0.511
\end{tabular}

$\mathrm{N}$

40

No significant associations found between sun protection usage and intention

Second, there were no significant associations found between sun protection usage and the CPD-Reaction construct of beliefs about consequences $\left(\mathrm{x}^{2}=10.44, d f 12\right.$, and $\left.\mathrm{p}=0.577\right)$. Table 9 further illustrates these findings. 
Table 9

Contingency Table Testing Association Between Sun Protection Usage and Beliefs About Consequences

\begin{tabular}{|c|c|c|c|c|c|c|c|c|}
\hline \multirow{2}{*}{\multicolumn{2}{|c|}{ Protection }} & & & \multicolumn{4}{|c|}{$\begin{array}{l}\text { Beliefs about } \\
\text { consequences }\end{array}$} & \multirow[b]{2}{*}{ Total } \\
\hline & & & & $\overline{4}$ & 5 & 6 & 7 & \\
\hline \multicolumn{4}{|c|}{ sunscreen } & 1 & 1 & 1 & 13 & 16 \\
\hline \multicolumn{4}{|c|}{ protective clothing } & 1 & 0 & 1 & 2 & 4 \\
\hline \multicolumn{4}{|c|}{ both } & 0 & 0 & 3 & 13 & 16 \\
\hline \multicolumn{4}{|c|}{ none } & 1 & 0 & 1 & 1 & 3 \\
\hline \multicolumn{4}{|l|}{$\mathrm{n} / \mathrm{a}$} & 0 & 0 & 0 & 1 & 1 \\
\hline \multicolumn{4}{|c|}{ Total } & 3 & 1 & 6 & 30 & 40 \\
\hline \multicolumn{9}{|c|}{ Chi-Squared Tests } \\
\hline & Value & $\mathrm{df}$ & $\mathrm{p}<0.05$ & & & & & \\
\hline$\overline{X^{2}}$ & 10.44 & 12 & 0.577 & & & & & \\
\hline $\mathrm{N}$ & 40 & & & & & & & \\
\hline
\end{tabular}

Third, there were no significant associations found between sun protection usage and the CPD-Reaction construct of social influence $\left(\mathrm{x}^{2}=17.92, d f 16\right.$, and $\left.\mathrm{p}=0.329\right)$. Table 10 further illustrates these findings. 
Table 10

Contingency Table Testing Association Between Sun Protection Usage and Social Influence

\begin{tabular}{lllllll}
\hline \multirow{2}{*}{ Protection } & \multicolumn{7}{l}{ Social influence } & & \\
\cline { 2 - 6 } sunscreen & 3 & 4 & 5 & 6 & 7 & Total \\
protective clothing & 2 & 12 & 1 & 0 & 1 & 16 \\
both & 0 & 3 & 0 & 1 & 0 & 4 \\
none & 5 & 6 & 4 & 1 & 0 & 16 \\
n/a & 0 & 2 & 1 & 0 & 0 & 3 \\
Total & 1 & 0 & 0 & 0 & 0 & 1 \\
\hline
\end{tabular}

Chi-Squared Tests

\begin{tabular}{llll}
\hline & Value & df & $\mathrm{p}<0.05$ \\
\hline $\mathrm{X}^{2}$ & 17.92 & 16 & 0.329
\end{tabular}

$\mathrm{N} \quad 40$

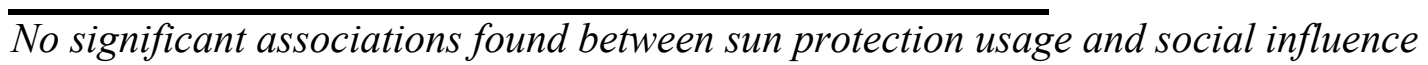

Also, there were no significant associations found between sun protection usage and the CPD-Reaction construct of beliefs about capabilities $\left(\mathrm{x}^{2}=14.91, d f 16\right.$, and $\left.\mathrm{p}=0.531\right)$. Table 11 further illustrates these findings. 
Table 11

Contingency Table Testing Association Between Sun Protection Usage and Beliefs About Capabilities

\begin{tabular}{lllllll}
\hline & \multicolumn{5}{c}{ Beliefs about capabilities } & \\
\cline { 2 - 6 } Protection & 3 & 4 & 5 & 6 & 7 & Total \\
\hline sunscreen & 2 & 6 & 5 & 1 & 2 & 16 \\
protective clothing & 0 & 1 & 2 & 0 & 1 & 4 \\
both & 1 & 5 & 7 & 3 & 0 & 16 \\
none & 0 & 1 & 1 & 0 & 1 & 3 \\
n/a & 0 & 0 & 0 & 1 & 0 & 1 \\
Total & 3 & 13 & 15 & 5 & 4 & 40 \\
\hline
\end{tabular}

Chi-Squared Tests

\begin{tabular}{llll}
\hline & Value & df & $\mathrm{p}<0.05$ \\
\hline $\mathrm{X}^{2}$ & 14.91 & 16 & 0.531
\end{tabular}

$\mathrm{N} \quad 40$

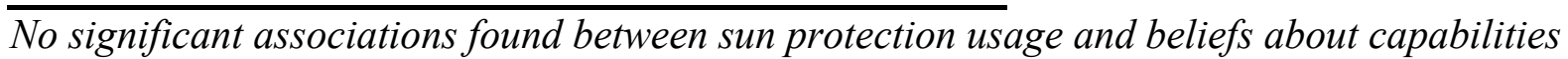

Finally, there were no significant associations found between sun protection usage and the CPD-Reaction construct of moral norm $\left(\mathrm{x}^{2}=18.79, d f 20\right.$, and $\left.\mathrm{p}=0.536\right)$. Table 12 further illustrates these findings. 
Table 12

Contingency Table Testing Association Between Sun Protection Usage and Moral Norm

\begin{tabular}{|c|c|c|c|c|c|c|c|}
\hline \multirow[b]{2}{*}{ Protection } & \multicolumn{6}{|c|}{ Moral Norms } & \multirow[b]{2}{*}{ Total } \\
\hline & 1 & 3 & 4 & 5 & 6 & 7 & \\
\hline sunscreen & 1 & 6 & 5 & 1 & 0 & 3 & 16 \\
\hline protective clothing & 0 & 0 & 2 & 0 & 1 & 1 & 4 \\
\hline both & 0 & 0 & 10 & 1 & 2 & 3 & 16 \\
\hline none & 0 & 1 & 1 & 0 & 0 & 1 & 3 \\
\hline $\mathrm{n} / \mathrm{a}$ & 0 & 0 & 0 & 0 & 0 & 1 & 1 \\
\hline Total & 1 & 7 & 18 & 2 & 3 & 9 & 40 \\
\hline
\end{tabular}

Chi-Squared Tests

\begin{tabular}{cccc}
\hline & Value & df & $\mathrm{p}<0.05$ \\
\hline $\mathrm{X}^{2}$ & 18.79 & 20 & 0.536 \\
$\mathrm{~N}$ & 40 & &
\end{tabular}

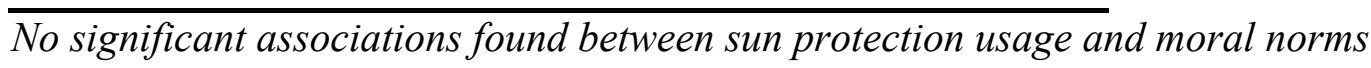

\section{Objective 5}

The final objective was to give the participants a questionnaire, Personal Practice Change (PPC) survey, focusing on the likelihood and feasibility of implementing the education derived from the intervention into daily personal practice within the organization. These questions were written in a Yes/No format, and can be found in Appendix G. When reviewing the results of the PPC, approximately $35 \%$ of the participants answered yes to question 1, which focused on his or her likelihood to use sun prevention/sunscreen during outdoor time at the facility. The majority of the participants answered yes to questions 2 and 3, respectively (100\% and 98\%). Question 2 focused on the participants' likelihood to encourage the children to use sun prevention/sunscreen during their time spent outdoors, and question 3 centered on the participants' feeling regarding 
the organizational support for the practice change of using sun prevention/sunscreen. These results are illustrated in Figure 9.

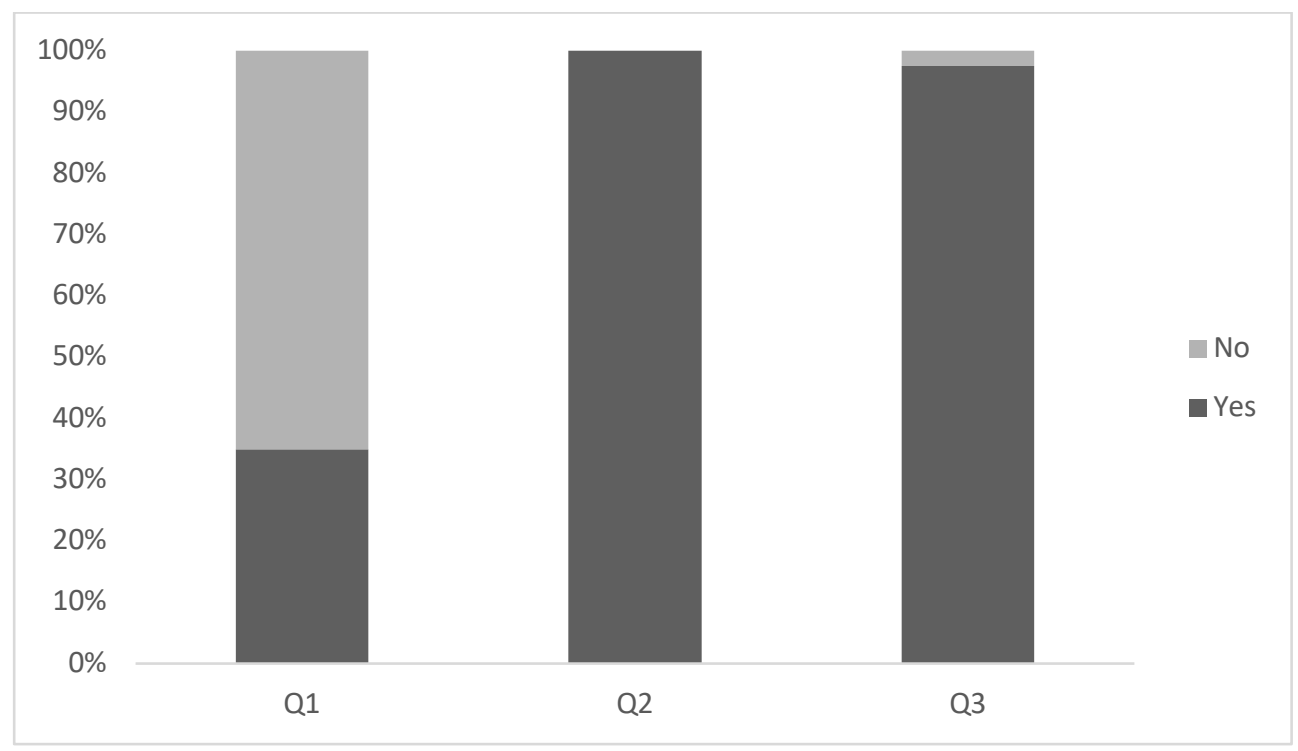

Figure 9. Personal Practice Change survey results

\section{Results and Discussion}

Analysis of the pretest-posttest results indicate statistically significant increase in test scores after the Sun Smart U skin cancer prevention educational intervention performed with the participants at the organization (CI 95\%,d=0.463, $\mathrm{p}=0.006$ ). Thus, a predetermined curriculum focusing on sun prevention education for adult health care providers can significantly increase knowledge of sun protection factors when implemented in a similar setting. These results support the clinical significance of targeting health care professionals that deliver direct care to their patients and could potentially impact the population of the patients they serve. A key facilitator that made this goal achievable was the convenience sample $(\mathrm{N}=40)$ of participants employed by the facility. Because of current circumstances, these individuals were readily available to receive the educational intervention and had some experience with the targeted child population. A key barrier identified in this analysis was the ability to retain all 40 participants throughout the entire educational intervention. Because of the nature of the facility, overhead pages and work 
demands would occasionally cause some participants to step out and re-enter after missing some content covered by the teaching. This has been identified by the author as an extraneous variable potentially affecting the results of the pretest-posttest scores.

Results of measurable objective 3 focused on the participant's intent to change behaviors incorporating sun prevention into daily professional practice post educational intervention. Each of the 5 constructs from the CPD-Reaction questionnaire were measured to determine which one(s) would have the highest impact on the participants' ability to change his or her individual behavior. Of these 5 social constructs, Beliefs About Consequences appeared to be the most prominent $($ mean $=6.4, \mathrm{SED}=0.21$, and $\mathrm{SD}=1.3$ ) and most likely to affect sun protective behavior change with the population under study. These results indicate that if the participants' behavior were to be measured again in the future, his or her beliefs about the consequences of not participating in sun prevention behaviors would guide their personal intent to change these behaviors. Ideally, after the Sun Smart U curriculum was delivered, the participants would begin considering the factors associated with lack of sun protection and skin cancer prevention (e.g. lack of sunscreen usage, no protective clothing, indoor/outdoor tanning). Realizing the consequences and severity of his or her lack of action against sun protection, the individual would change to positive behaviors avoiding direct sun exposure; thus, decreasing the chances of developing skin cancer. Ultimately, the beliefs about consequences would naturally impact the health care professional to practice beneficence for their patient populations (e.g. children) by educating and encouraging positive sun prevention behaviors. A key facilitator driving this result could be the fear of not engaging in sun prevention activities that would negatively impact health. On the contrary, a barrier may be the belief that the consequences do not outweigh the actions or choices of the individual engaging in poor sun exposure behaviors. 
Examining the potential associations between the demographic factors of age, gender, and education with sun protection usage revealed that participants with a higher level of education were significantly more likely to use sunscreen, protective clothing, or both as compared to those individuals without higher education $\left(\mathrm{x}^{2}=35.50, d f=20, \mathrm{p}=0.018\right)$. Thus, it seems justified that those individuals attaining higher education would engage in better decisionmaking and positive behavior change to avoid skin cancer and the negative effects of sun overexposure. In addition, a positive assumption from this data could be that health care professionals with higher education would be more likely to role model positive sun prevention behaviors to their patients.

Analyzing the results of the Personal Practice Change survey, data revealed that $100 \%$ of the participants would encourage children within the current organization to use sun prevention and sunscreen outdoors. Ninety-eight percent of the participants felt that the organization would support a practice change of using sun prevention and sunscreen measures on site. For an organization of this size and nature, this speaks volumes regarding the positive organizational culture, and appears to be synonymous with the mission and vision set forth by the organization. On the contrary, only $35 \%$ of participants indicated likeliness to use sun prevention and/or sunscreen during their time outdoors at the facility. Assumptions could be made that although the participants view the organization would positively support or implement sun prevention efforts, his or her personal participation would not be likely. However, it was unanimous that all participants would encourage the children to engage in such behaviors. This information could be conflicting, and thus hinder role modeling from the health care professionals providing care in the facility. This lack of personal participation in sunscreen protection is perceived as a barrier to sun prevention efforts within the organization, and would need to be explored further for strategies to support employee buy-in. 
Conclusions from this analysis show that a preset curriculum focusing on sun prevention education can increase a health care professional's knowledge regarding positive sun protection behaviors. With this sample population, there was a significant $(\mathrm{p}=0.018)$ association between sun protection usage and education level indicating that individuals with an Associate's, Bachelor's, and/or Master's Degree engage in more sun protective measures compared to those individuals without higher levels of education. Although the CPD-Reaction tool has been proven to be a valid and reliable tool for measuring the level of the health care professional's intent of translating the proposed behavioral changes into daily practice (Appendix F), there were no significant associations found between its 5 social constructs and sun protection usage in this study. Finally, the evidence from the Personal Practice Change survey indicates that the although the employees of the facility support the positive organizational culture, they themselves would not be likely to personally engage in sun protection behaviors at the facility. However, of positive note, the employees would fully support educating and encouraging the children at the organization to partake in sun protection activities.

The purpose of this project was to implement an educational intervention using a predetermined curriculum from The Skin Cancer Foundation to increase hospital staff knowledge regarding sun preventive measures. This project has been implemented through collaboration with all facility administration (including the director of education) to coordinate this curricular intervention. All participants in the study have been educated regarding the application of sunscreen, as well as other sun protective measures. The measurable goals and objectives of the project regarding communication of sun protection and health promotion to all stakeholders have been met through these educational sessions, as well as sharing the results of the Personal Practice Change (PPC) survey for further consideration by executive management. The staff support has been put into place to ensure intervention sustainability post implementation as 
evidenced by the full Sun Smart U curriculum provided to the facility for further utilization with staff and/or its targeted child and adolescent populations. Therefore, the purpose of this project as indicated in the initial problem statement has been fulfilled, and the evidence presented supports a practice change within the facility with regard to (1) increasing sun protection and skin cancer protection knowledge among staff, and (2) the participants' unanimous indication to encourage the children to use sun prevention/sunscreen during his or her time outdoors.

\section{Future Recommendations for Practice}

Based on the results of this study, recommendations for the participating organization include adopting the Sun Smart U curriculum on sun protection and skin cancer prevention into new employee orientation sessions. This would assist the facility with immersing new hires into these preventive practices, which supports the values of the organization as stated in the strategic plan of providing quality patient care, patient-centered atmosphere, ethical treatment of all, innovation, total well-being, and investing in their people. Because the employee participants indicated they would be $100 \%$ supportive of encouraging the children to use sun prevention/sunscreen during outdoor activities, the ideas this facility could expand upon would be twofold: (1) to provide the Sun Smart U curricular education directly to the children in a classroom setting while residing in the facility; and, (2) placing sunscreen on formulary with the pharmacy at the institution for use on an outdoor sunsafe protocol. These actions would directly be supported by the results indicated by this study and could be considered for further development.

Another avenue to explore for this organization would be to install sunscreen dispensers around the outdoor campus, especially areas directly associated with play activities. Because of the nature of the facility, cameras are currently in place to monitor activity around the facility; hence, the children and adolescent patients could be monitored for his or her actual sunscreen 
usage after Sun Smart U curricular education delivery. These actions would support the organizational culture of safety and prevention, as well as promote positive health care behaviors in employees and the patient population. Furthermore, this would be a pioneered action on behalf of the facility in an attempt to educate and influence children and health care providers through sun preventive measures, which in turn parallels the organization's strategic plan of innovation.

\section{Evaluation of Theoretical Framework Integration}

Kotter's model (1996) of leading change was utilized throughout the implementation of this project. The first step in the model included establishing a sense of urgency among the participants in the study regarding the need for sun prevention education and personal practice behavior change within the facility. This was successful with regard to the level participation $(\mathrm{N}=40)$ for the intervention, as well as the support from the director of education and supervisory staff for assisting with scheduling for the educational sessions. Secondly, there were team members put into place with enough power to effectively lead the behavior change efforts, as indicated in Kotter's (1996) second step. Steps 3 and 4 focused on developing and communicating a vision and strategy to help direct the change effort (Kotter, 1996). The vision of improving the health behaviors of the employees and their patients was communicated via the Sun Smart U educational intervention. The importance of sun protection and skin cancer prevention was the dominating message of the sessions.

Post educational intervention is where Kotter's (1996) theory would indicate elimination of obstacles preventing the change from taking place. Those participants involved in the educational intervention will now be the champions for moving the vision of change forward within the facility. A follow-through is needed here with recognition of short-term wins recognizing those individuals both role-modeling and promoting sunsafe behavior to the patient 
population. The final step in Kotter's model (1996) involves anchoring the new behavior in the culture of the organization. This step will begin to unfold as the Sun Smart U curriculum is integrated into monthly new employee orientations, and all health care providers and staff understand this as a competency for practice within the organization.

\section{Limitations}

During the process of completing the intervention, there were notable limitations identified. For example, the project itself was designed to be implemented in 2 sessions of 4050-minute intervals. This would have completed the needed sample size for the project. However, because of time constraints, holiday schedules, and staffing fluctuations during the time of the planned intervention, adjustments had to be made allowing for 4 sessions instead of the previously planned 2 sessions. This still resulted in the same sample size $(\mathrm{N}=40)$ needed for data analysis; although, this could have had an impact on participants' answers. An unintended consequence may have been alterations in content delivery due to the number of times the presentation was given. Although the same person performed the instructional intervention, subsequent lectures may have been subconsciously adapted due to comfort level, consistency, and repetition. Also, participant \#026 was eliminated from data analysis on the Sun Smart U pretest-posttest True/False paired t-test due to incompletion. However, this was reported in the results section of the data and was accounted for during the analytical process. Of notable mention, the participants in this intervention were employed at a medical facility and provided both direct and indirect care for the patient population, therefore the $\sim 93 \%$ pretest score could have been a ceiling effect. On a positive note, the high scores earned by the adult participants could indicate an understanding of sun protection and thus the need to use sunscreen. It would be disheartening if health care providers scored low (especially those directly involved in patient care) while working with the target population of children. Although the results of this study 
were statistically significant, this does not indicate clinical significance. Again, results from this study show no significant associations between sun protection usage and the 5 constructs of the CPD-R tool. It is a future recommendation from this study that larger sample sizes be used to support the validity, reliability, and dissemination results; hence, a follow-up CPR-Reaction questionnaire would be helpful to assess the actual intended sun preventive behavior change as recommended by Légaré et al., 2017).

\section{Implications for Future Practice}

The DNP student initiating the change project within the participating facility is the first phase of sun prevention awareness with health care professionals in the health care organization. Steps need to be taken to ensure the Sun Smart U curriculum remains in place. First, the director of education has been given a 3-ring binder containing the Skin Cancer Foundation Sun Smart U curriculum (2017) for both implementation in new employee orientation as well as in the classroom with residential children. Next steps would involve placing sunscreen on formulary by meeting with the director of pharmacy services and working with policy and procedure committees in developing an outdoor play protocol for the children. Finally, sun screen dispensers would need to be placed outdoors around the facility, and their usage monitored for actual participation and behavior changes of both employees and patients. This can be facilitated by individual incentives placed throughout the facility, such as monthly employee recognition programs or other appropriate rewards for the children (e.g. parties, field trips, etc.). All of these actions are feasible and could be reinforced via yearly competencies for the employees. Future stakeholders ensuring the success and sustainability for this project include the Chief Executive Officer, Executive Vice Presidents, Director of Pharmacy, Director of Education, employees, and patients of the participating facility. Altogether, the elements of the sustainability to increase sun 
safe knowledge and intent to change sun protective behavior are supported by the mission, vision, and strategic plan of the organization.

In examining possible application of this project in other settings, the level of education and ability to follow-up on intent to change behavior should be considered. For instance, those persons without a health care background or lower levels of education should be targeted. This may assist tin increasing the impact of preventive care with lower education groups through reaching adults and children less likely to have formal education. An ultimate goal of this project, going forward in other settings, would be to implement the Sun Smart U curriculum teaching in the public-school system. This would bring education to the classroom while impacting teachers and parents with the knowledge to partake in sun protection and skin cancer prevention methods. Legislation would need to be considered allowing sunscreen dispensers to be placed in the school systems. Additionally, this would involve educating both local and state representatives on the importance of early detection and prevention of skin cancer.

\section{Attainment of DNP Essentials}

This educational intervention for skin cancer prevention with hospital staff has been evaluated according the attainment of the Doctor of Nursing Practice Essentials (AACN, 2006). The Essentials (I-VIII) outline clear curricular elements and competencies in which the DNP student must meet in order to both practice within the discipline of nursing and do so within the depth and focus of the particular role in which the student is preparing. Elements depicting this particular capstone project's successful connection with the DNP Essentials as related to administrative leadership are outlined in the following summaries below.

\section{Essential I. Scientific Underpinnings for Practice}

Essential I focuses on connecting nursing with the principles that govern human behavior, life-processes, well-being, and interaction with his or her environment (AACN, 2006). 
This project focused on sun protective behaviors as an identified health prevention method to prevent skin cancer. By implementing an educational intervention such as the Sun Smart U curriculum, the author attempted to increase knowledge about a specific disease process and facilitate positive behavior change through use of the Continued Professional DevelopmentReaction (CPD-R) tool measuring human intent to change behavior. This was performed using John P. Kotter's Theory of Leading Organizational Change (1996) and was grounded in foundational nursing practice.

\section{Essential II. Organizational and Systems Leadership for Quality Improvement and}

\section{Systems Thinking}

Essential II focuses on the development of care delivery approaches that meet both current and future needs of the targeted patient populations within an organizational context while executing cost effective strategies (AACN, 1996). An organizational assessment was performed with budgetary and fiscal considerations to measure the feasibility of implementing project change focusing on sun protective behaviors. The employees' perceptions of facilitating the change with the child population were high (100\%); however, the likelihood of the employees themselves participating in change themselves were low (35\%). Almost all participants $(\mathrm{N}=40)$ indicated that the organization would support the behavior change $(98 \%)$. The cost was very low, with exception to lunches provided for the participants during the actual educational interventions. Because the Sun Smart U Curriculum is free and easily accessible to the facility, the change would appear to be fiscally responsible and congruent the organization's mission, vision, and strategic plan.

\section{Essential III. Clinical Scholarship and Analytical Methods for Evidence-Based Practice}

Essential III focuses on clinical discovery that makes connections across disciplines through the scholarship of integration, with the application and dissemination of gleaned 
knowledge to nursing practice (AACN, 2006). Through the completion of this capstone project, new findings have indicated that an educational intervention focusing on skin cancer prevention improves knowledge of health care professional regarding sun protective behavior. In addition, the participant's plan engage in sun protective behavior was statistically significant $(\mathrm{p}=0.018)$ as related to their education level. This discovery led to the conclusion that the same intervention should be performed with individuals of a lower educational level for more impact. Analytical methods included preforming a literature review to support the need for sun protective education with this target population. The data were analyzed to examine intent to change behavior, as well as knowledge level both pre and post intervention. Findings contribute to the body of evidence-based practice knowledge as it relates to the context of nursing.

\section{Essential IV. Information Systems/Technology and Patient Care Technology for the Improvement and Transformation of Health Care}

Essential IV focuses on the ability to improve patient care and healthcare systems through the use of information systems and/or technology (AACN, 2006). In regard to this essential, this project was evaluated on the future implications for practice to create an outdoor play protocol for the children using sunscreen on pharmacy formulary. This would involve collaboration with both nursing, pharmacy, and information technology services to develop an electronic protocol for use. Also, the data analysis of the current project included an adequate understanding of JASP (Version 0.9.2, 2018) which is an open-source graphical program designed for familiar users of SPSS. This data analytic tool can be used in the author's future practice for additional projects. Of notable mention, completion of this project required consulting regularly with a statistician for precise interpretation of results

\section{Essential V. Health Care Policy for Advocacy in Health Care}


Essential $\mathrm{V}$ focuses on advocating for health care policy to address and improve standards of care, organizational policy, and care delivery outcomes (AACN, 2006). Future implications for this project discussed assessment and creation of an outdoor play protocol for the children at the facility. This project could disseminate findings to the public-school system implementing the Sun Smart U curriculum. Findings from this pilot project indicate that a larger study sample with perhaps lower education levels could further benefit from the sun protection educational intervention. Results also support policies at the both the national and state levels. For example, tanning bed legislation banning children under the age of 18 years was recently passed in the state of West Virginia. This preliminary law is the first in West Virginia supporting sun prevention measures.

\section{Essential VI. Interprofessional Collaboration for Improving Patient and Population Health Outcomes}

Essential VI focuses on collaborating effectively within interprofessional teams to create change in complex delivery systems (AACN, 2006). Throughout the implementation of this project, it proved necessary to communicate with the CEO of the organization, director of education, nursing supervisor, Capstone Chairperson and committee, IRB members, statistician, and employees and staff of the participating organization. Without the interdisciplinary communication and cooperation of all participants contributing to the outcomes of this project, the discoverable outcomes leading to evidence-based practice change may not have been possible.

\section{Essential VII. Clinical Prevention and Population Health for Improving the Nation's} Health

Essential VII focuses on the health promotion and/or risk reduction of persons in the context of his or her environmental, cultural, or socioeconomic dimension of health (AACN, 
2006). This project examined the significance of demographic data against the participant's knowledge level of sun protective behaviors as well as his or her intent to change behavior. Results indicated that those with a higher level of education were significantly $(p=0.018)$ more likely to engage in sun protective behaviors. Future implications identified focusing on those with lower education and socioeconomic status to improve his or her knowledge level of sun protective behavior and skin cancer prevention.

\section{Essential VIII. Advanced Nursing Practice}

Essential VIII focuses on comprehensively integrating the complex knowledge of healthcare within the context of advanced nursing practice (AACN, 2006). This project involved developing a relationship with administrative leadership at the participating facility and assessing the need of the current population with regards to sun protection education and skin cancer prevention. An educational intervention for skin cancer prevention with hospital staff was designed and implemented using pretest-posttest methodology, intent to change behaviors (CPD-R), and Personal Practice Change (PPC) assessment. Advanced levels of clinical judgement were applied to data collection during the statistical analysis phase of the project. Results of the findings suggest that although there was an increase in sun protection knowledge, those with a higher level of education were significantly more likely to engage in sun protective behaviors. The intent to change behavior could be re-evaluated in 3 months, as recommended by Légaré et al. (2017) in his development of the CPD-R tool to attain valuable results that would contribute to the purpose of this project.

\section{Summary}

In summary, a predetermined educational intervention focusing on sun protection and skin cancer prevention revealed a statistically significant increase in test scores for pretest to 
posttest knowledge levels (95\% CI, Cohen's $d=0.463, \mathrm{p}=0.006)$. Also, participants with a higher level of education were significantly more likely to engage in sun protective behaviors $\left(\mathrm{x}^{2}\right.$ $=35.50, d f=20, p=0.018)$. Staff were in unanimous agreement that they would provide sun protection to the children in the organization's care. This study addressed two important necessities in skin cancer prevention: the need for a staff intervention to improve knowledge regarding sun safe behaviors, and the future development of an outdoor activities protocol incorporating sunscreen. Results of this study utilizing the Sun Smart U curriculum could be disseminated to other institutions throughout the state of West Virginia in an effort to decrease skin cancer mortality.

Sun preventive behaviors are exponentially essential in the development of children. Instilling early harm reduction behaviors can provide a healthy alternative to life choices these children are exposed to during critical years of maturation. The Skin Cancer Foundation recognizes that early prevention and sun screening behaviors are imperative and should be introduced in the early stages of development (2017). Literature has shown that children's health and development are connected to a social gradient: the better the socioeconomic status, the better the projected outcome (Moore, McDonald, Carlon, \& O'Rourke, 2015). The amount of exposure that a child receives from his or her environment, whether positive or negative, can compromise or promote healthy development. The participating facility recognized the need for children with behavioral health issues to have attention regarding the development of health promotion behaviors. The project reflected this innovation to bring health promotion through sun prevention education to the staff of children in a supervised, residential environment. Therefore, the project presented as a sustainable alternative providing both staff and children with initial healthy habits that can be continued throughout adulthood. 


\section{References}

Acharyya, S., \& Leff, S. (Promoting health of looked after children. British Medical Journal, 320, (7236), 661-662. Retrieved from https://www.ncbi.nlm.nih.gov/pmc/articles/PMC1117693/pdf/661.pdf

Ali, Z., Yousaf, N., \& Larkin, J. (2013). Melanoma epidemiology, biology and prognosis. European Journal of Cancer Supplements, 11 (2), 81-91. Retrieved from http:/www.ncbi.nlm.nih.gov/pmc/articles/PMC4041476/

American Association of Colleges of Nursing (AACN). (2006). The essential of doctoral education for advanced nursing practice. Retrieved from www.aacn.nche.edu

American Cancer Society. (2017). Cancer facts and figures 2017. Retrieved from http://www.cancer.org/acs/groups/content/@editorial/documents/document/acspc048738.pdf

American Cancer Society. (2018). Skin cancer prevention and early detection. Retrieved from https://www.cancer.org/cancer/skin-cancer/prevention-and-early-detection.html

American Society for Dermatologic Surgery Association (ASDSA). (2013). Sunscreen use in schools position statement. Retrieved from http://asdsa.asds.net/position$\underline{\text { statement/sunscreen-use-in-school.aspx }}$

Anderson, A.M., Matsumoto, M., Saul, M.I., Secrest, A.M. \& Ferris, L.K. (2018). Accuracy of skin cancer diagnosis by physician assistants compared with dermatologists in a large health care system. JAMA Dermatology, 154 (5), 569-573.

Doi: 10.1001/jamadermatol.2018.0212

Centers for Disease Control and Prevention. (2011). Guidelines for school programs to prevent skin cancer. Retrieved from https://www.cdc.gov/cancer/skin/what_cdc is doing/guidelines.htm 
Centers for Disease Control and Prevention. (2015). Vital signs: Melanoma incidence and mortality trends and projections - United States, 1982-2030. Retrieved from https://www.cdc.gov/mmwr/preview/mmwrhtml/mm6421a6.htm

Crocker, C.., \& Scott, S. (2006). Improving the mental and emotional well-being of looked after children: Connecting research, policy and practice. Journal of the Royal Society for Public Health, 126, (1), 18-23. Retrieved from http://journals.sagepub.com/doi/pdf/10.1177/1466424006061169

Darling, M, \& Ibbotson, S.H. (2002). Sun awareness and behavior in healthcare professionals and the general public. Clinical and Experimental Dermatology, 27, 442.444. Retrieved from https://onlinelibrary-wiley-com.www.libproxy.wvu.edu/doi/epdf/10.1046/j.1365$\underline{2230.2002 .01110 . x}$

Davis, R., Loescher, L., Rogers, J., Spartonos, D., Snyder, A., Koch, S. . .Harris, R. (2015). Evaluation of project students are sun safe (SASS): A university student-delivered skin cancer prevention program for schools. Journal of Cancer Education, 30, 736-742. Doi: $10.1007 / \mathrm{s} 13187-014-0742-7$

De Troya Martín, M., Padilla-España, L., Férnandez-Morano, T., Delgado-Sánchez, N., Blázquez Sánchez, N., Rivas-Ruiz, F. . . . De Gálvez-Aranda, M.V. (2016). Sun protection habits and attitudes among healthcare personnel in a Mediterranean population. Journal of Cancer Education, 31, 789-795.

Doi: $10.1007 / \mathrm{s} 13187-015-0913-1$

Dietrich, A. J., Olson, A. L., Sox, C. H., Stevens, M., Tosteson, T. D., Ahles, T. . . Sanson-Fisher, R. (1998). A community-based randomized trial encouraging sun protection for children. Journal of the American Academy of Pediatrics, 102 (6), 1-8.

Doi: 10.1542/peds.102.6.e64 
Dietrich, A. J., Olson, A. L., Sox, C.H., Tosteson, T. D., \& Grant-Peterson, J. (2000).

Persistent increase in children's sun protection in a randomized controlled community trial. Preventive Medicine, 31, 569-574. Doi: 10.1006/pmed.2000.0746

Ferrari, A., Bono, A., Baldi, M, Collini, P., Casanova, M., Pennacchioli, E. . . Bartoli, C. (2005). Does melanoma behave differently in younger children than in adults? A retrospective Study of 33 cases of childhood melanoma from a single institution. Pediatrics, 115, (3), 649-654. Doi: 10.1542/peds.2004-0471

Ferris, L.K., Saul, M.I., Lin, Y., Ding, F., Weinstock, M.A., Geller, A.C. ...Kirkwood, M. (2017). A large skin cancer screening quality initiative: Description and first-year outcomes. JAMA Oncology, 3 (8), 1112-1115. Doi: 10.1001/jamaoncol.2016.6779

Ghissassi, F., Baan, R., Straif, K, Grosse, Y., Secretan, B., Bouvard, V. . . Cogliano, V. (2009). A review of human carcinogens - part D: radiation. Lancet Oncology, 10 (8), 751-752. Retrieved from https://doi-org.www.libproxy.wvu.edu/10.1016/S1470-2045(09)70213-X Glanz, K., Steffen, A.D., Schoenfeld, E. \& Tappe, K.A. (2013). Randomized trial of tailored skin cancer prevention for children: The project SCAPE family study. Journal of Health Communication, 18, 1368-1383. Doi: 10.1080/10810730.2013.778361

Godar, D., Urbach, F., Gasparro, F., \& Van der Leun, J. (2003). UV doses of young adults. Photochem Photobiol, 77, (4), 453-457.

Godin, G., Belanger-Gravel, A., Eccles, M., \& Grimshaw, J. (2008). Healthcare professionals’ intentions and behaviours: A systematic review of studies based on social cognitive theories. Implementation Science, 3 (36), 1-12. Doi: 10.1186/1748-5908-3-36

Gritz, E.R., Tripp, M.K., James, A.S., Harrist, R.B., Mueller, N.H., Chamberlain, R.M., \& Parcel, G.S. (2007). Effects of a preschool staff intervention on children's sun protection: Outcomes of sun protection is fun! Health Education \& Behavior, 34 (4), 562-577. 
Doi: $10.1177 / 1090198105277850$

Highland Hospital. (2017). About. Mission. Vision. Values. Retrieved from http://highlandhosp.com/about-highland/

Hoffman, R.G. III, Rodrique, J.R. \& Johnson, J.H. (2000). Effectiveness of a school-based program to enhance knowledge of sun exposure: Attitudes toward sun exposure and sunscreen use among children. Children's Health Care, 28, (1) 69-86. Retrieved from http://web.b.ebscohost.com.www.libproxy.wvu.edu/ehost/detail/detail?vid=2\&sid=aa8db 4dd-d7cc-4241-a97a-467a40c544bd\%40pdc-vsessmgr03\&bdata $=\#$ AN $=107185663 \& d b=c 8 h$

JASP Team. (2018). JASP (Version 0.9.2). [Computer software].

Kotter, John P. (1996). Leading change. Boston, MA: Harvard Business School Press.

Kotter, J. \& Cohen, D. (2002). The heart of change: Real-life stories of how people change their organizations. Boston, MA: Harvard Business School Press.

Lee, T.K., Rivers, J.K., \& Gallagher, R.P. (2005). Site-specific protective effect of broadspectrum sunscreen on nevus development among white schoolchildren in a randomized trial. Journal of the American Academy of Dermatology, 52 (5), 786-792. Doi: $10.1016 /$ j.jaad.2004.12.009

Légaré, F., Borduas, F., Freitas, A., Jacques, A., Godin, G., Luconi, F. . . Grimshaw, J. (2014). Development of a simple 12-item theory-based instrument to assess the impact of continuing professional development on clinical behavioral intentions. Plos One 9:e91013. Retrieved from https://doi.org/10.1371/journal.pone.0091013

Légaré, F., Freitas, A., Turcotte, S., Borduas, F., Jacques, A., Luconi, F. ...Labrecque, M. (2017). Responsiveness of a simple tool for assessing change in behavioral intention after continuing professional development activities. PloS One, 12 (5), e0176678. 
Retrieved from https://doi.org/10.1371/journal.pone.0176678

Levav, L., \& Fitzsimons, G. (2006). When questions change behavior: The role of ease of representation. Psychological Science, 17 (3), 207-213. Retrieved from http://web.b.ebscohost.com.www.libproxy.wvu.edu/ehost/pdfviewer/pdfviewer?vid=3\&si $\underline{\mathrm{d}=60842 \mathrm{aeb}-\mathrm{ed} 2 \mathrm{e}-4 \mathrm{dbb}-\mathrm{aa} 12-460 \mathrm{a} 70 \mathrm{a} 3 \mathrm{dc} 20 \% 40 \text { sessionmgr104 }}$

Maleissye, M. F., Beauchet. A., Saiag, P., Correa, M., Godin-Beeckmann, A., Haeffelin, M, \& Mahe, E. (2013). Sunscreen use and melanocytic nevi in children: A systematic review. Pediatric Dermatology, 30 (1), 51-59.

McComb, S., Jones, C., Smith, A., Collins, W., \& Pope, B. (2016). Designing incentives to change behaviors: Examining college student intent toward healthy diets. Western Journal of Nursing Research, 38 (9), 1094-1113. Doi: 10.1177/0193945916644705

Moore, T.G., McDonald, M., Carlon, L., \& O’Rourke, K. (2015). Early childhood development and the social determinants of health inequities. Health Promotion International, 30, ii102-ii105. Doi: 10.1093/heapro/dav031

Morwitz, V. Johnson, E., \& Schmittlein, D. (1993). Does measuring intent change behavior? Journal of Consumer Research, 20, 46-61. Retrieved from http://web.a.ebscohost.com.www.libproxy.wvu.edu/ehost/pdfviewer/pdfviewer?vid=1\&si $\underline{\mathrm{d}=\mathrm{de} 531 \mathrm{e} 3 \mathrm{~d}-0 \mathrm{eab}-43 \mathrm{e} 6 \text {-afde-7f6c75dbd3ca\%40sessionmgr4009 }}$

Naldi, L., Chatenoud, L., Bertuccio, P., Zinetti, C., Landro, A., Scotti, L., \& Vecchil, C. (2007). Improving sun-protection behavior among children: Results of a cluster-randomized trial in Italian elementary schools. The "Solesi SoleNo-GISED" project. Journal of Investigative Dermatology, 127, 1871-1877. Doi: 10.1038/sj.jid.5700835

Office of Disease Prevention and Health Promotion. (2018). Healthy People 2020: Cancer 
Objectives. Retrieved from https://www.healthypeople.gov/2020/topicsobjectives/topic/cancer/objectives

Osganian, S.K., Parcel, G.S., \& Stone, E.J. (2003). Institutionalization of a school health promotion program: Background and rationale of the CATCH-ON study. Health Education \& Behavior, 30 (4), 410-417. Doi: 10.1177/1090198103252766

Pertl., M., Hevey, D., Thomas, K., Craig, A., Chuinneagain, S., \& Maher, L. (2010). Differential effects of self-efficacy and perceived control on intention to perform skin cancer related health behaviours. Health Education Research, 25 (5), 769-779.

Doi: 10.1093/her/cyq031

Robinson, J.D., Silk, K.J., Parrott, R.L., Steiner, C., Morris S.M., \& Honeycutt C. (2004). Healthcare providers' sun-protection promotion and at-risk clients 'skin-cancerprevention outcomes. Preventive Medicine, 38, 251-257.

Doi: 10.1016/j.ypmed.2003.10.003

Rogers HW, Weinstock MA, Feldman SR, Coldiron BM. (2015). Incidence estimate of nonmelanoma skin cancer (keratinocyte carcinomas) in the US population. JAMA Dermatol, 151(10), 1081-1086.

Stanton, W.R., Saleheen, H.N., O’Riordan, D., \& Roy, C.R. (2003). Environmental conditions and variation in levels of sun exposure among children in child care. International Journal of Behavioral Medicine, 10, (4), 285-289. https://ink-springercom.www.libproxy.wvu.edu/content/pdf/10.1207\%2FS15327558IJBM1004 1.pdf

The Skin Cancer Foundation. (2018). Children and sun protection. Retrieved from https://www.skincancer.org/prevention/sun-protection/children

The Skin Cancer Foundation. (2017). Facts About Sunburn and Skin Cancer. Retrieved from http://www.skincancer.org/prevention/sunburn/facts-about-sunburn-and-skin-cancer 
The Skin Cancer Foundation. (2017). Teacher resources: Sun Smart U. Retrieved from http://www.skincancer.org/prevention/education-program

The Skin Cancer Foundation. (2017). Skin cancer facts \& statistics. Retrieved from http://www.skincancer.org/skin-cancer-information/skin-cancer-facts

US Department of Health and Human Services (USDHHS). (2014). Ultraviolet-radiationrelated exposures: National toxicology program report on carcinogens. Retrieved from

https://ntp.niehs.nih.gov/ntp/roc/content/profiles/ultravioletradiationrelatedexposures.pdf

US Department of Health and Human Services (USDHHS) WVU Cancer Institute. (2016).

West Virginia Cancer Burden Report 2016. Retrieved from https://dhhr.wv.gov/oeps/cancer/Documents/burdenreport2016.pdf

United States Preventive Services Task Force (USPSTF). (2018). Skin cancer: Counseling. Retrieved from https://www.uspreventiveservicestaskforce.org/Page/Document/UpdateSummaryFinal/ski n-cancer-counseling

Wehner, M.R., Chren, M.M., Nameth, D., Choudhry, A., Gaskins, M., Nead, K.T., ...Linos, E. (2014). International prevalence of indoor tanning: A systematic review and metaanalysis. JAMA Dermatology, 150 (4), 390-400. Doi: 10.1001/jamadermatol.2013.6896

West Virginia Legislature. (2017). HB 2520: Prohibiting the use of a tanning device by a person under the age of eighteen. Retrieved from http://www.wvlegislature.gov/Bill_Status/bills_history.cfm?INPUT=2520\&year=2017\&s $\underline{\text { essiontype }=\mathrm{RS}}$

West Virginia Legislature. (2000). Title 64 legislative rules division of health: Series 11 behavioral health centers licensure. Retrieved from 
http://apps.sos.wv.gov/adlaw/csr/ruleview.aspx?document=2677 
Appendix A

Itemized Budget for Proposed Project

\begin{tabular}{|c|c|c|}
\hline Budget Categories & Personal Funds & Organizational Contributions \\
\hline ADMINISTRATIVE COSTS & $\$ 0$ & $\begin{array}{l}\text { Classroom teacher }(2) \$ 25 / \mathrm{hr} .=\$ 50.00 \\
\text { Hospital staff/employees }(40) \text { for } 2 \text { hours of education at } \$ 10 / \mathrm{hr} .=\$ 800.00 \\
\text { Fringe benefits (FICA, Workers Compensation, Health Insurance }=\$ 178.73\end{array}$ \\
\hline \multicolumn{3}{|c|}{$\begin{array}{l}\text { Administrative Justification: Classroom teachers (2) } 1 \text { hour of education for } 2 \text { employees. Also, approximately } 40 \text { hospital staff/healthcare professionals paid for } 2 \text { hours of education each } \\
(2 \text { sessions of } 40-50 \text { minutes). According to the salary provided by the institution, there have been FICA ( } 7.65 \%) \text {, Worker's Compensation ( } 0.54 \%) \text {, and Health Insurance ( } 30.00 \%) \\
\text { calculated into hourly wages, as noted above. }\end{array}$} \\
\hline MARKETING & $\$ 50.00$ & $\$ 0$ \\
\hline \multicolumn{3}{|c|}{$\begin{array}{l}\text { Marketing Justification: Marketing for this project will include printing of the posters which will need to be laminated and placed throughout the facility. Also, printing for the curriculum } \\
\text { to develop the notebook that will be used to train staff and leave with the Director of Education for further classes. }\end{array}$} \\
\hline EDUCATIONAL MATERIALS/ INCENTIVES & $\$ 0$ & $\$ 0$ \\
\hline \multicolumn{3}{|c|}{$\begin{array}{l}\text { Educational Materials/Incentives Justification: Educational handouts for staff teaching will be provided by SunSmart U at no cost from the Skin Cancer Foundation. Paper and notebook } \\
\text { with pre-developed curriculum will be provided in kind. }\end{array}$} \\
\hline HOSPITALITY (food, room rentals, etc.) & $\$ 0$ & $\$ 200.00$ \\
\hline \multicolumn{3}{|c|}{$\begin{array}{l}\text { Hospitality Justification: The facility will provide the classroom space for implementing the staff educational intervention. There will be no need for rental materials. Food will be } \\
\text { provided by the facility in a "lunch and learn" type atmosphere. }\end{array}$} \\
\hline \multicolumn{3}{|c|}{$\begin{array}{l}\text { Project Supplies Justification: The pre and post tests will be provided to the staff on printed paper that will be provided by the DNP student at no cost to the facility. Writing materials, } \\
\text { projector, video monitor, and television will be provided by the facility with no extra cost for the purposes of the project. }\end{array}$} \\
\hline TRAVEL EXPENSES & $\$ 0$ & $\$ 0$ \\
\hline \multicolumn{3}{|c|}{ Travel Expenses Justification: There will be no travel cost for implementation of the proposed project. The implementation will occur on-site at the facility. } \\
\hline OTHER & $\$ 0$ & $\$ 0$ \\
\hline \multicolumn{3}{|l|}{ Other Justification: No other expenses identified. } \\
\hline TOTALS & $\$ 70.00$ & $\$ 1.298 .73$ \\
\hline
\end{tabular}


Appendix B

Letter of Support from Participating Facility

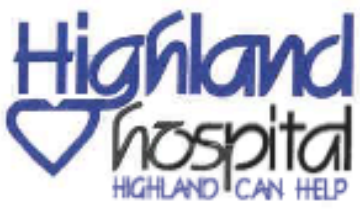

Cynthia A. Persily, PhD

President \& CEO

cpersilyahighlandhosp.com

June 20, 2018

Amy F. Bruce, MSN, RN, NE-BC

Jonas Nurse Leader Scholar

Associate Director of Nursing

Bert Bradford School of Health Sciences

Capito Department of Nursing

2300 MacCorkle Avenue, SE

Charleston, WV 25304

Dear Amy:

It is my pleasure to offer the support and permission of Highland Hospital for you to implement your DNP Capstone project at our facility. The project, entitled An Evidence-Based Educational Intervention for Sunscreen Protection With Hospital Staff: A Pilot Project for System Change, will be implemented with direct care staff in our clinical residential units during the time period of October 2018-December 2018. We understand that this time period is flexible depending on the timing of IRB approval.

During this project, you will work directly with our Director of Training and Organizational Development, Chris Handy RN, who will help to arrange the educational classes with our staff. We will provide you with space to provide the educational intervention, and commit that our staff will be available to you for the class periods.

We look forward to your implementation of this project that will surely result in positive system change for our organization and better outcomes for our patients. Please let me know if there is any additional assistance you require prior to or during implementation.

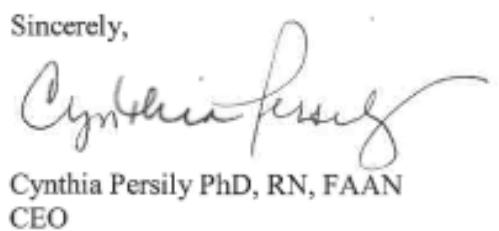

CEO 


\section{Appendix C}

Timeline for Proposed Project

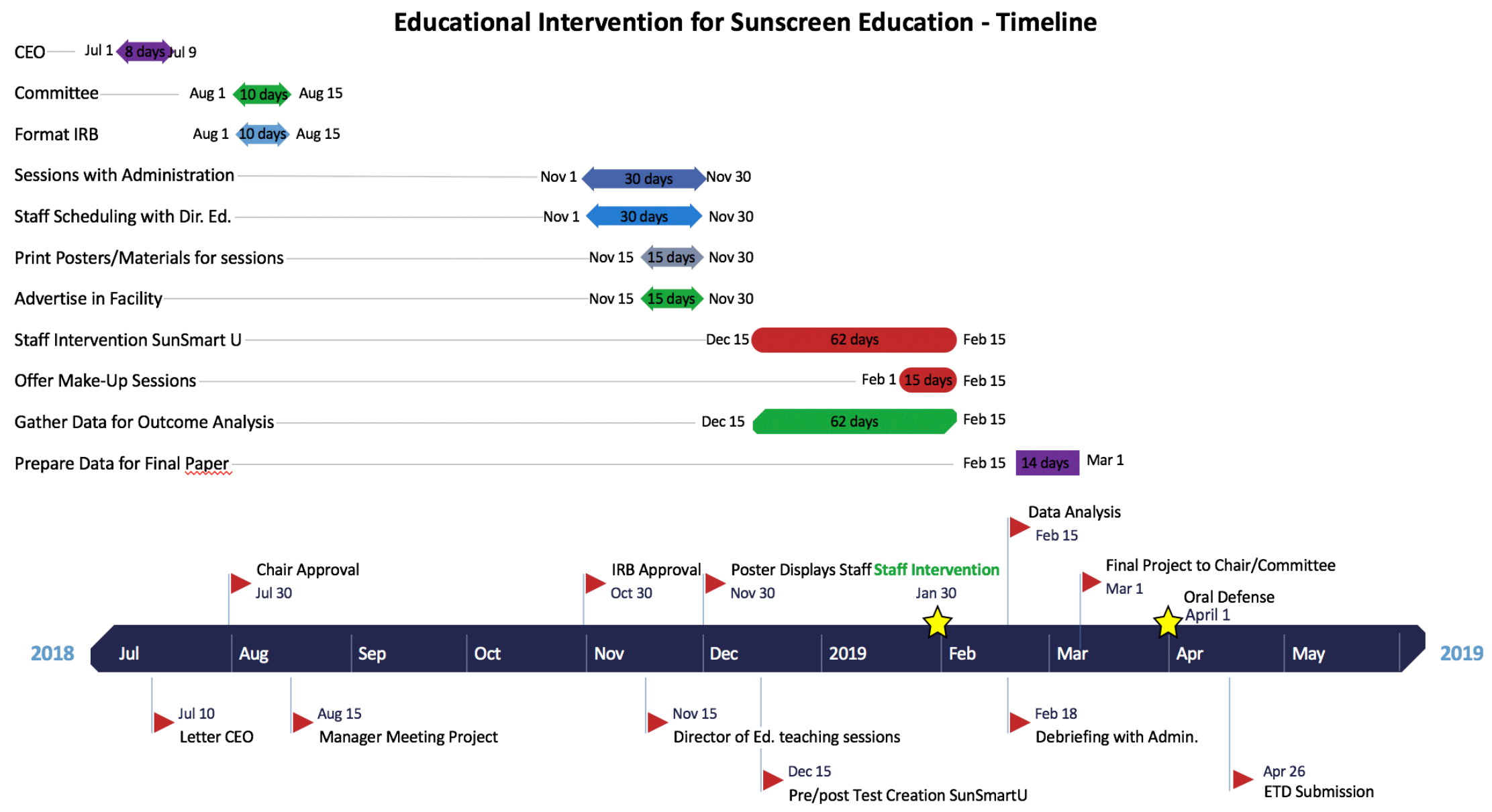




\section{Appendix D}

Sun Smart U Curriculum Pre-Test/Post-Test

Testing Your Knowledge of Skin Cancer

Date:

Code Number:

Please answer the questions by marking the box indicated 'True' or 'False' based on your current knowledge of the subject matter.

1. Skin cancer is rare.

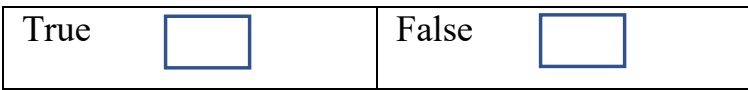

2. You can die from skin cancer.

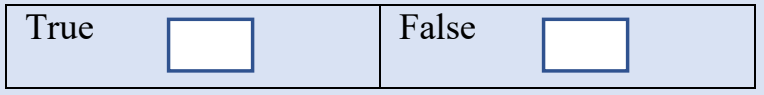

3. Skin cancer is a disease that affects only old people.

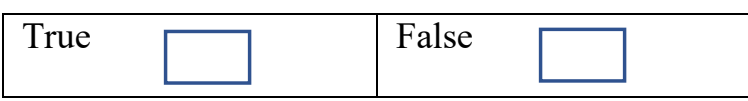

4. People with dark skin can get skin cancer.

5. Experiencing five or more sunburns can double your risk of developing skin cancer.
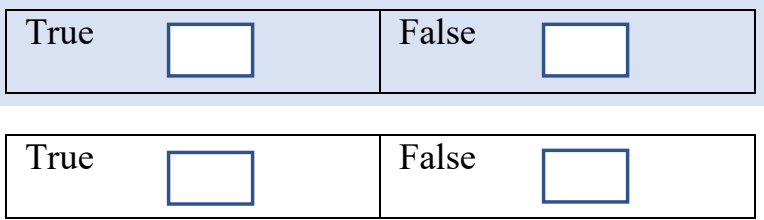

6. Two forms of ultraviolet radiation - UVA and UVB - reach the earth and can cause skin cancer.

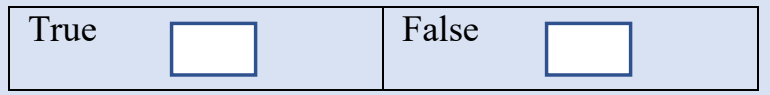

7. Though a sunburn is a sign of sun damage a tan is a sign of health.

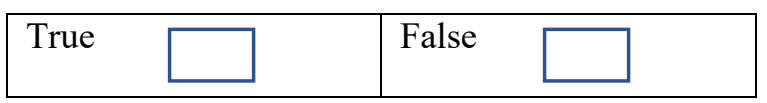

8. Sunshine clears up acne.

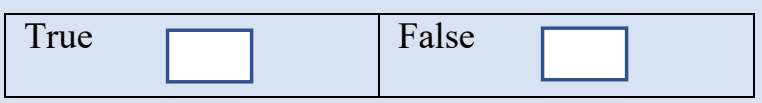

9. "SPF" stands for Sun Protection Factor.

\begin{tabular}{|l|l|}
\hline True & False $\square$ \\
\hline
\end{tabular}

10. Tanning beds are safer for you than sunlight.

\begin{tabular}{|l|l|}
\hline True $\quad \square$ & False $\quad \square$ \\
\hline
\end{tabular}


Appendix E

Email Communication / Permission to Use CPD-R Tool

From: France Légaré < France.Legare@fmed.ulaval.ca>

Sent: Friday, August 17, 2018 12:20 PM

To: Bruce, Amy

Cc: France Légaré; Lysa Bergeron; Hubert Robitaille

Subject: RE: Permission to use CPD-reaction questionnaire

\section{Dear Amy}

Thank you for your email and interest in the instrument

I am happy for you to use this instrument

I will ask Lysa to send you the most updated information we have on the instrument as it has been

translated in 8 languages and is now being presented in publications by diverse groups around the world Here you will find : user manual and papers; Lysa will add links to papers from other groups on this page as well shortly http://www.decision.chaire.fmed.ulaval.ca/en/research/projects/cpd/

Don't hesitate if you need help

Best regards

Second update of our Cochrane review on implementation of shared decision making is out 2018/07/19: https://www.cochrane.org/news/lead-author-interventions-increasinq-use-shared-decision-makinqhealthcare-professionals

France Légaré, B. Sc. Arch, MD, MSc, PhD, CCMF, FCMF Tier 1 Canada Research Chair in Shared Decision Making and Knowledge Translation

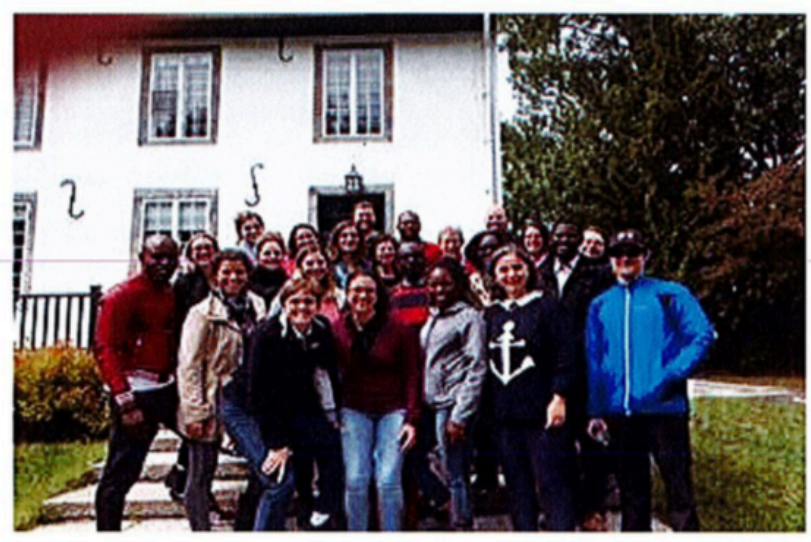

"Deo favente haud pluribus

impar”

:P.:: UNIVERSITE :日. 


\title{
Appendix F
}

Intention to Change Professional Practice Incorporating Sun Protective Behaviors

\author{
Modified CPD-R Questionnaire
}

Date:

Code Number:

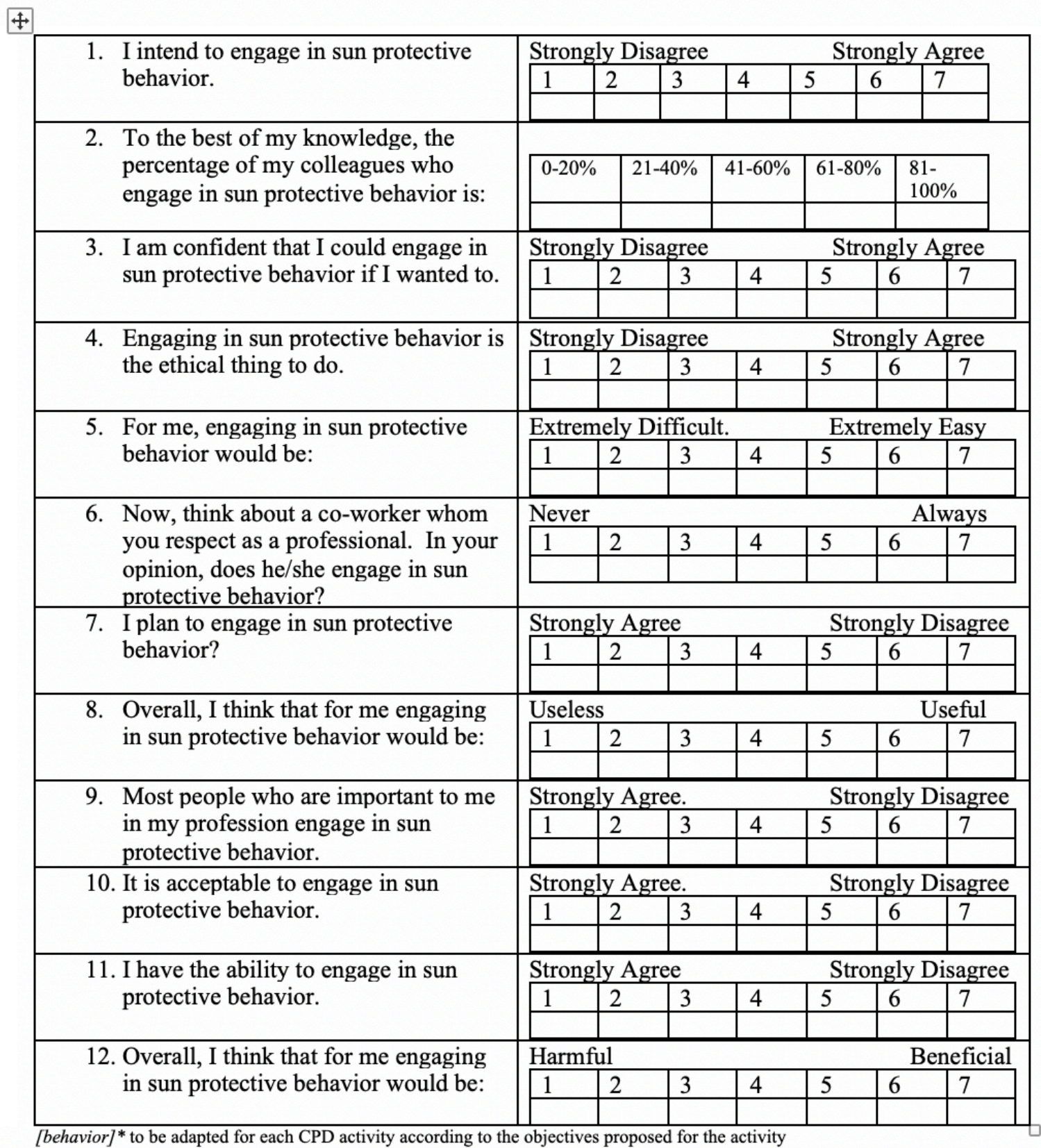

The CPD reaction questionnaire was funded by a Partnership for Health System Improvement grant from the Canadian Institutes of Health Research (CIHR; 2010-2013; grant \# 200911PHE-216868-CFBA-19158) and by the Ministére de la Santé at des Services Sociaux du Québec (MSSS), QC, Canada.

The CPD Reaction Questionnaire ㅇ 2013 Université Laval 
Appendix G

Personal Practice Change Survey

Date:

Code Number:

Please answer the following questions to assist with practice change efforts within your organization:

Q1. Are you likely to use sun prevention/sunscreen during your outdoor time at this facility?

Yes

No

Q2. Will you encourage children to use sun prevention/sunscreen during his/her time outdoors?

Yes

No

Q3. Do you feel the organization will support a practice of using sun prevention/sunscreen?

Yes

No 


\section{Appendix $\mathrm{H}$}

\section{Demographic Data Sheet}

Date:

Code Number:

\section{Directions:}

A quantitative study is being conducted exploring knowledge of sun prevention behaviors, and intent to change behaviors in daily practice. Please answer the following questions by marking all that apply. Enter the date and your code number in the space provided. Please answer every question by circling the answer that best describes you. When indicated select all responses that apply to you and your personal experience.

Q-1. What is your age today?

Q-2. Identify your gender

Q-3. What is the highest level of education you have completed?
A. Some high school
E. College - Associate's Degree
B. High School
F. College - Bachelor's Degree
C. Vocational or technical school
G. Master's Degree
D. Some college
H. Post Master's
E. College -Associates Degree
I. Doctoral Degree
F. College - Bachelor's Degree

Q-4. On a typical day, how many hours to you engage in outdoor activities?

Q-5. On a typical day, how many hours do you spend in direct sun?

Q-6. When in direct sun, do you prefer to wear:__ sunscreen? _ protective clothing?__ both 


\section{Appendix I}

Sun Smart U Curriculum from The Skin Cancer Foundation
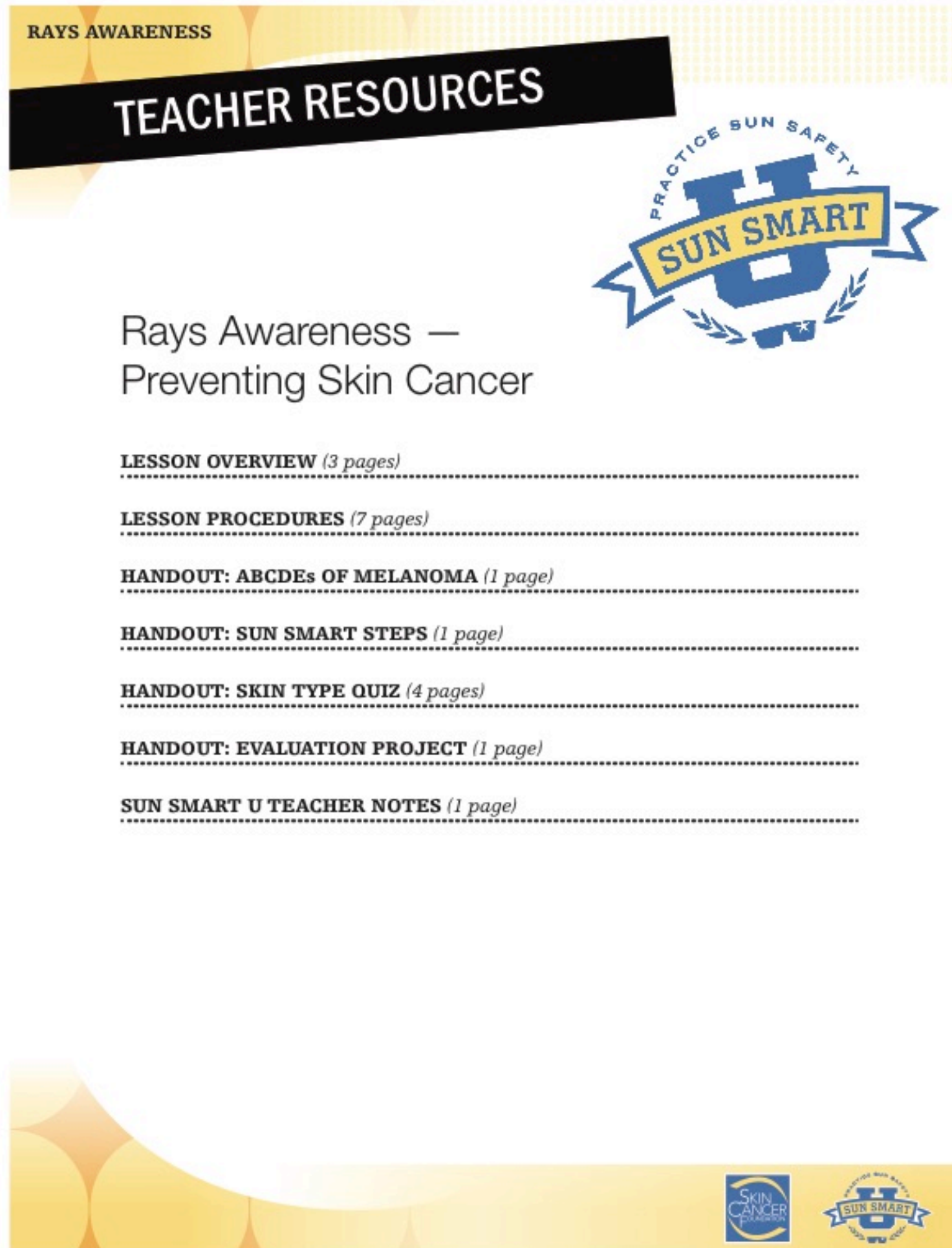

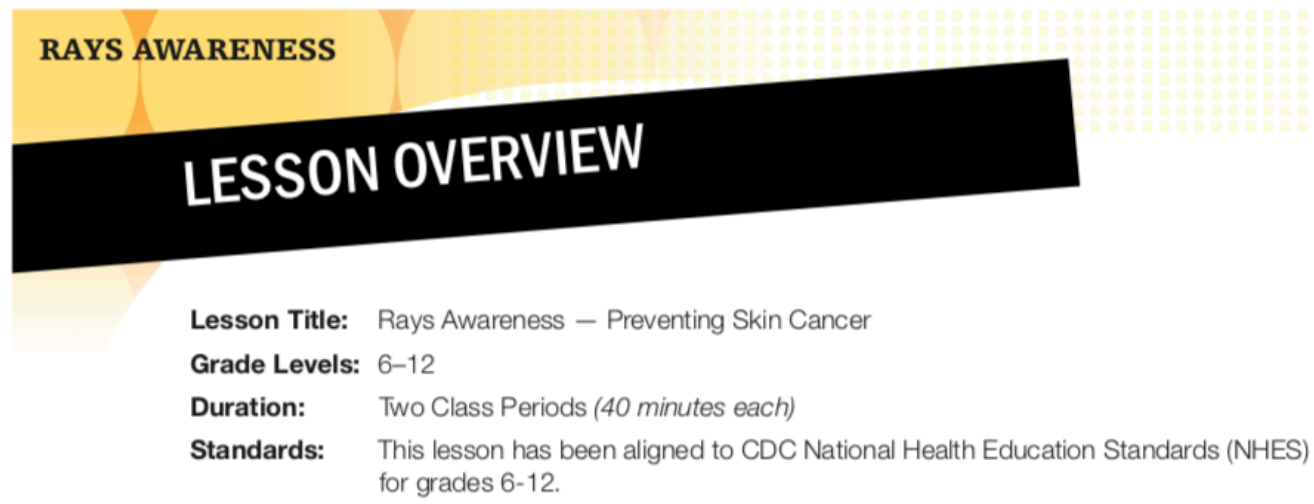

In this lesson, students learn about ultraviolet (UV) radiation from the sun and tanning beds, how it affects their skin, and why protecting themselves from both forms of UV exposure is critical to preventing skin cancer. Two types of UV rays reach Earth's surface: ultraviolet A (UVA) and ultraviolet B (UVB). UVA rays are longer and primarily cause premature skin aging, while UVB rays are shorter and commonly cause sunburns. Both UVA and UVB rays contribute to skin damage, eye damage, and skin cancers. This lesson teaches students lifelong sun safety habits.

\section{OBJECTIVES}

After finishing this lesson, students will be able to

- understand ultraviolet radiation, including UVA and UVB rays.

- explain how UV radiation affects their skin.

- understand the effects of indoor and outdoor tanning.

- understand the various forms of skin cancer.

- understand how to detect skin cancer.

- protect themselves from UV radiation exposure.

\section{TEACHER BACKGROUND INFORMATION}

The sun gives off more than just visible light. It emits waves of energy known as radiation. Ultraviolet radiation (UV) is the form of radiation with wavelengths shorter than visible light on the electromagnetic spectrum. It is classified into three main categories according to wavelength. UVA has the longest wavelength, UVB a shorter wavelength, and UVC shorter still, but it is mostly absorbed by the ozone layer and does not reach the earth.

With a shorter wavelength than UVA, UVB rays can reach only the skin's epidermis (outer layer). UVB radiation is the main cause of sunburn and one of the main causes of skin cancer. The intensity of UVB rays can vary during the day and across the seasons. Although they can damage and burn the skin yearround, the most intense UVB rays hit the continental United States between April and October from 10 AM to 4 PM.

UVA rays have a longer wavelength and can penetrate into the skin's dermis (inner layer). UVA rays are relatively constant during the day and throughout the year and can travel through clouds and glass. Exposure to UVA rays damages the skin's DNA and causes the skin to darken or tan in an attempt to shield the cells from further radiation damage. This tan, which is never healthy, is a sign that cellular damage, which can lead to skin aging and wrinkling as well as skin cancer, has already taken place. 


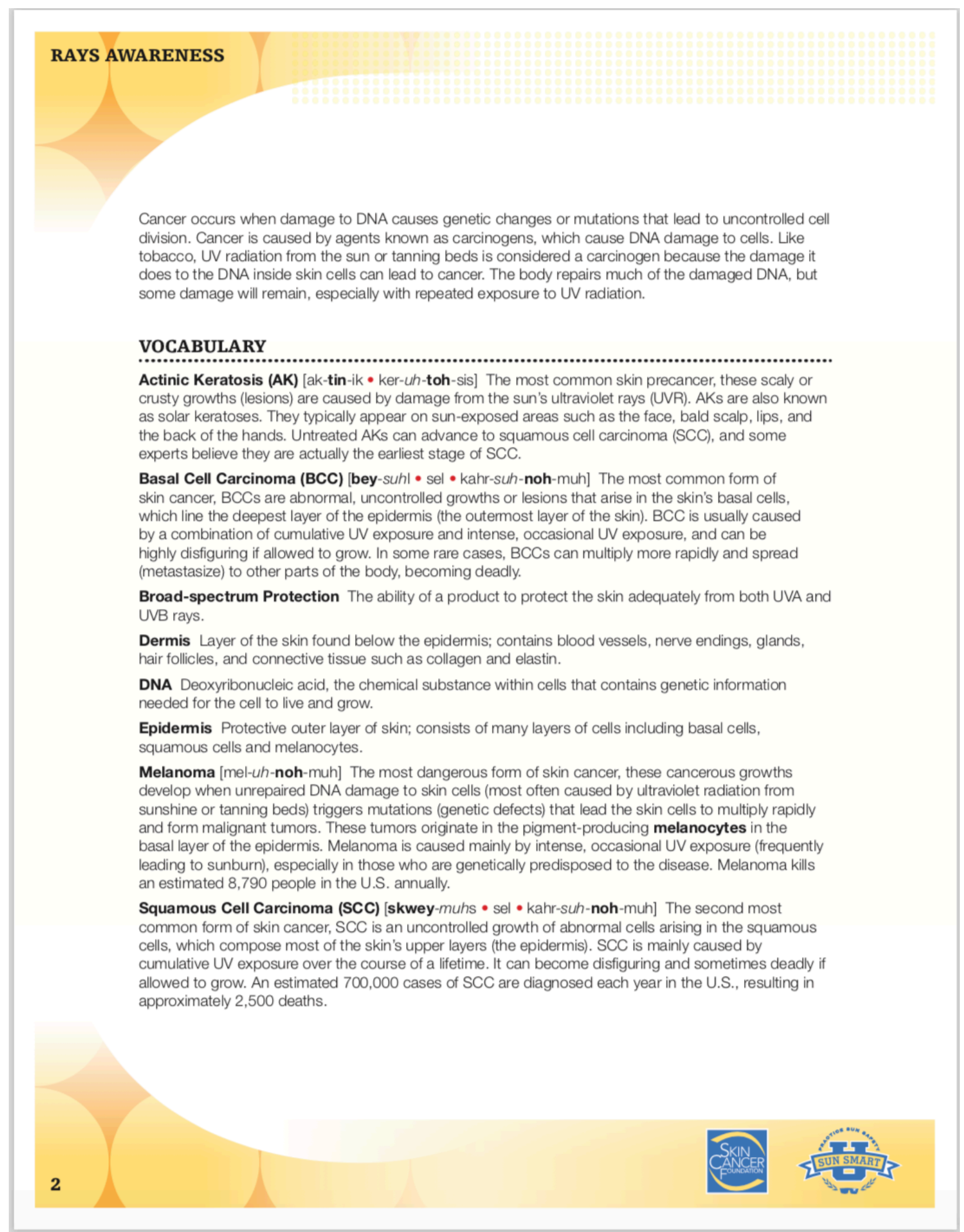




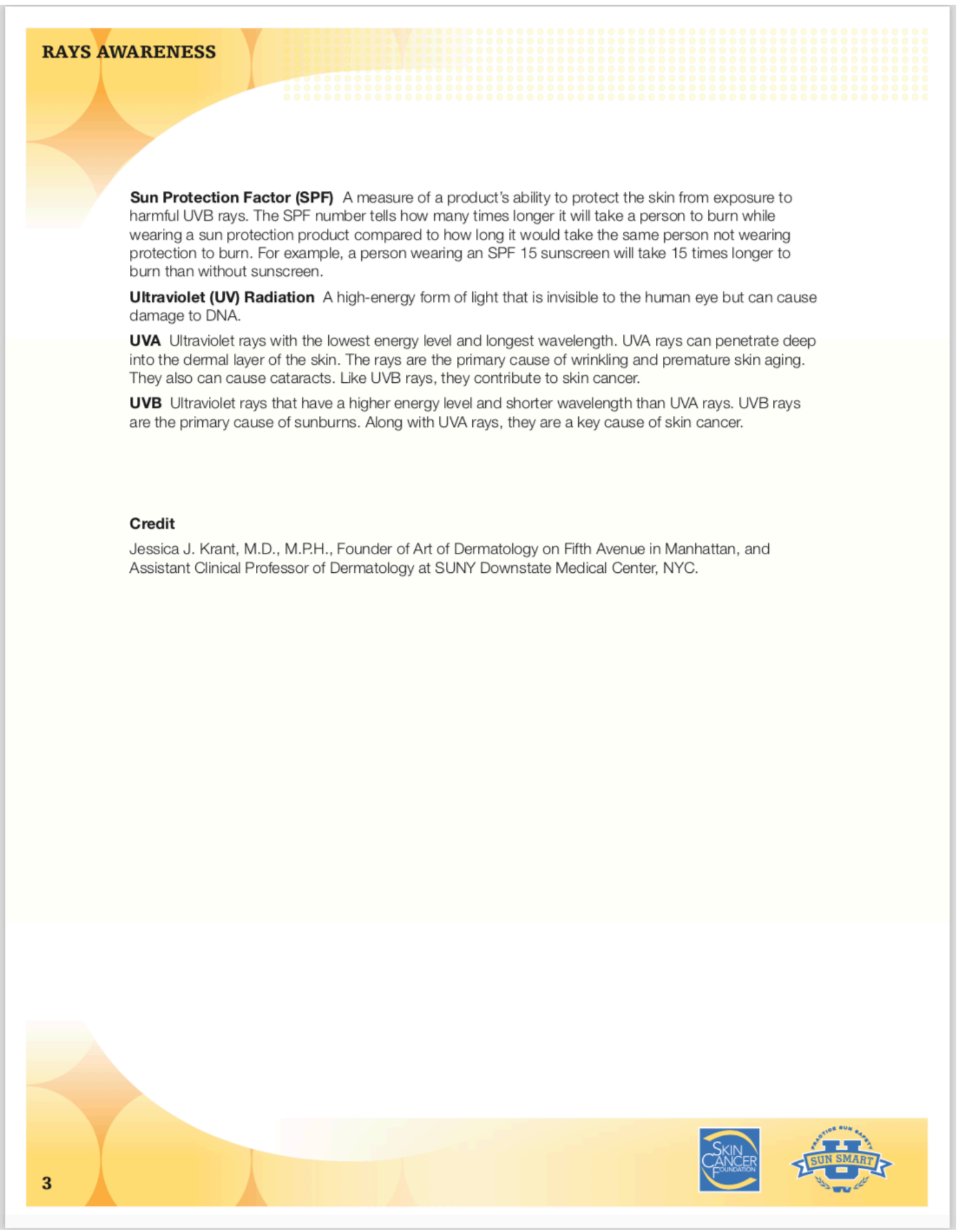




\section{RAYS AWARENESS}

\section{LESSON PROCEDURES}

\section{1) TRUE OR FALSE INTERACTIVE ACTIVITY}

Begin class by quizzing students on skin cancer. Use this activity to gauge your students' knowledge of skin cancer and reveal any misconceptions they may have. Read each statement on the quiz. After each statement, tell students, "Raise your hand if you think this statement is true." Then ask students to raise their hands if they think the statement is false. Note the class majority's response. After the show of hands, read the correct response. Continue to quiz students with each true or false statement.

\section{TRUE OR FALSE?}

1. Statement: Skin cancer is rare. (FALSE)

Fact: 1 in 5 Americans will get skin cancer in their lifetime.

2. Statement: You can die from skin cancer. (TRUE)

Fact: You can die from skin cancer if it spreads to your internal organs. Melanoma is the deadliest form of skin cancer. However, when caught early, about $99 \%$ of all melanomas are curable. That's why it's important to go to a dermatologist to catch skin cancer early-so it doesn't spread and become fatal.

3. Statement: Skin cancer is a disease that affects only old people. (FALSE)

Fact: Melanoma is the second most common form of cancer among 15-29 year olds.

4. Statement: People with dark skin can get skin cancer. (TRUE)

Fact: No one is immune from the dangers of the sun. In fact, skin cancer is rarer but more deadly among darker skinned people.

5. Statement: Experiencing five or more sunburns can double your risk of developing skin cancer. (TRUE)

Fact: Experiencing just one bad sunburn as a child or teen doubles your risk of getting melanoma. Five sunburns by any age also doubles your chances of developing melanoma.

6. Statement: Two forms of ultraviolet radiation - UVA and UVB - reach the earth and can cause skin cancer. (TRUE)

Fact: UVA penetrates the skin even more deeply than UVB and damages the inner layer of skin. These rays play a major role in skin aging and wrinkling, as well as skin cancer. UVB is a shorter wave that damages the skin's top epidermal layers. UVB rays are chief causes of skin reddening and sunburn and play a key role in the development of skin cancer, as well as a role in skin aging.

7. Statement: Though a sunburn is a sign of sun damage, a tan is a sign of health. (FALSE) Fact: Both sunburns and suntans are signs that your skin cells have been damaged by the sun. There is no such thing as a "healthy tan." Both sun tans and bums can lead to skin cancer.

8. Statement: Sunshine clears up acne. (FALSE)

Fact: Sun exposure appears to help acne at first, but in the end it can aggravate it and make it worse. If you use acne medication, be sure to apply sunscreen, wear protective clothing, and seek the shade. Many acne medications make your skin more likely to burn in the sun, which makes you more susceptible to sun damage. 


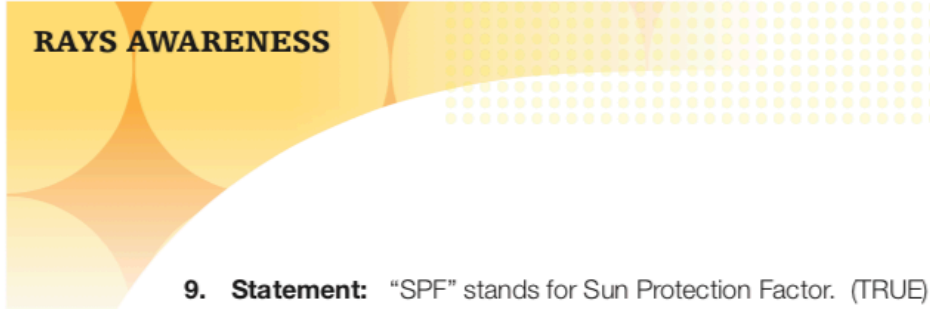

9. Statement: "SPF" stands for Sun Protection Factor. (TRUE)

Fact: SPF indicates how long it will take for UVB rays to redden skin when using a sunscreen, compared to how long skin would take to redden without the product. For instance, someone using a sunscreen with an SPF of 15 will take 15 times longer to redden than they would without the sunscreen. An SPF 15 sunscreen screens approximately 93 percent of the sun's UVB rays; SPF 30 protects against 97 percent.

10. Statement: Tanning beds are safer for you than sunlight. (FALSE)

Fact: There is no such thing as a safe tan, especially in tanning beds. People using tanning beds may receive as much as 12 times the annual dose of harmful UVA radiation compared to the dose they receive from sun exposure. Using a tanning bed increases your risk of melanoma by $74 \%$.

\section{2) VIDEO SEGMENT \# 1 - REAL STORY (CHELSEA)}

Tell students that you want to show them a real story about someone just out of college who has skin cancer. Project the video on a screen or via your interactive whiteboard.

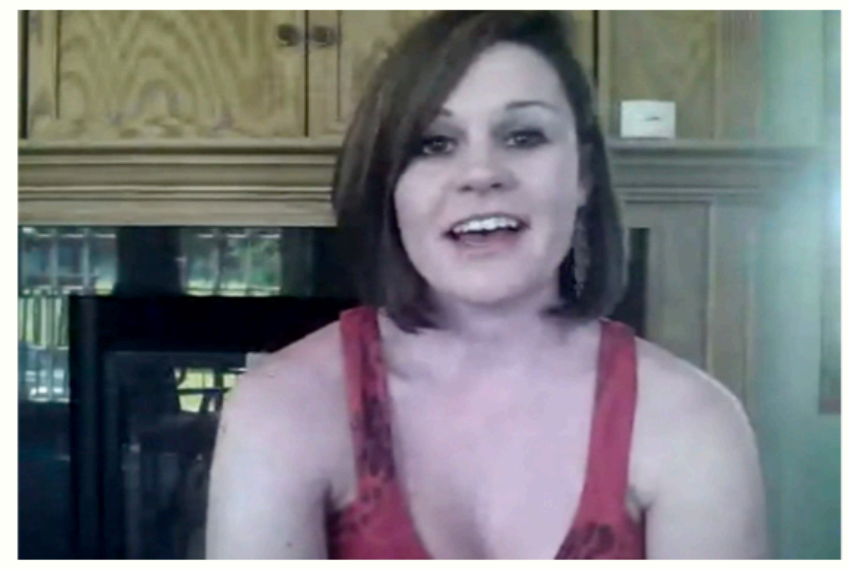

\section{3) WHAT TO LOOK FOR...}

Ask students how many are surprised that this could happen to someone so young. Mention to students that they've learned some facts about skin cancer in this lesson so far and have also heard a personal story from someone who is battling melanoma, the deadliest form of skin cancer. Now tell them that you will show them how to detect various forms of skin cancer.

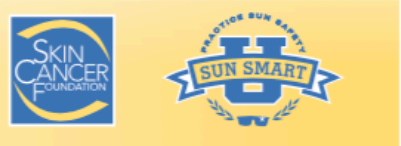




\section{RAYS AWARENESS \\ THE ABCDES OF MELANOMA}

Moles, brown spots and growths on the skin are usually harmless - but not always. Anyone who has more than 100 moles or one or more unusual looking or atypical moles is at greater risk for melanoma. That's why it's so important to get to know your skin very well and to recognize any changes in the moles on your body. Look for the ABCDE signs of melanoma, and if you see one or more, make an appointment with a dermatologist immediately.

The pictures below show normal moles and melanomas.

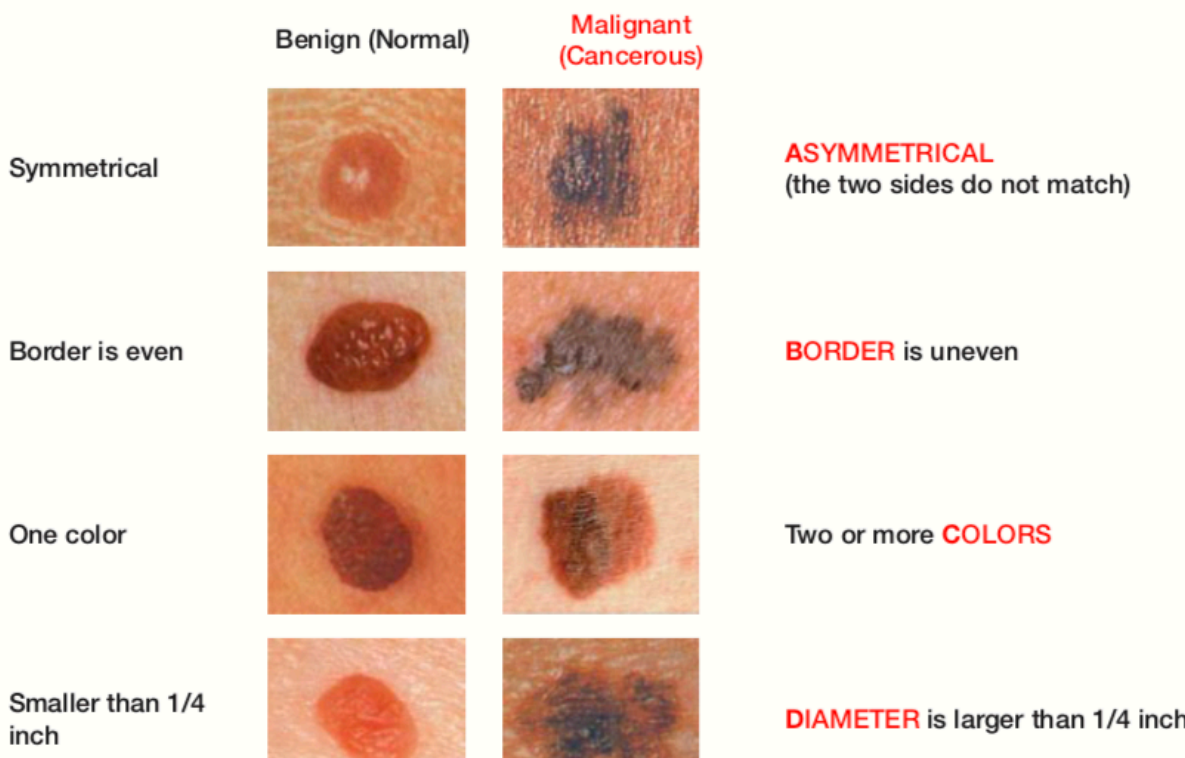

inch
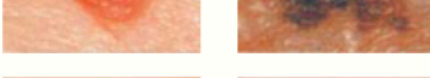

Ordinary mole

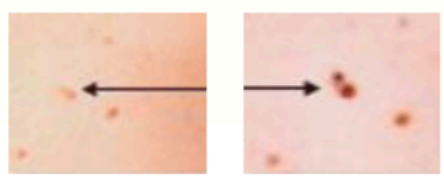

EVOLVING or changing in size, shape, color, or another trait

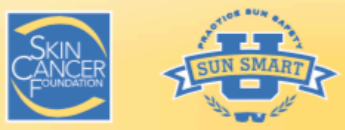




\section{RAYS AWARENESS \\ THE UGLY DUCKLING SIGNS}

Tell students that while the ABCDE rule helps detect many melanomas; some melanomas do not exhibit the ABCDE features. This method is based on the concept that these melanomas look different - they are "ugly ducklings" - compared to other moles nearby. "Normal" moles resemble each other, like siblings, while a potential melanoma looks or feels different than the other moles. Melanomas, unlike normal moles, also tend to keep growing and changing.

These three different scenarios depict "ugly duckling" moles that should prompt suspicion. Squares A, B, and $\mathrm{C}$ each represent a body area such as the back.

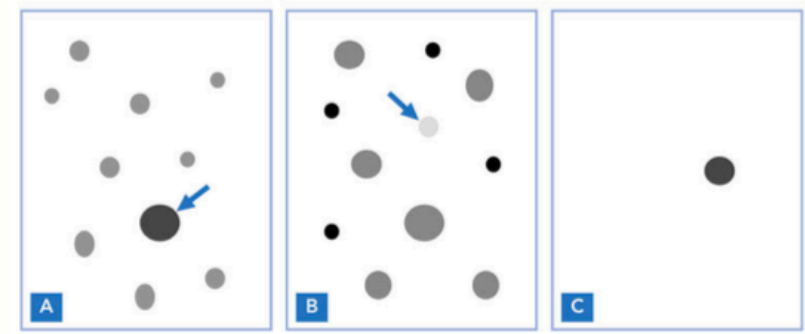

Figure 1. Three Examples of an Ugly Duckling

- In A, there is a dominant mole pattern with slight variation in size. The "ugly duckling" is clearly darker and larger than all other moles.

- In B, there are two main patterns, one of larger moles and the other of smaller, darker moles. The "ugly duckling" is small but lacks pigmentation/color.

- In $\mathbf{C}$, there is only one lesion on the back. If this lesion is changing, causing a problem, or deemed unusual or atypical, see a dermatologist and have this "ugly duckling" examined.

An approach combining the ABCDEs and the "Ugly Duckling" technique should improve the chances of early detection of all types of melanoma and help save lives.

\section{OTHER FORMS OF SKIN CANCER AND PRECANCER}

More than 3.5 million new skin cancers in over two million people are diagnosed every year in the U.S. If caught early, almost all are curable.

\section{BASAL CELL CARCINOMA}

Basal Cell Carcinoma (BCC) is the most common form of skin cancer. It can be a raised, translucent, pearly nodule, a scar-like area that is white, yellow or waxy, a reddish patch or irritated area, an open sore that bleeds, oozes or crusts and doesn't heal, or a pink growth with a slightly elevated border and a crusted indentation in the center. It is usually caused by both cumulative and intermittent, intense sun exposure or tanning machine exposure. 


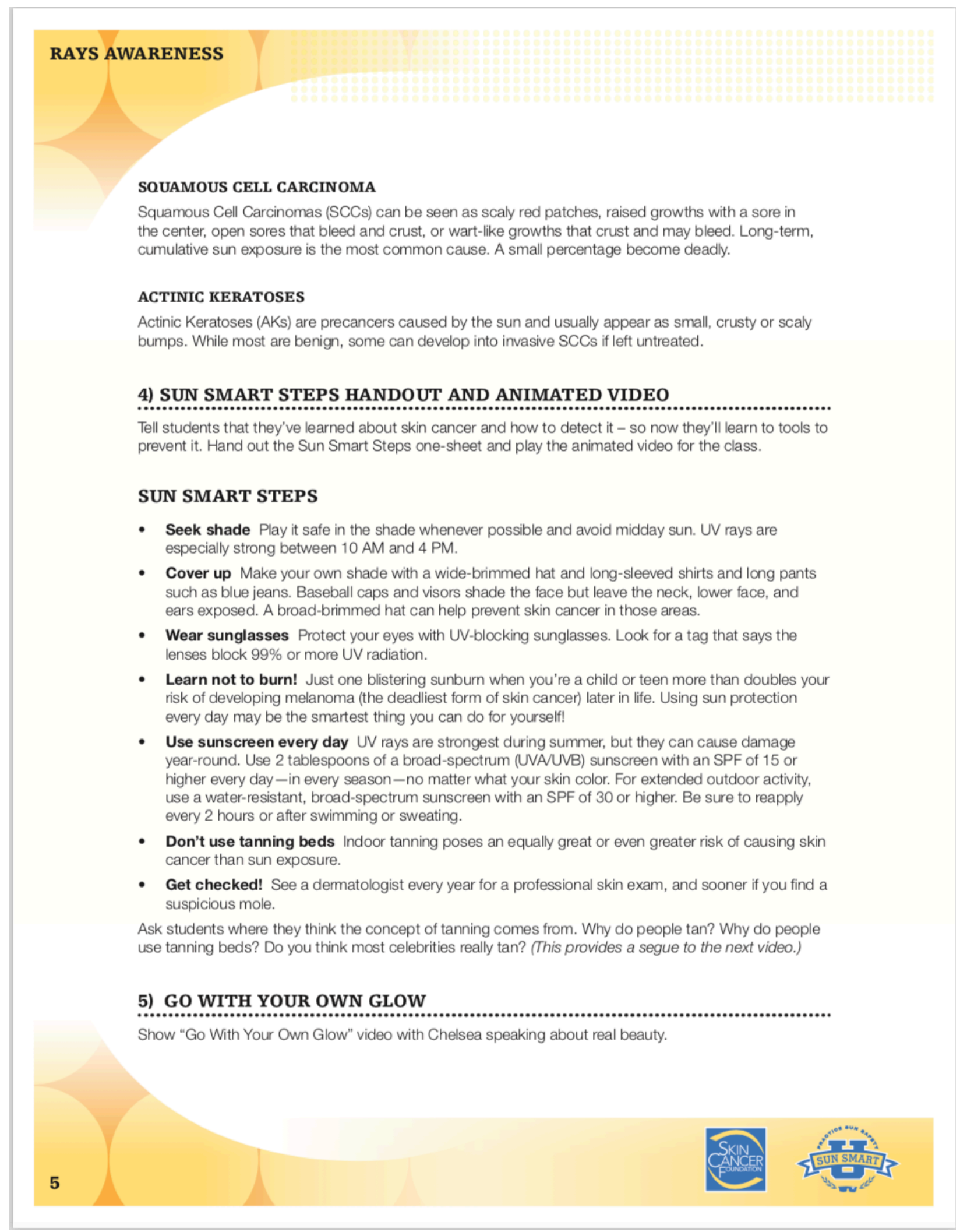




\section{RAYS AWARENESS \\ 6) EVALUATION PROJECT}

Hand out the Skin Type Quiz to each student for them to fill out independently. This will help each student be able to understand their own skin and how the information they've leamed in the lesson can be applied to his/her habits and sun safety procedures.

Who's your Celebrity Skin Twin? After students fill out the Skin Type Quiz, project images of celebrities according to skin type, so the students can see who they resemble. Reveal one skin type at a time with one female celeb and one male celeb per skin type.

Ask the students to write a paragraph or two. "Now that you know your own skin type, how are you going to protect your skin?"

\section{EVALUATION RUBRIC}

How well did your students comprehend the lesson and understand their own skin type in relation to the Sun Smart Steps?

Use the rubric below to rate each student's essay and class participation on a scale of 1 to 10 .

Maximum score is 100. Minimum score is 5.

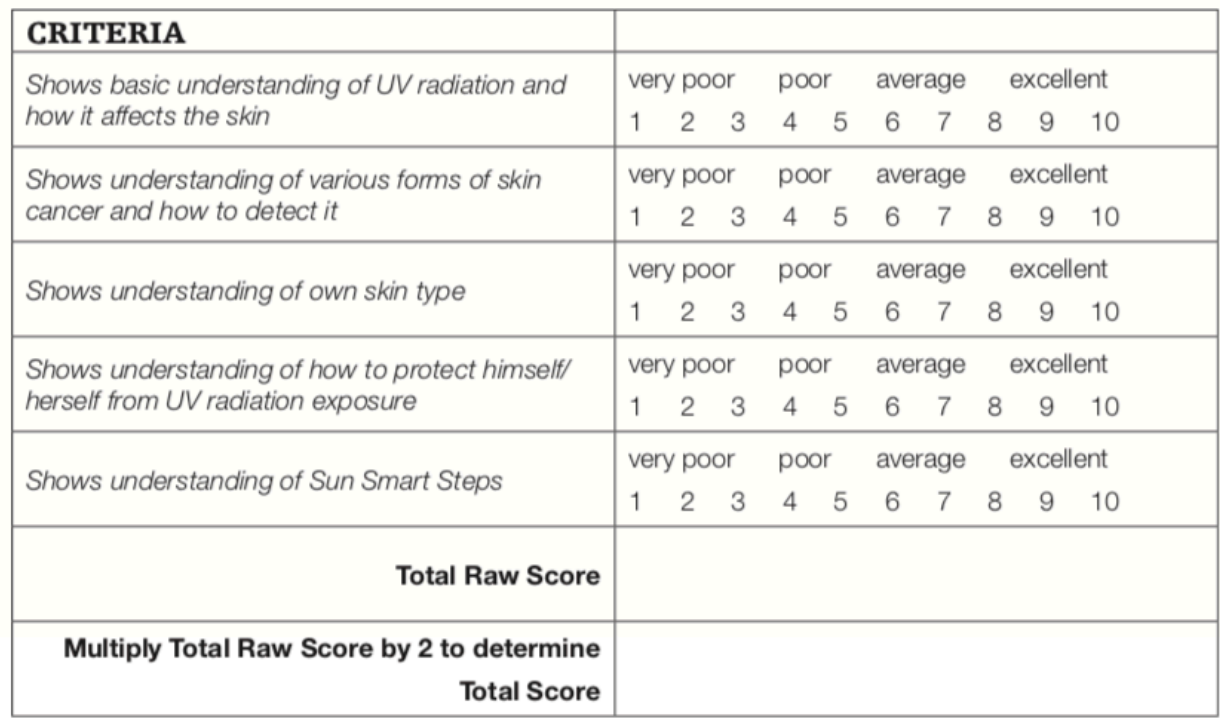

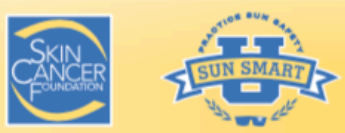




\section{RAYS AWARENESS}

7) LIVE SMART -

VIDEO SEGMENT \#3 REAL STORY CONCLUSION (CHELSEA)

Project final video segment of Chelsea for students to view.

8) TAKE-HOME ACTIVITY: SKIN TYPE QUIZ \& SUN SMART STEPS (not to be graded)

Give students the Skin Type Quiz to bring home to their family members to take. Also, give student the Sun Smart Steps handout to bring home to their families, so they will know how to protect themselves from the sun.

Sun Smart U Interactive Whiteboard elements have been developed and provided as

a generous gift from Saferock Education.

www.saferockeducation.com

1.877.753.7300

info@saferock.com
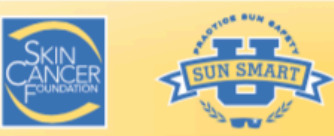


\section{RAYS AWARENESS \\ THE ABCDES OF MELANOMA}

Moles, brown spots and growths on the skin are usually harmless - but not always. Anyone who has more than 100 moles or one or more unusual looking or atypical moles is at greater risk for melanoma. That's why it's so important to get to know your skin very well and to recognize any changes in the moles on your body. Look for the ABCDE signs of melanoma, and if you see one or more, make an appointment with a dermatologist immediately.

The pictures below show normal moles and melanomas.

Symmetrical

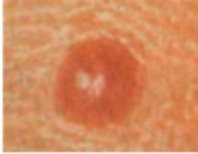

Border is even
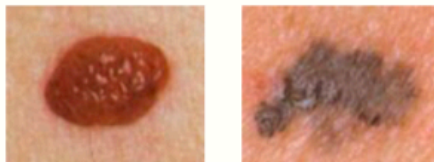

One color
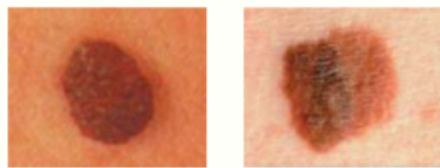

Smaller than $1 / 4$

inch
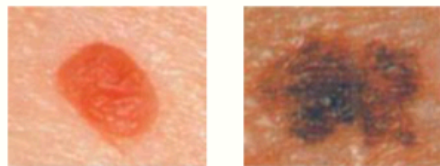

Ordinary mole
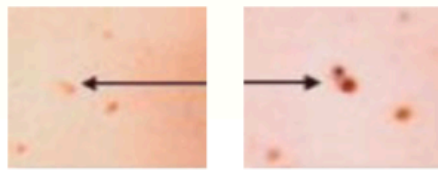

BORDER is uneven

Two or more COLORS

ASYMMETRICAL

(the two sides do not match)

DIAMETER is larger than $1 / 4$ inch

EVOLVING or changing in size, shape, color, or another trait
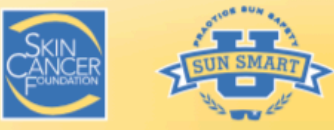


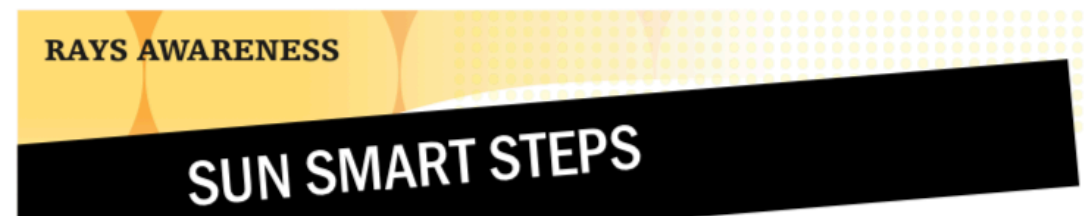

- Seek shade Play it safe in the shade whenever possible and avoid midday sun. UV rays are especially strong between 10 AM and 4 PM.

- Cover up Make your own shade with a wide-brimmed hat and long-sleeved shirts and long pants such as blue jeans. Baseball caps and visors shade the face but leave the neck, lower face, and ears exposed. A broad-brimmed hat can help prevent skin cancer in those areas.

- Wear sunglasses Protect your eyes with UV-blocking sunglasses. Look for a tag that says the lenses block $99 \%$ or more UV radiation.

- Learn not to burn! Just one blistering sunburn when you're a child or teen more than doubles your risk of developing melanoma (the deadliest form of skin cancer) later in life. Using sun protection every day may be the smartest thing you can do for yourself!

- Use sunscreen every day UV rays are strongest during summer, but they can cause damage year-round. Use 2 tablespoons of a broad-spectrum (UVA/UVB) sunscreen with an SPF of 15 or higher every day-in every season-no matter what your skin color. For extended outdoor activity, use a water-resistant, broad-spectrum sunscreen with an SPF of 30 or higher. Be sure to reapply every 2 hours or after swimming or sweating.

- Don't use tanning beds Indoor tanning poses an equally great or even greater risk of causing skin cancer than sun exposure.

- Get checked! See a dermatologist every year for a professional skin exam, and sooner if you find a suspicious mole. 


\section{RAYS AWARENESS}

\section{SKINTYPE QUIZ}

Different skin types react differently to the sun and have different levels of skin cancer risk. Your skin type can be identified by looking at your genetic disposition and your typical reaction to sun exposure. Thomas Fitzpatrick, MD, of Harvard Medical School, developed a simple quiz to help people identify their skin type and the associated skin cancer risks. Knowing your skin type can help you know how to best protect yourself.

Please circle your answer to each of the following questions. Tally your scores for genetic disposition and for reaction to sun exposure. Then, combine those scores to calculate your Skin Type Score and your Fitzpatrick Skin Type.

\section{PART I: GENETIC DISPOSITION}

(circle your answer)

\begin{tabular}{|c|c|c|}
\hline \multirow{5}{*}{ Your eye color is: } & Light blue, light gray, or light green & 0 \\
\hline & Blue, gray, or green & 1 \\
\hline & Hazel or light brown & 2 \\
\hline & Dark brown & 3 \\
\hline & Brownish black & 4 \\
\hline \multirow{5}{*}{ Your natural hair color is: } & Red or light blonde & 0 \\
\hline & Blonde & 1 \\
\hline & Dark blonde or light brown & 2 \\
\hline & Dark brown & 3 \\
\hline & Black & 4 \\
\hline \multirow{5}{*}{$\begin{array}{l}\text { Your natural skin color (before sun } \\
\text { exposure) is: }\end{array}$} & Ivory white & 0 \\
\hline & Fair or pale & 1 \\
\hline & Fair to beige, with golden undertone & 2 \\
\hline & Olive or light brown & 3 \\
\hline & Dark brown & 4 \\
\hline \multirow{5}{*}{$\begin{array}{l}\text { How many freckles to you have on } \\
\text { unexposed areas of your skin? }\end{array}$} & Many & 0 \\
\hline & Several & 1 \\
\hline & A few & 2 \\
\hline & Very few & 3 \\
\hline & None & 4 \\
\hline
\end{tabular}

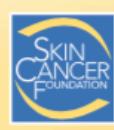




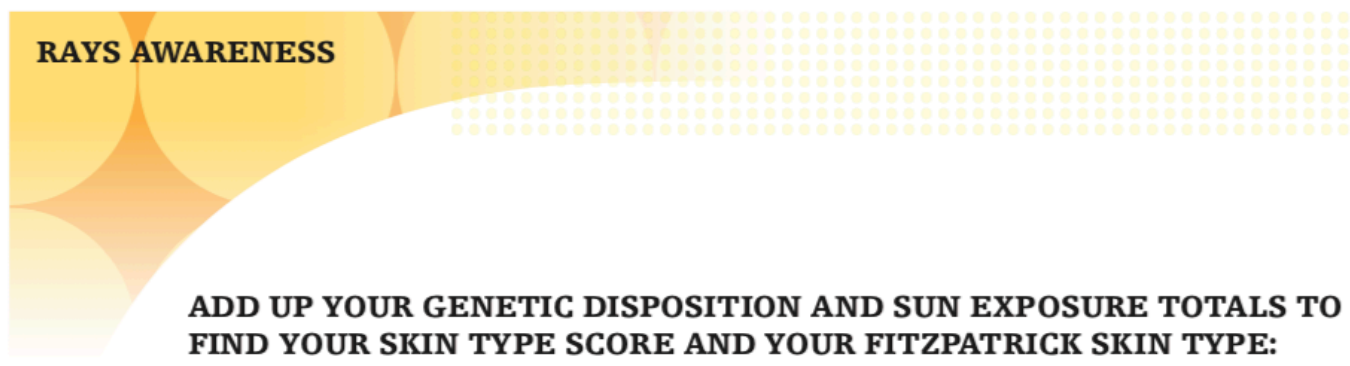

\begin{tabular}{|c|c|c|}
\hline $\begin{array}{l}\text { TOTAL } \\
\text { SCORE }\end{array}$ & & FITZPATRICK SKIN TYPE \\
\hline $0-6$ & I & $\begin{array}{l}\text { Pale, porcelain, ivory-that's you. Your hair tends to be reddish or blonde, and you may } \\
\text { have light eyes. You always burn in the sun, never tan, and you are extremely susceptible } \\
\text { to skin damage as well as cancers like basal cell carcinoma and squamous cell } \\
\text { carcinoma. You are also at very high risk for melanoma, the deadliest type of skin cancer. } \\
\text { Generally, follow the Sun Smart Steps. Use a broad spectrum (UVAUVB) sunscreen } \\
\text { with an SPF of } 30+\text { and clothing with an ultraviolet protection factor (UPF) rating of } 30 \\
\text { or higher. Seek the shade whenever you are out in the sun. Check your skin head-to- } \\
\text { toe each month, paying careful attention to any suspicious growths, and make sure you } \\
\text { have an annual professional skin checkup. }\end{array}$ \\
\hline $7-12$ & II & $\begin{array}{l}\text { You're fair-skinned and may have green, blue, or hazel eyes. You almost always burn } \\
\text { and rarely tan in the sun and are highly susceptible to skin damage, as well as both non- } \\
\text { melanoma and melanoma skin cancers. Generally, follow the Sun Smart Steps. Use a } \\
\text { broad spectrum sunscreen with an SPF of } 30+\text { and clothing with an ultraviolet protection } \\
\text { factor (UPF) rating of } 30 \text { or higher. Seek the shade whenever you are out in the sun. } \\
\text { Check your skin head-to-toe each month, paying careful attention to any suspicious } \\
\text { growths, and make sure you have an annual professional skin checkup. }\end{array}$ \\
\hline $13-18$ & III & $\begin{array}{l}\text { You have light-to-medium skin, which may look peachy or beige. You sometimes burn } \\
\text { and sometimes tan in the sun. You are susceptible to skin damage as well as both non- } \\
\text { melanoma and melanoma skin cancers. Be sure to apply a sunscreen with an SPF of } \\
\text { at least } 15 \text { or higher every day and for extended outdoor activity, use a water-resistant, } \\
\text { broad spectrum sunscreen with an SPF of } 30 \text { or higher. Also wear sun-protective } \\
\text { clothing and seek the shade between } 10 \mathrm{AM} \text { and } 4 \mathrm{PM} \text {, when the sun is strongest. } \\
\text { Follow all other Sun Smart Steps as well. Check your skin head-to-toe each month, } \\
\text { paying careful attention to any suspicious growths, and make sure you have an annual } \\
\text { professional skin checkup. }\end{array}$ \\
\hline 19-24 & IV & $\begin{array}{l}\text { Your skin is golden- or olive-hued. You tend to have dark eyes, tan easily, and are less } \\
\text { likely to burn. Nevertheless, you still need to protect yourself from the sun's harmful } \\
\text { ultraviolet (UV) radiation. Use a broad spectrum sunscreen with an SPF of } 15 \text { or higher } \\
\text { outside, a water-resistant, broad spectrum sunscreen with an SPF of } 30 \text { or higher for } \\
\text { extended outdoor activity, and seek the shade between } 10 \text { AM and } 4 \text { PM. Follow all } \\
\text { other Sun Smart Steps as well. Check your skin head-to-toe each month, paying careful } \\
\text { attention to any suspicious growths, and make sure you have an annual professional skin } \\
\text { checkup. }\end{array}$ \\
\hline
\end{tabular}

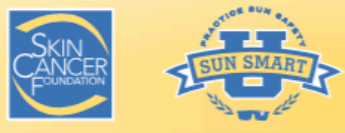




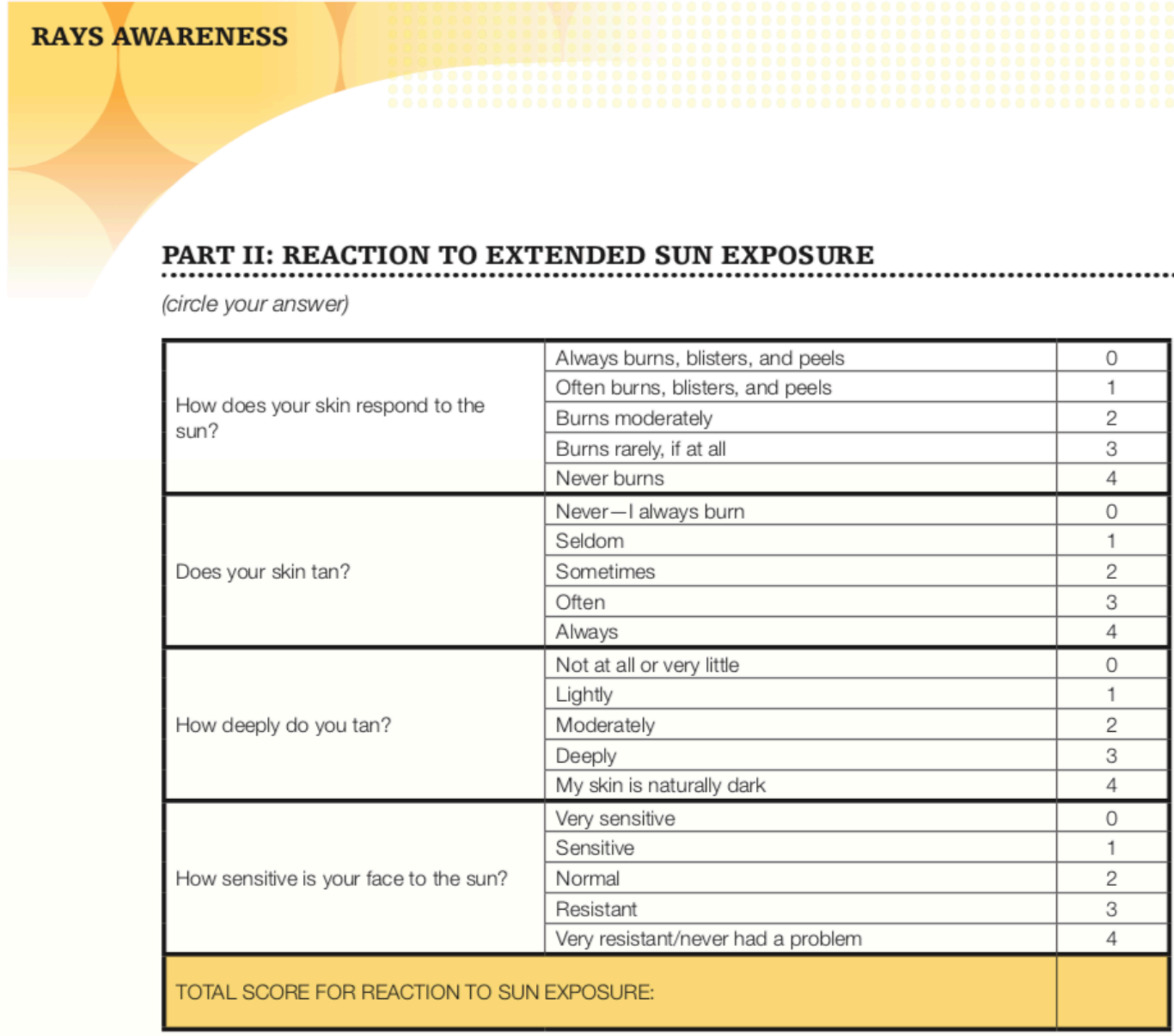

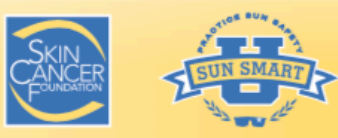




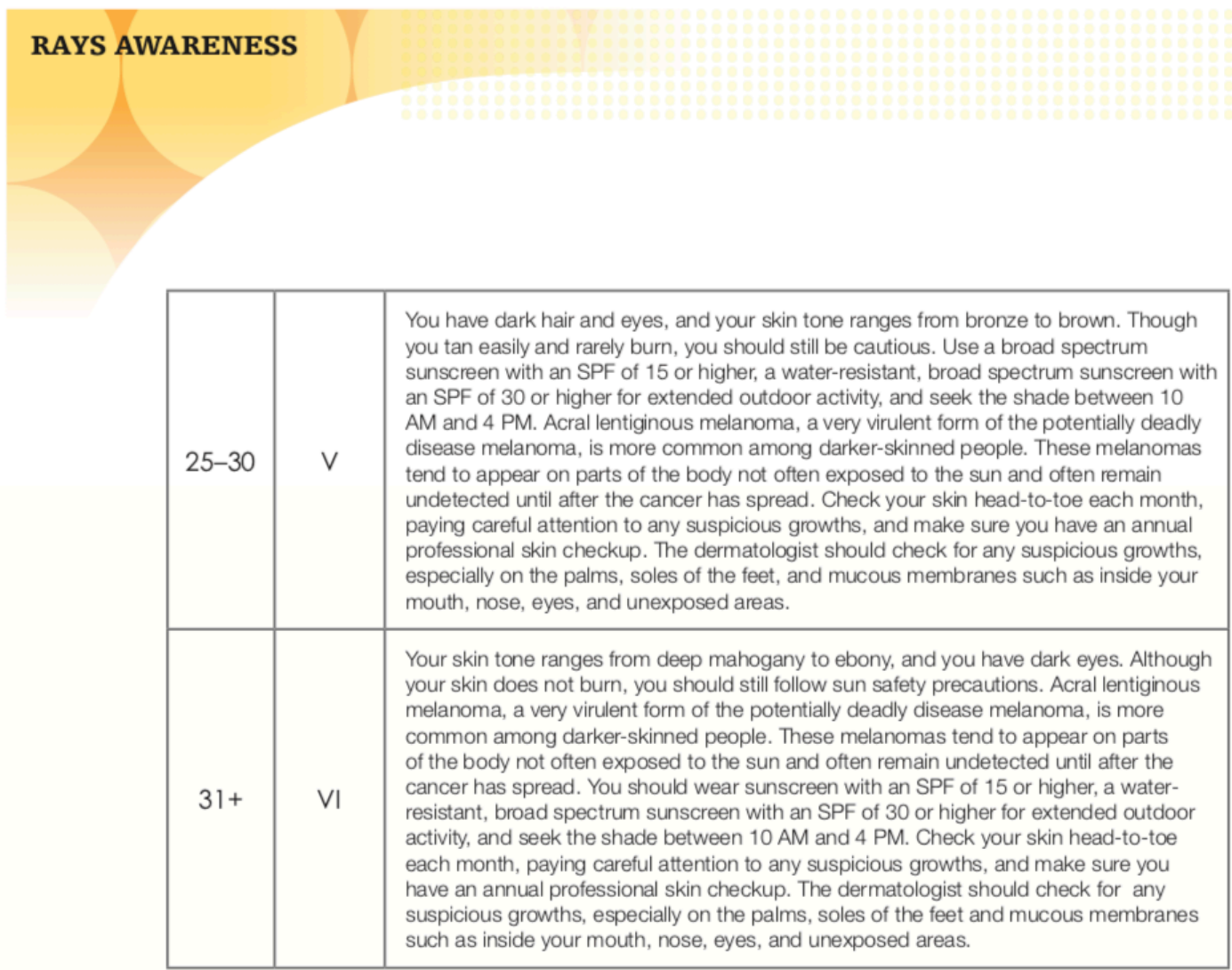

This skin type quiz is for informational purposes only. The content is not intended to be a substitute for professional medical advice, diagnosis, or treatment. Always seek the advice of your physician or other qualified health providers with any questions you may have regarding a medical condition. 


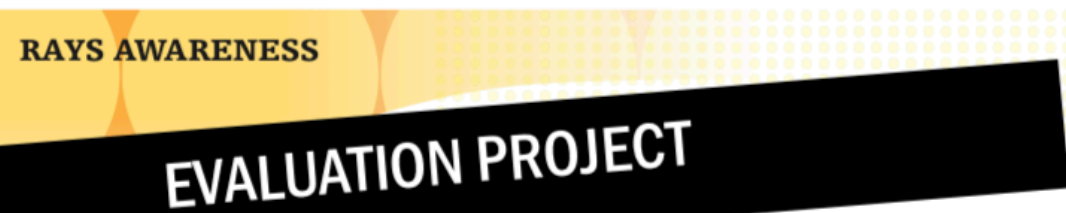

Hand out the Skin Type Quiz to each student for them to fill out independently. This will help each student be able to understand their own skin and how the information they've leamed in the lesson can be applied to his/her habits and sun safety procedures.

Who's your Celebrity Skin Twin? After students fill out the Skin Type Quiz, project images of celebrities according to skin type, so the students can see who they resemble. Reveal one skin type at a time with one female celeb and one male celeb per skin type.

Ask the students to write a paragraph or two. "Now that you know your own skin type, how are you going to protect your skin?"

\section{EVALUATION RUBRIC}

How well did your students comprehend the lesson and understand their own skin type in relation to the Sun Smart Steps?

Use the rubric below to rate each student's essay and class participation on a scale of 1 to 10 .

Maximum score is 100 . Minimum score is 5 .

\begin{tabular}{|c|c|c|c|c|c|c|}
\hline CRITERIA & & & & & & \\
\hline Shows basic understanding of UV radiation and & very poor & poor & average & e & & \\
\hline how it affects the skin & 123 & 45 & 67 & 8 & s & 10 \\
\hline Shows understanding of various forms of skin & very poor & poor & average & ( & & \\
\hline cancer and how to detect it & 123 & 45 & 67 & 8 & s & 10 \\
\hline Showc underctanding fown skin tyno & very poor & poor & average & ( & & \\
\hline snows understanding of own skin type & 123 & 45 & 67 & 8 & s & 10 \\
\hline Shows understanding of how to protect himself/ & very poor & poor & average & & & \\
\hline herself from UV radiation exposure & 123 & 45 & 67 & 8 & s & 10 \\
\hline Chowe undorctonding of $\mathrm{Cun} \mathrm{Cmart} \mathrm{S}$ tene & very poor & poor & average & & & \\
\hline shows understanding or sun smant sleps & 123 & 45 & 67 & 8 & & 10 \\
\hline Total Raw Score & & & & & & \\
\hline Multiply Total Raw Score by 2 to determine & & & & & & \\
\hline Total Score & & & & & & \\
\hline
\end{tabular}

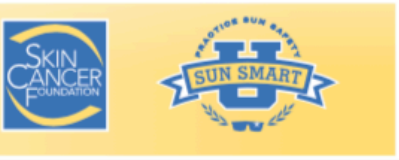



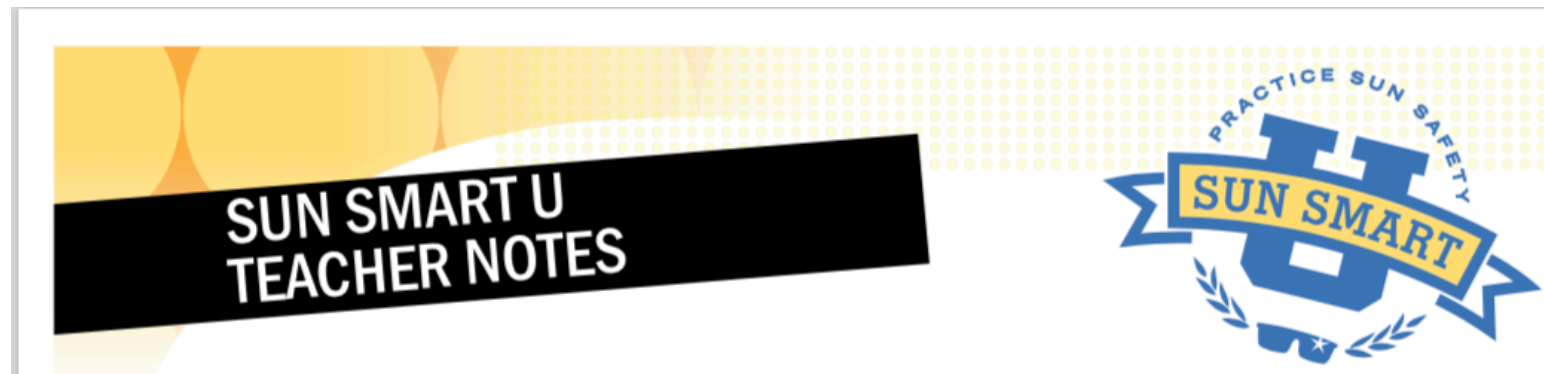

\section{True or False Activity}

Begin class by quizzing students on skin cancer. Use this activity to gauge your students' knowledge of skin cancer and reveal any misconceptions they may have. Read each statement on the quiz. After each statement, tell students, "Raise your hand if you think this statement is true." Then ask students to raise their hands if they think the statement is false. Note the class majority's response. After the show of hands, read the correct response. Continue to quiz students with each true or false statement.

\section{Video Segment \# 1 \\ Real Story:Chelsea}

Tell students that you want to show them a real story about someone just out of college who has skin cancer. Project the video on a screen or via your interactive whiteboard.

\section{The ABCDEs of Melanoma What to look for...}

Ask students how many are surprised that this could happen to someone so young. Mention to students that they've learned some facts about skin cancer in this lesson so far and have also heard a personal story from someone who is battling melanoma, the deadliest form of skin cancer. Now tell them that you will show them how to detect various forms of skin cancer.

\section{The Ugly Duckling Signs}

Tell students that while the ABCDE rule helps detect many melanomas; some melanomas do not exhibit the ABCDE features. This method is based on the concept that these melanomas look different - they are "ugly ducklings" - compared to other moles nearby. "Normal" moles resemble each other, like siblings, while a potential melanoma looks or feels different than the other moles. Melanomas, unlike normal moles, also tend to keep growing and changing.

\section{Sun Smart Steps-Prevention}

Tell students that they've learned about skin cancer and how to detect it - so now they'll learn the tools to prevent it.

\section{Sun Smart Steps-Tanning}

Ask students where they think the concept of tanning comes from. Why do people tan? Do you think most celebrities really tan? Why do people use tanning beds? (This provides a segue to the next video.)

\section{Video Segment \#2}

Show "Go With Your Own Glow" video with Chelsea speaking about real beauty. After viewing the video, hand out the Skin Type Quiz to each student for them to fill out independently. This will help each student be able to understand their own skin and how the information they've learned in the lesson can be applied to his/her habits and sun safety procedures.

\section{Celebrity Skin Type}

Who's your Celebrity Skin Twin? After students fill out the Skin Type Quiz, project images of celebrities according to skin type, so the students can see who they resemble. Reveal one skin type at a time with one female celeb and one male celeb per skin type.

\section{Video Segment \#3}

Project final video segment of Chelsea for students to view. Ask the students to write a paragraph or two. "Now that you know your own skin type, how are you going to protect your skin?" How well did your students comprehend the lesson and understand their own skin type in relation to the Sun Smart Steps? Use the program rubric to rate each student's essay and class participation.

Take-Home Activity:

\section{Skin Type Quiz \& Sun Smart Steps} (not to be graded)

Give students the Skin Type Quiz to bring home to their family members to take. Also, give students the Sun Smart Steps handout to bring home to their families, so they will know how to protect themselves from the sun.
We welcome your feedback.

Please send comments to: sunsmartu@skincancer.org 


\section{Appendix $\mathbf{J}$}

Actual Budget for Project

\begin{tabular}{|c|c|c|}
\hline Budget Categories & Personal Funds & Organizational Contributions \\
\hline ADMINISTRATIVE COSTS & $\$ 0$ & $\begin{array}{l}\text { Hospital staff/employees }(40) \text { for } 40-50 \text { min of education at } \$ 10 / \mathrm{hr} .=\$ 400.00 \\
\text { Fringe benefits (FICA, Workers Compensation, Health Insurance }=\$ 178.73 \\
\text { *No classroom teachers used - intervention performed by student }\end{array}$ \\
\hline \multicolumn{3}{|c|}{$\begin{array}{l}\text { Administrative Justification: No classroom teachers used; student implemented all classroom teaching sessions. Forty staff/healthcare professionals paid for } 40 \text { - } \\
50 \text { minutes of education each ( } 1 \text { sessions of } 40-50 \text { minutes). According to the salary provided by the institution, there have been FICA ( } 7.65 \%) \text {, Worker's } \\
\text { Compensation }(0.54 \%) \text {, and Health Insurance }(30.00 \%) \text { calculated into hourly wages, as noted above. }\end{array}$} \\
\hline MARKETING & $\$ 25.00$ & $\$ 0$ \\
\hline \multicolumn{3}{|c|}{$\begin{array}{l}\text { Marketing Justification: No marketing posters printed to place throughout facility due to limitations of IRB and proposed project timeline. Also, printing for the } \\
\text { curriculum to develop the notebook that will be used to train staff and leave with the Director of Education for further classes. }\end{array}$} \\
\hline EDUCATIONAL MATERIALS/ INCENTIVES & $\$ 0$ & $\$ 0$ \\
\hline \multicolumn{3}{|c|}{$\begin{array}{l}\text { Educational Materials/Incentives Justification: Educational handouts for staff teaching will be provided by SunSmart U at no cost from the Skin Cancer } \\
\text { Foundation. Paper and notebook with pre-developed curriculum will be provided in kind. }\end{array}$} \\
\hline HOSPITALITY (food, room rentals, etc.) & $\$ 250.00$ & $\$ 0$ \\
\hline \multicolumn{3}{|c|}{$\begin{array}{l}\text { Hospitality Justification: The facility will provide the classroom space for implementing the staff educational intervention. There will be no need for rental } \\
\text { materials. Food was provided by the student for all } 4 \text { sessions included in the 'Lunch and Learn' activity session. }\end{array}$} \\
\hline \multicolumn{3}{|c|}{$\begin{array}{l}\text { Project Supplies Justification: The pre and post tests will be provided to the staff on printed paper that will be provided by the DNP student at no cost to the } \\
\text { facility. Writing materials, projector, video monitor, and television will be provided by the facility with no extra cost for the purposes of the project. }\end{array}$} \\
\hline TRAVEL EXPENSES & $\$ 0$ & $\$ 0$ \\
\hline \multicolumn{3}{|c|}{ Travel Expenses Justification: There will be no travel cost for implementation of the proposed project. The implementation will occur on-site at the facility. } \\
\hline OTHER & $\$ 0$ & $\$ 0$ \\
\hline \multicolumn{3}{|l|}{ Other Justification: No other expenses identified. } \\
\hline TOTALS & $\$ 365.00$ & $\$ 578.73$ \\
\hline
\end{tabular}

